\title{
Morpholino-driven transcriptional blockade of Dkk-1 in experimental osteosarcoma inhibits bone damage and tumor expansion by multiple mechanisms.
}

\section{Simin Pan}

Texas A\&M College of Medicine

Michael Cesarek

Texas A\&M College of Medicine

\section{Carla Godoy}

Texas A\&M College of Medicine

\section{Cynthia Co}

Texas A\&M College of Medicine

\section{Catherine Schindler}

Texas A\&M College of Medicine

Kelbi Padilla

Texas A\&M College of Medicine

\section{Roy Poole}

Texas A\&M College of Veterinary Medicine and Biomedical Sciences

\section{Alan Dabney}

Texas A\&M Department of Statistics

\section{Carl Gregory ( $\nabla$ cgregory@tamu.edu )}

Texas A\&M College of Medicine

\section{Article}

Keywords: Reactive Oxygen Species, Nutritional Stress, Chemotherapeutic Challenge, Apoptotic and Necrotic Mechanisms

Posted Date: July 14th, 2021

DOl: https://doi.org/10.21203/rs.3.rs-677459/v1

License: (9) This work is licensed under a Creative Commons Attribution 4.0 International License. Read Full License 

Nature Cancer. Vers 6 7/1/2021.

\section{Morpholino-driven transcriptional blockade of Dkk-1 in experimental osteosarcoma inhibits bone damage and tumor expansion by multiple mechanisms.}

Simin Pan $^{1}$, Michael Cesarek ${ }^{1}$, Carla Godoy ${ }^{1}$, Cynthia M. Co ${ }^{1}$, Catherine Schindler ${ }^{1}$, Kelbi Padilla ${ }^{1}$, Roy Poole ${ }^{2}$, Alan Dabney ${ }^{3}$, Carl A. Gregory ${ }^{1,4}$.

1. Department of Molecular and Cellular Medicine, Institute for Regenerative Medicine, Texas A\&M Health Science Center, Bryan, TX, 77807, USA.

2. Department of Veterinary Pathobiology, Texas A\&M College of Veterinary Medicine and Biomedical Sciences. College Station, TX, 77843, USA

3. Department of Statistics, Texas A\&M University, College Station, TX, 77843, USA

4. Corresponding author: cgregory@tamu.edu 


\section{Abstract. (204 words)}

Osteosarcoma (OS) is the most common primary bone malignancy. Chemotherapy plays an essential role in OS treatment, potentially doubling 5-year event-free survival if tumor necrosis can be stimulated, but long-term treatment results in detriment to health and quality of life. The canonical Wnt inhibitor Dickkopf-1 (Dkk-1) enhances OS survival in part through upregulation of aldehyde-dehydrogenase-1A1 (ALDH1A1) which neutralizes reactive oxygen species from nutritional stress and chemotherapeutic challenge. Dkk-1 also inhibits bone repair, exacerbating formation of osteolytic lesions caused by tumor infiltration. Therefore, targeting the expression of Dkk-1 in OS could reduce tumor burden and increase susceptibility to chemotherapeutics while restoring bone repair.

Herein, we report that inhibiting Dkk-1 transcription by means of a vivo morpholino (DkkMo) reduced the expansion of experimental OS tumors, preserved bone volume and architecture, and stimulated tumor necrosis. This was observed in the presence or absence of doxorubicin (DRB), and as a single agent, inhibition of tumor expansion by DkkMo was equivalent to that achieved by DRB. DkkMo stimulated apoptotic and necrotic mechanisms in tumors and appeared to deplete the tumor stroma. These results indicate that administration of DkkMo with or without chemotherapeutics can substantially improve OS outcome with respect to tumor expansion and osteolytic corruption of bone. 


\section{Introduction.}

Osteosarcoma (OS) is the most common primary bone malignancy. Particularly common in pediatric patients, OS and other primary bone malignancies account for approximately $9 \%$ of pediatric cancer deaths ${ }^{1}$. The current standard of care for treating osteosarcoma is surgery with neoadjuvant and adjuvant chemotherapy ${ }^{2}$. Chemotherapeutic strategies for treating osteosarcoma generally include high-dose methotrexate, doxorubicin (DRB) and cisplatin ${ }^{2}$. These agents undeniably play a positive role in OS treatment, with a reported increase in 5-year event-free survival from approximately $20-40 \%$ (surgery alone) to 50-90\% with successful chemotherapeutic intervention ${ }^{3}$. These survival rates can be stratified into responders with greater than $90 \%$ tumor necrosis after neoadjuvant therapy versus the remainder, who have 5year event-free survival rates of $90 \%$ and $50-60 \%$ respectively ${ }^{3}$. In spite of the clear benefits, long-term use of chemotherapeutic agents results in side effects which can be catastrophic to patients' overall health and quality of life with studies indicating that patients with severe side effects are more likely to be non-adherent to treatment protocols ${ }^{4-6}$. One study indicated that as many as $16 \%$ of patients discontinue adjuvant chemotherapy treatment after persistent side effects ${ }^{5}$. Many patients experience catastrophic pain and immobility as a result of osteolytic bone lesions (OLs), which occur due to excessive bone resorption caused by bone malignancies

7. OLs not only cause significant pain, but also increase risk of fracture and contribute to the vicious cycle between cancer cells, osteoblasts and osteoclasts which provides the ideal environment for tumor cell propagation ${ }^{8}$. Reducing the dependence on chemotherapy and the OL burden would significantly improve the impact of OS treatment strategies.

Several studies report high expression of Dickkopf-Wnt-signaling-pathway-inhibitor-1 (Dkk-1) in various types of cancer, including squamous cell carcinoma, pancreatic cancer, bladder cancer, hepatoblastomas, prostate, breast, multiple myeloma (MM) and OS ${ }^{9-14}$. Our 
previous study found that constitutively high expression of human Dkk-1 in the OS cell line MOSJ-Dkk1 increases tumor growth rate and bone destruction in mouse models when compared to the parental and control lines which manifested tumors primarily as non-osteolytic osteochondral nodules ${ }^{15}$. The observed increase in proliferation and tumorigenicity was found to be due in part to a stress-response modulated by enhanced expression of aldehydedehydrogenase-1A1 (ALDH1A1). This response occurred through constitutive inhibition of canonical Wnt signaling (cWnt) by Dkk-1, driving the balance of Wnt signaling in favor of a non-canonical Wnt pathway (ncWnt) which upregulated ALDH1A1 expression ultimately through activation of Jun kinase (JNK). ALDH1A1 is a known member of the cellular stress response arsenal, neutralizing free radicals from metabolic stressors and chemotherapeutics ${ }^{15}$. The extended family of aldehyde dehydrogenases has also been implicated as a major driver of chemoresistance and survival in cancer stem cells ${ }^{16}$. High levels of Dkk-1 also inhibit cWntmediated differentiation of osteoprogenitors to osteoblasts thereby preventing the repair of OLs in a range of malignant bone diseases (MBD) including OS ${ }^{12,15}$. Therefore, targeting the expression of Dkk-1 in OS tumors could reduce the rate of expansion and survival of tumor cells, increase susceptibility to chemotherapeutics, and restore the capacity of bone to repair itself.

Herein, we demonstrate that through inhibiting Dkk-1 transcription by means of a vivo morpholino (DkkMo), it is possible to (i) reduce the expansion of MOSJ-Dkk1 tumors in vitro and vivo, (ii) preserve bone volume and architecture in vivo, and (iii) stimulate necrosis of the tumor. DkkMo had the capacity to perform these functions in the presence or absence of DRB, and the level of tumor growth inhibition by DkkMo when administered as a single agent was equivalent to that observed by DRB at high dose. In contrast with DRB, the DkkMo morpholino did not cause weight loss in experimental animals. RNA sequencing studies indicated that 
DkkMo stimulated cell death and necrotic mechanisms in tumors, and it also appeared to deplete elements of the tumor stroma.

Collectively, these results indicate that administration of DkkMo in the presence or absence of chemotherapeutic agents has the capacity to substantially improve outcome with respect to OS tumor expansion and osteolytic corruption of bone.

\section{$\underline{\text { Results. }}$}

Blockade of Dkk1 secretion by MOSJ-Dkk1 cells with a vivo morpholino: Previously we demonstrated that constitutive expression of human Dkk-1 by the murine osteochondral sarcoma cell line MOSJ ${ }^{17}$ (MOSJ-Dkk1 cells) resulted in accelerated tumor growth and a significantly more aggressive osteolytic phenotype than parental MOSJ cells or vector control MOSJ (MOSJpLenti) cells when implanted into nude mice ${ }^{15}$. Given the apparent role of Dkk-1 in tumor propagation and bone destruction in several MBDs, we hypothesized that blockade of Dkk-1 could have a dual role in the inhibition of bone destruction and tumor expansion in these diseases. Immunoblockade of Dkk-1 has been successfully achieved in experimental models of MM

18 and OS ${ }^{19}$, but systemic Dkk-1 levels can be substantial ${ }^{10,12}$, requiring large doses of antibody. We therefore hypothesized that blockade of Dkk-1 transcription may represent a more efficient approach in these systems.

To facilitate in vivo delivery and biodistribution of the blocking nucleotide, vivo morpholino (VM) technology was chosen, and a custom VM targeting the 25 nucleotides at the 5' end of the cDNA was custom designed by Gene tools LLC. The VM, hereafter referred to as DkkMo, is complementary to the cDNA encoding first 6 amino acids and the 7 preceding nucleotides (Fig1A). To serve as a control, a similar VM was designed with 5 mismatches 
(scrMo). DkkMo (but not scrMo) dose-dependently reduced secretion of Dkk-1 when added to cultures of MOSJ-Dkk1 cells (Fig1B). In contrast with MOSJ-Dkk1 cells, MOSJ-pLenti cells that do not express high levels of Dkk-1 have a slower rate of proliferation (Fig1C) and are sensitive to confluence and nutritional stressors ${ }^{15}$. MOSJ-Dkk1 cells usually survive for extended durations under these conditions, but as expected, exposure to DkkMo negated this capability causing disruption of monolayers (Fig1D). Loss of the capacity to generate Dkk-1 through the addition of DkkMo also reduced the rate of proliferation of MOSJ-Dkk1 cells (Fig1E\&F). Normalization of Dkk-1 measurements to cell number confirmed that reduced cell counts did not account for the reduced Dkk-1 output (Fig1G).

\section{DkkMo treatment reduces aldehyde dehydrogenase $1 A 1$ production and disrupts stress}

response and anti-apoptotic pathways in vitro: In MOSJ-Dkk1 OS cells, Dkk-1 triggers a stress response pathway by inhibition of cWnt signaling, thereby activating a ncWnt pathway that facilitates JNK/Jun mediated expression of ALDH1A1 ${ }^{15}$. It is known that ALDH1A1 is one of the major aldehyde dehydrogenases responsible for neutralization of reactive oxygen species that occur when cells are subjected to nutritional and chemotherapeutic stress ${ }^{20-23}$, and ALDH1A1 enhances a variety of tumorigenic characteristics in bone cancer cells such as chemoresistance, metastasis and the maintenance of a tumor initiator phenotype ${ }^{20,24-27}$. It was therefore hypothesized that inhibition of Dkk-1 through the action of DkkMo may increase susceptibility to nutritional and chemotherapeutic stressors by reducing expression of ALDH1A1. To test the effect of DkkMo on ALDH1A1 expression, MOSJ-Dkk1 cells were exposed to $5 \mu \mathrm{M}$ DkkMo for 4, 6 and 9 days and ALDH1A1 transcriptional activity was assayed by quantitative RT-PCR (qRTPCR). At each time point, DkkMo reduced ALDH1A1 transcription in MOSJ-Dkk1 cells to levels observed in MOSJ-pLenti cells [Fig2A]. 
To gain broader insight into the potential effects of Dkk1 blockade and reduction in ALDH1A1 activity, MOSJ-Dkk1 cells at logarithmic and confluent phases of growth were exposed to DkkMo for 6 days and mRNA was recovered for high throughput sequencing (HTS). The rationale for these culture conditions was to induce cellular stress through rapid mitosis or nutritional stress respectively. Differentially expressed (DE) sequences (by $>1.5$-fold) between DkkMo treated and DkkScr treated cultures were identified (Supplemental Table 1) and categorized based on gene ontology term enrichment analysis (GOTEA). In both culture conditions, the greatest degree of DE mRNA enrichment occurred in GO-term lists related to stress response, programmed cell death, and response to chemical stimuli (Fig2B\&C). In the case of rapidly dividing cells, GO-terms related to chemotaxis, osteogenic differentiation and immune-chemokines were also represented (Fig2B). Co-expressed, functionally related gene modules were also calculated from the dataset and hub genes with the high highest degree of connectivity within each module were also identified (FigS2). Hub genes were plotted on volcano plots to visualize potential overlap between lists of DE genes (Fig2D\&E). In response to DkkMo, ALDH1A1 was downregulated in both culture conditions, and was identified as a key hub gene, further supporting the close relationship between Dkk-1 and ALDH1A1. Hsd17b4, encoding 17 $\beta$-hydroxysteroid dehydrogenase/D-3-hydroxy acyl-CoA dehydrogenase also met these criteria $^{28}$. In confluent MOSJ-Dkk1 cells only, DkkMo caused downregulation of transcript S1pr2, encoding sphingosine-1-phosphate receptor $2^{29}$. Ingenuity Pathway Analysis (IPA) was performed on DE datasets to identify differentially regulated processes in response to Dkk-1. For both culture conditions, DkkMo treatment downregulated genes involved in tumor cell accumulation (Fig2F\&G). These data indicate that Dkk-1 blockade by DkkMo disrupts 
mitotic and survival pathways involved in the expansion of MOSJ-Dkk1 cells and provides strong additional support for the role of ALDH1A1 in this process.

Dkk-1 Expression Desensitizes MOSJ cells to Doxorubicin: DRB is commonly used alone or in combination for adjuvant treatment of $\mathrm{OS}^{3}$, and chemoresistance to DRB in OS has been attributed in part to elevated ALDH activity ${ }^{20,30}$. This observation is not surprising, given that one of the key mechanisms of DRB is the generation of mitochondrial reactive oxygen species (ROS) which destroys tumor cells but also contributes to a dangerous level of cardiotoxicity ${ }^{31}$. We hypothesized that Dkk-1 expression and associated ALDH1A1 levels could increase resistance to DRB, and the blockade of Dkk-1 by DkkMo could reverse this phenomenon. Identical cultures of rapidly expanding MOSJ-Dkk1 and MOSJ-pLenti cells were subjected to exposure to DRB at doses ranging from $10^{-2}-10^{-8} \mathrm{M}$ over a 10-day duration, and from these growth curves, $\mathrm{IC}_{50}$ values at a range of timepoints were calculated (Fig3A\&C). The $\mathrm{IC}_{50}$ of DRB was consistently higher in MOSJ-Dkk1 cells at all time-points with the greatest reduction in $\mathrm{IC}_{50}$ occurring at day 4 for both cell lines (Fig3A). The day 4 time-point in the cultures corresponded to the initiation of the rapid logarithmic phase of growth (Fig3B, plot) and the difference between the IC 50 in MOSJ-Dkk1 cells versus MOSJ-pLenti cells was greatest at this point (Fig3B, bars). Collectively, these data indicate that Dkk-1 expression increased resistance to DRB in expanding MOSJ cultures, especially during the rapid phase of proliferation. It was therefore hypothesized that Dkk-1 blockade through the action of DkkMo could increase the effectiveness of DRB especially during periods of rapid mitosis. This was tested by exposure of identical cultures of expanding MOSJ-Dkk1 cells to combinations of DRB (at IC50(day4)) and DkkMo (2.5 and $5.0 \mu \mathrm{M})$ for 2, 4, 6, and 9-day periods. In these assays, DRB alone killed approximately half of the cells, but in the presence of $5.0 \mu \mathrm{M}$ DkkMo, this rate increased to 
approximately $100 \%$ at all time-points tested (Fig3D). DkkMo at $5.0 \mu \mathrm{M}$ also possessed an intrinsic capacity to reduce the accumulation of MOSJ-Dkk1 cells to a degree comparable to DRB alone. DkkMo at a dose of $2.5 \mu \mathrm{M}$ exhibited similar activity, but this was confined to day 4 cultures. These data demonstrated that DkkMo could inhibit the expansion of MOSJ-Dkk1 cells and significantly sensitized the cells to DRB.

\section{DkkMo has the capacity to inhibit MOSJ-Dkk1 tumor expansion and induce necrosis in vivo:}

Fluorescently labeled (dsRed) MOSJ-Dkk1 OS were initiated in hindlimbs of immune deficient nude mice followed by administration of DRB (5 mg. $\left.\mathrm{kg}^{-1}\right)$, DKKMo $\left(12.5 \mathrm{mg} \cdot \mathrm{kg}^{-1}\right)$, or combination, by intraperitoneal injection every $48 \mathrm{hr}$. The DRB dose was selected as the highest level that could be administered without significantly affecting survival (FigS1A). Since scrMo had no discernible effect on MOSJ-Dkk1 cells in vitro, and the systemic effects of the backbone morpholino are negligible ${ }^{32,33}$, control (no treatment) mice received saline only. Tumor expansion was followed in live animals by measurement of fluorescent intensity after each administration. Fluorescence intensity values were normalized to the tumor signal at time $=0$ and plotted against time for each animal (Fig4A, B). In each case, the slopes (representing the tumor growth rate) were compared using a mixed-model for repeated measures (MMRM) approach (Fig4B, FigS1B). Fluorescence measurements indicated that combination therapy and DkkMo alone reduced the rate of tumor expansion as compared to the untreated control group but co-administration of DRB did not appear to improve the effect of DkkMo alone. DRB treatment alone reduced the average rate of tumor expansion to a degree equivalent to what was observed for DkkMo alone. The lack of observed synergy or additive contribution between DRB and DkkMo could result from DRB stimulating ALDH1A1 through an alternate pathway, but DRB alone did not increase ALDH1A1 transcription even though endogenous murine Dkk-1 was 
slightly upregulated (FigS1C-E). It is also noteworthy to add that DkkMo administration did not affect the weight of the mice whereas DRB toxicity resulted in significant weight loss (Fig4C). After 2 weeks of treatment, hindlimbs harboring tumors were dissected from euthanized mice and stained with Lugol's iodine contrast agent. On detailed inspection of the axial reconstructed scans, radio-dense patches were observed in the tumor masses that were more prevalent in tumors that received DkkMo and combination treatment (Fig4D, arrowed). A convolutional neural network algorithm (CNNA) was trained to segment the tumor and the radio-dense patches on the axial reconstructions with $81.87 \%$ accuracy (97\% on the validation dataset) (Fig 4D, FigS3). When the CNNA was employed to measure the volume of radio-dense structures in all tumor specimens and normalize this to total tumor volume, there was a significant increase in tumors that were treated with DkkMo or combination (Fig4E, FigS3). MOSJ-Dkk1 tumors consist of a pleomorphic mass of tightly packed undifferentiated fibroblastoid cells that resemble high-grade undifferentiated OS tumors associated with poor prognosis (FigS4A-C). In contrast, histological analysis of the radio-dense structures indicated acellular foci (Fig4F, FigS4D-G, asterisks) that had in most cases become infiltrated with collagenous matrix which stained a characteristic blue with Masson's trichrome stain (FigS5). Cells adjacent to the acellular foci often exhibited clear signs of pyknosis and nuclear fragmentation characteristic of necrosis (FigS5C). Collectively, the data indicated that the radio-opaque structured were collagenized necrotic foci, and that DkkMo not only slowed tumor expansion, but also had the capacity to induce tumor necrosis in vivo.

DkkMo has the capacity to reduce bone destruction in vivo: Several studies have demonstrated that Dkk-1 has the capacity to prevent bone repair in osteolytic lesions, thereby accelerating bone destruction and facilitating tumor expansion ${ }^{10,12}$. Parental MOSJ-cells generate highly 
differentiated osteochondral tumors with limited bone involvement, but MOSJ-Dkk1 cells generate primitive, aggressive and highly osteolytic tumors in mice ${ }^{15}$. To examine whether DkkMo treatment could reduce the osteolytic effects of MOSJ-Dkk1 cells, bones in the tumorbearing hindlimbs of mice were scanned by $\mu \mathrm{CT}$ and volumetrically analyzed. Qualitative inspection of tibias and fibulas indicated that MOSJ-Dkk1 tumors caused significant destruction. To explore this more closely, computational superimposition of scanned images of malformed bones onto unaffected contralateral control scans highlighted where, and to what extent, the surface topology of the bone had deviated from the wild-type form. By measuring these deviations for each voxel, a profile could be generated illustrating the frequency of voxels that had deviated outwards or inwards from the plane of the healthy bone surface. A healthy bone measurement generates a narrow profile, indicating that few voxels deviated from the plane of the contralateral control bone, and if so, by a small degree (Fig 5A, above). A deformed bone would generate a broader distribution, indicating a surface topology consisting of many voxels above and below the plane of the surface of the contralateral control bone (Fig 5A, below). When applied to tibial specimens, healthy bones generated the expected narrow profile whereas untreated MOSJ-Dkk1 bearing tibias demonstrated a high degree of deformation, generating broad profiles with the same true for DRB treated specimens (Fig5B). In contrast, tibias from tumor-bearing mice that received DkkMo or combination therapy generated narrower profiles, indicating less deformation (Fig5B). Statistical analysis of the plots demonstrated that when compared to the no treatment group, the DkkMo plots differed in terms of $\mathrm{x}$-axis positioning $(\mathrm{p}=$ 0.018 with DkkMo, distributions significantly closer to zero) and breadth of the distribution $(\mathrm{p}=$ 0.05 with DkkMo distributions significantly narrower), indicating that DkkMo prevented bone deformation. The same trend was evident for the combination group, but $\mathrm{p}$-values were larger ( $\mathrm{p}$ 
$=0.079$ for $\mathrm{x}$-axis, $\mathrm{p}=0.192$ for distribution), suggesting that $\mathrm{DRB}$ might hinder the osteoprotective action of DkkMo. MicroCT measurement of the tibias and fibulas further confirmed that DkkMo reduced a tumor-associated drop in bone mineral density (Fig5C, left) and absorptive destruction of the bone tissue (Fig5C, right). Osteolytic activity of MOSJ-Dkk1 cells was widespread in untreated and DRB-treated specimens, but this was less apparent in specimens that received DkkMo or combination (Fig5D).

\section{Transcriptomic sequencing of MOSJ-Dkk1 tumors indicates that DkkMo modulates} proliferative, survival and immunological processes in vivo: To gain insight into the antitumorigenic mechanism of action of DkkMo on MOSJ-Dkk1 tumors in vivo, fixed tumors were excised and subjected to HTS. Comparison between untreated (NT) and DkkMo specimens $(n=4)$ and comparison between DRB treated and combination specimens $(n=4)$ were performed. After processing, lists of transcripts with $>1.5$-fold differential expression were generated (Supplemental Table 2). When the lists were subjected to GOTEA, clusters representing regulation of cell stress, cell death and apoptosis were highlighted in both comparisons, further supporting a role for Dkk-1 in the regulation of stress response and survival pathways, and that DkkMo has the capacity to blunt such activities (Fig6A\&B). Unexpectedly, GOTEA analysis of the DkkMo versus no treatment groups highlighted several gene ontologies related to differentiation of T-cells, macrophages and erythrocytes (Fig6A). Co-expressed, functionally related gene modules and hub genes were also identified (FigS6) and plotted on volcano plots to visualize potential overlap between lists of DE genes (Fig6C\&D). As with the in vitro analyses, ALDH1A1 and Hsd17b4 were represented in both comparisons as hub and DE genes. Also of note were histone methyltransferases Setd7 and Nsd1, both implicated as mediators of oncogenesis ${ }^{34,35}$. IPA indicated that DkkMo treatment promoted upregulation of genes involved 
in necrosis and apoptosis in the case of both comparisons (Fig6E\&F). In the case of the no treatment versus DkkMo comparison, IPA also highlighted several gene groups associated with the death of blood cells, connective tissue cells and lymphocytes. Attrition of immune and stromal elements of the tumor was further supported by analysis using the Estimation of STromal and Immune cells in MAlignant Tumor tissues using Expression data (ESTIMATE) platform. When analyzed, DkkMo and to a greater extent, combination treated tumors, exhibited lower ESTIMATE values suggesting increased tumor purity. Collectively, the IPA and ESTIMATE data indicate that DkkMo may in part inhibit tumorigenesis by depleting the stroma as well as by modulating survival capacity.

\section{Discussion.}

The classical role of Dkk-1 is to target the LRP6/5 receptor to the Kremen2 receptor for internalization and destruction ${ }^{36}$, thereby inhibiting $\mathrm{cWnt}$ signaling by preventing the interaction of LRP5/6 and frizzled receptors. It is now widely accepted that cWnt signaling plays a major role in driving the differentiation of osteoprogenitors ${ }^{37}$, and as such, Dkk-1 is a potent osteoinhibitory factor. The first association between Dkk-1 and osteolytic malignant bone disease was demonstrated in $\mathrm{MM}^{12}$ then for juvenile $\mathrm{OS}{ }^{10}$ and skeletal metastases of breast, prostate and lung cancers ${ }^{13,14,38-40}$. Dkk-1 was subsequently shown to block the anabolic axis of bone turnover resulting in the premise that inhibition of Dkk-1 may slow the progression of MBD by inhibiting the development of osteolytic lesions. Initial strategies for the blockade of Dkk-1 began with antibodies, and these showed promise in MM models ${ }^{18}$ and in humans ${ }^{41}$, but administration of blocking antibodies must be given at relatively large doses given that systemic levels in humans can reach hundreds of ng.mL $\mathrm{m}^{-1}$ 10,12. To circumvent limitations of antibody therapy, this study employed a vivo morpholino (termed DkkMo) directed against human Dkk-1 
cDNA. Vivo morpholinos consist of an oligonucleotide mimetic attached to a dendrimeric moiety designed to facilitate cellular internalization. While originally utilized for gene knockdown in simple organisms, most notably zebrafish ${ }^{42}$, vivo morpholinos have been increasingly employed for gene knock-down in mammalian species such as rodents, dogs and even humans ${ }^{33}$. Indeed, an exon-skipping vivo morpholino was first successfully tested as a treatment for Duchenne Muscular Dystrophy (DMD) in $2009^{43}$ and shortly thereafter exon-skipping morpholinos Eteplirsen, Golodirsen, and Viltolarsen were approved for the treatment of DMD in the USA and Japan. DkkMo is a first generation vivo morpholino directed to the 5' coding region of human Dkk-1 [Fig1A]. When MOSJ-Dkk1 cells were exposed to DkkMo, human Dkk-1 secretion was dose-dependently inhibited [Fig1B] and the rate of accumulation of cells in culture was substantially reduced when compared to a control morpholino with scrambled sequence [Fig1F]. The reduction of cell expansion is probably attributed in part to enhanced susceptibility to nutritional stress resulting in cell death, an observation that is also made with the parental cell line MOS-J ${ }^{15}$. When confluent MOSJ-Dkk1 cells are treated with DkkMo, cell death readily occurs [Fig1D], phenocopying the parental and control cell lines, confirming that Dkk-1 is indeed required for enhanced survival. Resistance to stress is attributed to elevated expression of ALDH1A1 that occurs when Dkk-1 diverts Wnt signaling capacity from cWnt to a ncWnt pathway involving RhoA and JNK. Activated JNK triggers Jun to bind to the ALDH1A1 promoter, upregulating its expression. ALDH1A1 then acts to enhance resistance to nutritional and chemotherapeutic stressors in part by neutralization of reactive oxygen species ${ }^{15}$. The regulatory relationship between Dkk-1 and ALDH1A1 expression is demonstrated by the observation that when DkkMo is added to MOSJ-Dkk1 monolayers, ALDH1A1 transcription is significantly reduced [Fig2A]. The interrelated roles of ALDH1A1 and Dkk-1 in cell survival is 
further supported by HTS studies, where ALDH1A1 is identified as a DE and hub gene that is downregulated in the presence of DkkMo, coincident with the upregulation of several cell-death related processes. Unexpectedly, DkkMo also triggered cell death processes under mitotic as well as confluent conditions [Fig2B-G] and it is therefore reasonable to posit that DkkMo might have a detrimental effect on the survival of cells undergoing proliferation at the periphery of the tumor mass as well as the nutrient-deprived inner regions of a solid tumor.

From the in vitro HTS studies, two noteworthy hub and DE genes were identified with potential to contribute to tumor progression. Hsd17b4, encoding $17 \beta$-hydroxysteroid dehydrogenase/D-3-hydroxy acyl-coA dehydrogenase, was identified as a down-regulated hub gene under mitotic and confluent conditions when treated with DkkMo. Hsd17b4 is responsible for deactivation of estrogens and androgens as well as participating in peroxisomal betaoxidation of fatty acids and other complex substrates ${ }^{28}$. It is conceivable that it plays a role in oxidation of long chain or complex acids that result from the processing of reactive aldehydes by ALDH1A1. In confluent MOSJ-Dkk1 cells, DkkMo caused downregulation of hub transcript S1pr2, encoding sphingosine-1-phosphate receptor 2, regulator of osteoblast chemotaxis and inverse marker of osteoblast differentiation ${ }^{29}$.

Since DkkMo has the capacity to sensitize MOSJ-Dkk1 cells to stress, DkkMo may synergize with chemotherapeutics that induce oxidative damage in tumor cells. DRB is frequently employed as an adjuvant treatment for OS, and is known to cause oxidative damage to tissues and tumor cells through induction of ROS ${ }^{44}$. To examine whether expression of Dkk-1 mediates resistance to DRB, MOSJ-Dkk1 and MOSJ-pLenti, cells were exposed to DRB at a range of concentrations. While DRB had the capacity to blunt the expansion of both MOSJ lines, MOSJ-Dkk1 cells expanded more rapidly than MOSJ-pLenti cells at all doses [Fig3C]. 
Interestingly, the difference in $\mathrm{IC}_{50}$ values between MOSJ-Dkk1 cells and MOSJ-pLenti cells was greatest when the rates of proliferation were at their highest [Fig3B], suggesting that DRB is at its most toxic to cells undergoing proliferation and that Dkk-1 provides most protection at this time. Indeed, it is known that chromatin is susceptible to DRB-induced damage during mitosis ${ }^{44}$, Dkk-1 levels are elevated in cycling OS and mesenchymal cells ${ }^{45,46}$, and observations from this study indicates blockade of Dkk-1 during MOSJ-Dkk1 proliferation down-regulates processes related to cell accumulation [Fig2F]. When MOSJ-Dkk1 cells at various stages of culture were incubated with DRB, the expected degree of cell death was observed. In identical cultures with DkkMo added at $2.5 \mu \mathrm{M}$, additional cell death was observed in day 4 and 6 cultures where the rate of mitosis was at its highest [Fig3D]. Strikingly, when the dose of DkkMo was raised to 5 $\mu \mathrm{M}$, complete cell death was observed in all cultures in the presence of DRB. When $5 \mu \mathrm{M}$ DkkMo was added to cultures without DRB, the extent of cell loss was equivalent or greater than $\mathrm{DRB}$ alone at the $\mathrm{LD}_{50}$. The greatest reduction in cell number was observed in day 4 cultures, corresponding to the initiation of the proliferative phase, further supporting a role for Dkk-1 in protection of OS cells during proliferation. Given the key role of cWnt in the stimulation of proliferation in many stem cell systems, and in many cancers ${ }^{47}$, a pro-tumorigenic role for Dkk1 is surprising, but quite feasible given the multiple roles for $\mathrm{cWnt}$ in tissues and in organisms, the countless variants of Wnts and accessory molecules and the various non-canonical pathways. Indeed, there are several reported examples of Dkk-1 serving a pro-tumorgenic role through enhancement of growth and/or survival ${ }^{15,46,48,49}$, migratory ${ }^{50}$, and metastatic ${ }^{19,46}$ characteristics. Of particular note, Colla et al. ${ }^{51}$ demonstrated upregulation of Dkk-1 by human MM cells when challenged by ROS generated by chemotherapeutics thalidomide and lenalidomide, and while the study did not directly demonstrate upregulation of ALDH1A1 in this case, stimulation of JNK 
and Jun-C was observed. While Dkk-1 cannot be formally regarded as a proto-oncogene, the data herein collectively suggest that Dkk-1 certainly appears to serve as a survival factor for tumors during proliferative stress, especially when combined with chemotherapeutic challenge.

Orthotopically implanted MOSJ-Dkk1 cells rapidly generated osteolytic tumors in nude mice. When DRB was administered at a high dose, the rate of tumor expansion was not significantly affected, whereas DkkMo administered as a single agent significantly blunted tumor growth. Given that a robust degree of synergy was observed between DRB and DkkMo in in vitro experiments [Fig3D], it is surprising that the rate of tumor expansion was not substantially reduced as compared to single agents, but this was not the case. Indeed, even the formation of necrotic foci was not substantially enhanced by combination treatment, with significant foci observed in tumors from combination and DkkMo-treated groups [Fig4F]. One explanation for the lack of expected synergy is the possibility that DRB triggers expression of endogenous Dkk1 from MOSJ cells and this in turn competes with DkkMo, raising ALDH1A1 expression. While DRB did indeed upregulate endogenous Dkk-1 transcription by about 30\% [FigS1E], the secretion of murine Dkk-1 is negligible compared to the recombinant human form expressed by MOSJ-Dkk1 cells ${ }^{15}$, ALDH1A1 is not upregulated by DRB alone in MOSJ-Dkk1 cells [FigS1C-E], and the sequence of DkkMo is unlikely to cross react with murine Dkk-1 transcripts [Fig1A]. The in vitro assays employed, however, do not take into account potential sources of Dkk-1, ALDH1A1 or additional stress response agents derived from the tumor stroma. Dkk-1 is a potent inhibitor of osteogenic differentiation and key driver of osteolytic lesion formation in MBD ${ }^{12}$. Unlike parental MOSJ-cells, MOSJ-Dkk1 cells form highly osteolytic tumors in nude mice accompanied by significant bone deformation. Standard volumetric scans have the capacity to detect bone loss, but not necessarily deformations in bone 
topology that also contribute to the pathology of the disease. We therefore employed 3D reconstructions of $\mu \mathrm{CT}$ scans to perform quantitative topological comparisons between the contralateral and tumor-bearing tibiae [Fig4A]. These measurements confirmed that untreated MOSJ-Dkk1 tumors and those treated with DRB caused substantial deformation of adjacent bones which could be measured by quantifying deviations in the surface topology of the tumor laden tibia when compared to the unaffected contralateral side. When deviations were plotted, the data appeared as broad, asymmetrical distribution curves. When tumors were treated with DkkMo alone or in combination with DRB, the curves were narrower and symmetrical, with characteristics of a healthy topological profile [Fig5B]. When plots were analyzed statistically, DkkMo groups had significantly reduced bone deformation, but this was less evident for combination therapy suggesting that DRB may counteract, to a limited degree, the protective effects of DkkMo. This is possible given that DRB has a potent anti-mitotic effect and osteoblasts perform their reparative functions in part through localized bursts of proliferation ${ }^{52}$. Standard volumetric scans and mineral density measurements of tibiae and fibulae confirmed these findings. While immunological blockade of Dkk-1 has demonstrated bone rescue in experimental ${ }^{18}$ and clinical osteolytic MM ${ }^{41}$, this is the first time blockade of Dkk-1 has been shown to preserve topology as well as rescue bone loss. In this study, the small animal models employed precluded meaningful studies of gait and limb functionality, and as such, further studies of DkkMo in weight-bearing models are warranted.

Tumors were excised and subjected to HTS to gain mechanistic insights into the effect of DkkMo administration in the presence and absence of DRB. The dsRed label was utilized to exclude as much extraneous tissue as possible, but tumors harbored host tissue infiltrates such as immune cells, blood vessels and stroma. As expected, DkkMo upregulated cell death-related 
gene ontologies [Fig6A\&B], with ALDH1A1 and Hsd17b4 both identified as down-regulated DE transcripts and as hub genes [Fig6C\&D]. The consistent detection of ALDH1A1 and Hsd17b4 as down-regulated sequences with DkkMo treatment, and their potentially interrelated roles in the metabolism of complex organic substrates, suggests a novel role for Hsd17b4 as a mediator of drug resistance. Unexpectedly, there was significant representation from immune and hematopoietic gene ontology terms with DkkMo treatment, implying that Dkk-1 might also regulate immune processes within the MOSJ-Dkk1 tumor stroma. Indeed, Dkk-1 has been reported to stimulate infiltration of inflammatory macrophages and neutrophils and polarization of T-cells in acute lung injury ${ }^{53,54}$ and stimulate immunosuppressive myeloid suppressor cells in tumors so as to facilitate evasion from immune surveillance ${ }^{55}$. While the data herein do not exclusively support a predominantly inflammatory or immunoinhibitory role for Dkk-1, it is reasonable to predict that its inhibition is likely to contribute positively to outcome given the status of the current literature ${ }^{56}$. The IPA data highlighted upregulation of sequences associated with the death of immune, lymphatic, connective tissue upon treatment with DkkMo [Fig6E] suggesting that Dkk-1 blockade might be contributing to depletion of the tumor stroma. To explore this hypothesis more closely, the ESTIMATE platform was employed to assess tumor purity and it was apparent that the DkkMo and combination groups exhibited a greater level of predicted purity as compared to the no treatment and DRB groups [Fig6G]. It has recently been reported that the calculated purity of OS tumors, and thus a relatively low proportion of stroma, is inversely proportional to the potential for epithelial to mesenchymal transition (EMT), a process which is strongly related to disease progression and poorer prognosis ${ }^{57}$. Therefore, the data indicated that DkkMo may also act to reduce tumor progression through depletion of host stromal elements that contribute to EMT. 
Small interfering RNA has been employed to block Dkk-1 and exhibit neuroprotection in a model of intracerebral hemorrhage ${ }^{58}$, inhibit inflammation in a model of rheumatoid arthritis

${ }^{59}$, and in a model of hormone deficiency induced bone loss ${ }^{60}$, but at the time of publication, and to the best of our knowledge, this is the first demonstration of the use of a Dkk-1 targeting vivo morpholino to treat experimental MBD. Using a murine model of an aggressive Dkk-1 expressing osteolytic OS, we demonstrated that DkkMo had the capacity to reduce tumor progression through reduction of cell proliferation, perturbation of survival mechanisms, inhibition of bone destruction and preservation of topology, and potentially through depletion of the tumor stroma. Given that morpholinos have a robust half-life in vivo and are well tolerated, DkkMo represents a promising approach for the improvement of OS treatment in humans.

\section{Methods.}

Tissue Culture: Red fluorescent protein (RFP)-labeled MOSJ-Dkk1 and vector backbone control MOSJ-pLenti cells were generated as previously described ${ }^{15}$. MOSJ-Dkk1 cells are a murine osteochondral sarcoma cell line ${ }^{17}$ modified by the pLenti vector (Life Technologies, Carlsbad, CA) to constitutively express and secrete human Dkk-1 via the cytomegalovirus (CMV)

promoter. The control line, MOSJ-pLenti, harbors the vector backbone with no cDNA cassette. Both cell lines were cultured in medium containing alpha-minimal-essential-medium ( $\alpha \mathrm{MEM}$, Life Technologies) supplemented with $10 \%$ (v/v) fetal bovine serum (FBS, Atlanta Biologicals, Norcross, GA), $100 \mathrm{U} \cdot \mathrm{mL}^{-1}$ penicillin \& $100 \mu \mathrm{g} \cdot \mathrm{mL}^{-1}$ streptomycin (Life Technologies) and 2 mM L-glutamine (Life Technologies). For expansion, cells were seeded at 500 cells per $\mathrm{cm}^{2}$ with media changes every two days. Adherent cells were recovered using $0.25 \%(\mathrm{w} / \mathrm{v})$ 
trypsin/ethylene diamine tetra-acetic acid when a density of approximately $80-90 \%$ confluency was reached.

IC50 determination: To determine $\mathrm{DRB}_{\mathrm{IC}} \mathrm{I}_{0}$ for the MOSJ-Dkk1 and MOSJ-pLenti lines, cells were seeded at 1000 cells per well in 12-well plates. DRB was added 24 hours after seeding. Cells were exposed to 8 doses of varying DRB concentration in $\log _{10}$ increments. Cell number was determined using a hemocytometer and the Ecotox software package ${ }^{61}$ was used for $\mathrm{IC}_{50}$ calculations their comparisons.

Enzyme linked immunosorbent assay (ELISA): Two-day conditioned media were collected and stored at $-20 \circ \mathrm{C}$. For assays, media were thawed on ice, vortexed for $30 \mathrm{~s}$ then diluted $(1$ in $10-1$ in 100) with PBS $0.05 \%(\mathrm{v} / \mathrm{v})$ Tween 20 with $1 \%(\mathrm{v} / \mathrm{v})$ bovine serum albumin (BSA) then before performing ELISA. Human Dkk-1 ELISA was performed using a standard procedure recommended by the manufacturer (Duoset ELISA, R\&D Systems).

Quantitative RT-PCR (qRTPCR): The High Pure RNA isolation kit (Roche Diagnostics) was used for total RNA extraction from the cells. Copy DNA synthesis was performed using Superscript III kit (Life Technologies). TaqMan gene expression assays (Applied Biosystems) were used to carry out qRTPCR. APDH, MRPL19 and RPS18 were selected as reference genes 62-65. Reference genes were combined using geometric averaging as single internal control gene ${ }^{63}$. Fold changes were calculated using the $2^{-\Delta \Delta \mathrm{CT}}$ method ${ }^{66}$.

In vivo model of osteolytic OS: MOSJ-Dkk1 cells were employed in the OS xenograft model as described $^{15}$. Briefly, $1 \times 10^{6}$ MOSJ-Dkk1 cells were administered to two month-old immune compromised nude mice (Foxn1/Foxn1', Jackson laboratories) in $50 \mu \mathrm{L}$ of human plasma (Sigma, St. Louis, MO), with clotting activated facilitated by the addition of $50 \mu \mathrm{L}$ of 
thromboplastin A (Sigma). The cells were injected medially, into the interosseous space between the tibia and fibula. Tumor expansion was monitored three times a week in live animals $(\mathrm{n}=10$ per group) by detection of the RFP signal via live animal imaging using an IVIS Lumina III live animal imager (Perkin Elmer, Waltham, MA). For image analysis, photon flux from the tumors $\left(\mathrm{cm}^{-2} \cdot \mathrm{s}^{-1}\right)$ was calculated for each mouse by using a fixed size circular region of interest (ROI) encompassing the back limbs of the mouse in both supine and prone position. Treatments were initiated in animals harboring tumors with an approximate volume of $15 \mathrm{~mm}^{3}$ (approximately 3 mm diameter) as estimated by fluorescence imaging. DRB (5 mg.kg-1), DKKMo (12.5 mg. $\left.\mathrm{kg}^{-1}\right)$ or a combination of the two was administered by intraperitoneal injection every 2 days. The dose of DRB was empirically established to be the maximum sub-lethal dose, and the concentration of DkkMo was recommended from previous studies safely utilizing morpholinos in mice ${ }^{67,68}$. During the treatment period, photon flux values were scaled to a comparable background value, then normalized to the value obtained at the first administration of treatment, ensuring all mice had an arbitrary starting signal of 0 . Tumor growth rate was characterized by analyzing the delta photon flux over delta time. Slopes were compared using a mixed-model for repeated measures (MMRM) approach ${ }^{69,70}$.

Micro-CT $(\boldsymbol{\mu C T})$ scanning: Once euthanized, mice were fixed by trans-cardiac perfusion. All samples ( $\mathrm{n}=10$ each group) were scanned using a SkysScan1275 system with the filtered (1.5 mm aluminum) beam set to $40 \mathrm{kV}, 250 \mu \mathrm{A}$ and image capture set to $11 \mu \mathrm{m}$ resolution. Throughout the study, smoothing and beam hardening were fixed at 2\% (smoothing kernel gaussian) and 25\%, respectively. Ring artifact reduction and misalignment correction were adjusted manually to minimize scan artifacts. The dynamic range was set to between -1000 and 7519.5 HU for all reconstructions. Bone loss was calculated by comparison of volumes and bone 
mineral densities with the contralateral side. Bone mineral density (BMD) was measured using the attenuation coefficient method with calcium hydroxyapatite phantoms (Bruker) as calibrants. The mean of three $0.1 \mathrm{~mm}^{3}$ regions corresponding to $25 \%, 37 \%$ and $50 \%$ of the total length of the bones beginning from the proximal end was measured. Half of the specimens were also contrast stained with iodine/potassium iodide (IKI) for two weeks ${ }^{71}$, then rescanned under the same conditions to facilitate detection of soft tissues. These scans permitted accurate quantification of tumor volume.

Bone deformation indices: 3D Slicer software ${ }^{72}$ was used for bone volume and deformation measurements. Three dimensional renderings of the tumor-bearing and contralateral fibulae were constructed using the Otsu thresholding method (-1000 to $7519.5 \mathrm{HU})$, and the resultant bone model was then mirrored for registration. The mirrored model was computationally superimposed to contralateral bone model using a rigid algorithm to at least $5 \%$ accuracy. Models were further trimmed to the same anatomical landmarks. Model to model distances were calculated with the signed closest point approach. Bone deformation measurements were generated by quantifying the differences in coordinates between corresponding voxels in scans of the tumor-bearing versus contralateral images. The deformation analysis was done by quantile functional regression for regression analyses of distributions ${ }^{73}$. In short, quantlet, a union set of elements was selected from the sample distribution to represent the whole distribution by lasso regression and cross-validated concordance. The quantlet from each sample was then fit to a quantlet space model using a Bayesian modeling approach. Distribution plots were analyzed for differences between one another using the using the Quantile Function on Scalar Regression Analysis for Distributional Data method ${ }^{74}$ 
High throughput sequencing: For proliferative cultures, MOSJ-Dkk1 cells were seeded at 500 cells per $\mathrm{cm}^{2}$ in $175 \mathrm{~cm}^{2}$ flasks and allowed to enter the logarithmic stage of growth after 6 days of culture with changes of media every 2 days. Groups $(n=3)$ were no morpholino, $5 \mu \mathrm{M}$ DkkMo, $5 \mu \mathrm{M}$ scrambled morpholino control (scrMo) which was added $24 \mathrm{~h}$ after seeding. For the nutritional stress cohort, cells were seeded and cultured in the same manner, but exposed to $48 \mathrm{hr}$ of confluence without media change for 4 days. Cells were collected after one week of treatment. In all cases, cells were recovered by trypsinization, washed in PBS, and stored as flash frozen pellet $\left(1 \times 10^{6}\right.$ cells $)$ in liquid nitrogen until mRNA extraction.

For tumor sequencing, mice were initially fixed by trans-cardiac perfusion and stored in phosphate-buffered $3 \%$ formaldehyde with $10 \mathrm{mM} \mathrm{CaCl}_{2}$ at $4^{\circ} \mathrm{C}$. Four specimens from each group were randomly selected for RNA sequencing. Tumor/stroma was carefully dissected from bone and muscle tissue. Samples are sequenced by BGI Genomics (BGI Americas Corp. Cambridge, MA 02142, USA).

High throughput sequencing was performed on a NovaSeq 6000 system, recovering 150 million raw reads per sample in $150 \mathrm{bp}$ paired-end format. The reads were aligned to the GRCm38.98 reference genome using HISAT2 v 2.1.0 ${ }^{75}$. Gene counts were obtained by using SAMtools v.1.9 $9^{76}$ and featureCounts (Subread v.2.0.0) ${ }^{77,78}$. The downstream differential expression analysis was performed in R (Core Team, 2020) ${ }^{79}$ and with the DEseq2 package ${ }^{80}$. Weighted correlation network analysis was done with the WGCNA package ${ }^{81}$. Soft threshold was applied based on WGCNA manual suggestion. Hub genes were identified as top 10 correlated gene in gene significance cluster. Cytoscape ${ }^{82}$ was used for network visualization. The functional analyses were performed with the Ingenuity Pathway Analysis Platform (IPA, 
Qiagen, Redwood City, CA). FPKM was calculated using DEseq2 as part of the IPA input. Gene ontology analysis was performed on PANTHER ${ }^{83,84}$.

Histology: Specimens were stored in 3\% formaldehyde in PBS with $10 \mathrm{mM} \mathrm{CaCl}_{2}$ at $4^{\circ} \mathrm{C}$.

Decalcification was achieved with $1 \mathrm{M}$ dibasic EDTA, pH 8.0. The solution was changed every 2 days. The samples were then dehydrated through increasing gradients of alcohols, followed with Sub-X clearing agent (Surgipath Medical Industries, Richmond, IL) and embedded in paraffin (Richard-Allan Scientific, San Diego, CA). Paraffin-embedded samples were cut to $9 \mu \mathrm{m}$ sections. Sections were baked onto the slides at $60^{\circ} \mathrm{C}$ for $1 \mathrm{~h}$, followed by deparaffinized with Sub-X, and rehydration. For H\&E staining, sections were stained in hematoxylin solution Gill number 3 (Sigma) and counterstained with 1\% (w/v) eosin Y (Thermo Fisher) before clearing and dehydration. Masson's trichrome staining was achieved using a commercially available kit (American Master Tech, Lodi, CA) following the manufacturer's instructions.

Neural net programming and utilization: A U-net architecture based Convolutional Neural Network (CNN) was built using TensorFlow v.2.3.0 ${ }^{85,86}$. The model was built with the Adam optimizer algorithm. One sample was selected by systematic random sampling from each treatment group and segmented manually. Tumor and necrotic tissue were segmented separately. In total, 2176 images were used for training (70\% random assigned) and testing (19.5\% random assigned) to construct the CNN model. Model accuracy (FigS4) was obtained by applying the model to the validating (10.5\% random assigned) dataset. The validated CNN model then was used to segment the rest of the CT scans.

Statistics: GraphPad Prism version 8.00 for Mac was used to plot data and carry out statistical analysis. Single means were compared using t-tests while multiple tests of means were carried 
out using one-way analysis of variance (ANOVA) and either Dunnett's or Tukey's post-test where appropriate. Specific statistical parameters are given in figure legends. The tumor growth rate is characterized by analysis of the slope of tumor fluorescence over time between groups. Slope changes compare to No treatment group were analyzed using a mixed-model for repeated measures (MMRM) approach. Restricted Maximum Likelihood (ReML) estimation was used. The MMRM model included the treatment group, time point, and treatment group-by-time point interaction as fixed effects; and animals within treatment group as random effects allowing induvial difference for tumor growth rate. An unstructured covariance structure was used to model the within-animal error. Distribution plots were analyzed for differences between one another using the using the Quantile Function on Scalar Regression Analysis for Distributional Data method ${ }^{74}$

\section{Figure Legends.}

Figure 1: DkkMo blocks Dkk-1 expression by MOSJ-Dkk1 cells resulting in inhibition of expansion in vitro. Panel a: Target sequence of DkkMo, position on the Dkk-1 mRNA and comparison with the mouse orthologue. Panel b: DkkMo dose-dependently reduces secretion of Dkk-1 by MOSJ-Dkk1 cells ( $\mathrm{n}=4)$. Panel c: Expansion of MOSJ-Dkk1 cells compared to control MOSJ-pLenti cells that do not express Dkk-1 (n=3). Panel d: DkkMo inhibits resistance to nutritional stress caused by extended durations of confluent culture. Panel e: Dkk-1 secretion by MOSJ-Dkk1 cells in the presence of DkkMo or scrMo during expansion in culture $(n=5)$. Panel f: Cell expansion in the presence of DkkMo or scrMo over time in culture (n=5). Panel g: Dkk-1 secretion normalized to cell number (legend as Panels e and f). 
Figure 2: DkkMo blocks ALDH1A1 transcription, stress response and cell expansion pathways in MOSJ-Dkk1 cells in vitro. Panel a: Transcription of ALDH1A1 as measured by qRT-PCR by MOSJ-Dkk1 cells in the presence of DkkMo and scrMo $(n=3)$. Numbering refers to conditions where measurements differ with $\mathrm{p}<=0.05$. Panel b: GOTEA of RNA sequencing data with comparison between DkkMo and scrMo treatment of MOSJ-Dkk1 cells undergoing rapid proliferation $(n=3)$. Red font refers to gene ontologies that relate to cell death and survival, blue refers to osteogenic differentiation, green refers to immunoregulation and yellow refers to chemotaxis. Panel c: As panel b, but under extended durations of nutritional stress. Panel d: Volcano plot of DE genes after comparison of MOSJ-Dkk1 cells at log phase of growth in presence of DkkMo $(10 \mu \mathrm{M})$ or scrMo. Sequences that represent both DE and hub genes are labeled. Panel e: As panel d, but with MOSJ-Dkk1 cells subjected to extended confluence under nutritional stress. Panel f: Word cloud summarizing IPA results using DE gene lists from comparison of MOSJ-Dkk1 cells at log phase of growth in presence of DkkMo or scrMo. The words represent functional categories, the size of the font represents the size of the group, red and blue font represents upregulated and downregulated sequences respectively. Panel g: As panel f, but with MOSJ-Dkk1 cells subjected to extended confluence under nutritional stress.

Figure 3: Dkk-1 enhances resistance to challenge with DRB. Panel a: ID 50 calculations on MOSJ-Dkk1 and MOSJ-pLenti cells treated with various doses of DRB by time in culture. Panel b: Plot (bars) of the ratio of $\mathrm{IC}_{50}$ MOSJ-Dkk1 cells to MOSJ-pLenti cells when compared to the rate of expansion (lines). The greatest difference between the $\mathrm{IC}_{50}$ values occurs during the highest rate of cell division. Panel c: Growth curves of MOSK-Dkk1 cells and MOSJ-pLenti cells in the presence of various concentrations of DRB. Panel d: MOSJ-Dkk1 cell yields after 
treatment with DkkMo, scrMo, DRB or combinations. Numbering refers to conditions where measurements differ with $\mathrm{p}<=0.05$. P-values were calculated by the ratio-based test against a change in persistence method ${ }^{87}$.

Figure 4: DkkMo administration reduces the rate of expansion of MOSJ-Dkk1 tumors and causes generation of necrotic foci. Panel a: Fluorescent imaging of nude mice harboring orthotopic tumors of dsRed-labeled MOSJ-Dkk1 cells. Panel b: Plots of tumor expansion as a function of fold-change fluorescence intensity. Each line represents a single tumor $(\mathrm{n}=8-9)$. Pvalues calculated by mixed model regression and are compared to the no treatment condition. Panel c: Plots of animal weight throughout the the experiment $(n=8-9)$. Panel d: Axial images (above) of tumors at proximal/distal midpoint indicating larger numbers of radio-opaque foci in the DkkMo treated conditions (examples arrowed). Segmentation of the images (below) based on automated recognition by CNNA. Bone (white), soft tissue (green), tumor (blue) and foci (cyan) are indicated. Panel e: Volumetric measurements of radio-opaque foci using the CNNA $(n=5)$. Volumes per tumor (above) and the volume of necrotic foci normalized to tumor volume (below). The volumes per tumor (above) were analyzed by a generalized linear model account for tumor size. The volume of necrotic foci normalized to tumor volume (below) analyzed by beta regression $(*=\mathrm{p}<0.05)$ Panel f: $\mathrm{H}$ and $\mathrm{E}$ stained sections of tumors indicate that radiopaque structures are acellular foci of necrotic tissue (examples arrowed).

\section{Figure 5: DkkMo administration reduces the rate of bone destruction by MOSJ-Dkk1}

tumors. Panel a: Diagrammatic explanation of the principle of the topological analysis employed to measure bone remodeling. Panel b: Topological comparisons of tumor bearing 
tibiae with unaffected contralateral tibiae demonstrate DkkMo treatment preserves the original shape of the bone tissue. Rendered scans (above) indicate where surface of the tumor-laden tibia is inside (red) or outside (blue) the plane of the surface of the contralateral tibia. Distribution plots (below) with individual samples depicted in shades of brown, gray or green with the maximum width of the distribution depicted in pink. Healthy bones result in narrow distributions whereas damaged bones generate asymmetrical and broad distributions. P values represent statistical comparisons of plots versus no treatment. Panel c: Bone density for tumor laden tibiae and femora (leftmost two panels) plotted as deviation from the contralateral measurements. Bone absorption for tumor laden tibiae and femora (rightmost two panels) plotted as deviation from the contralateral measurements. P values calculated by ANOVA with Tukey multiple comparison test $(\mathrm{n}=8) . \mathrm{p}<0.05=*, \mathrm{p}<0.01=* *, \mathrm{p}<0.005=* * *, \mathrm{p}<0.001=* * * *$. Panel d: $\mathrm{H}$ and E stained sections of tumor bone interfaces indicate reduced bone erosion in DkkMo treated tumors. The bone $(b)$ and tumor $(t)$ are labeled in each case.

\section{Figure 6: DkkMo administration blocks stress response pathways in orthotopically} implanted MOSJ-Dkk1 tumors. Panel a: GOTEA of RNA sequencing data with comparison between DkkMo and no treatment (left). Panel b: As panel a, but with comparison of DRB and combination treatment (right) $(\mathrm{n}=4)$. Red font refers to groups related to cell death and survival and green font refers to immune-related genes. Panel c: Volcano plot of DE genes after comparison of the DkkMo with the no treatment group. Sequences that represent both DE and hub genes are labeled. Panel d: As panel C, but plot of DE genes after comparison of the DRB

group with the combination group. Panel e: Word cloud summarizing IPA results using DE gene lists from comparison of the DkkMo with the no treatment group. Color and font coding as in 
Figure 2. Panel f: As panel e, but with DE genes after comparison of the DRB group with the combination group. Panel g: Plot of ESTIMATE scores for each treatment group. Lower scores represent increased tumor purity and lower immune cell and stromal components.

Supplemental Figure 1: Panel a: Kaplan Meier survival plots for the calculation of the maximum tolerated DRB dose. Panel b: Plots of tumor expansion as a function of fold-change fluorescent intensity. Each line represents the mean fold-change fluorescence intensity. Individual points represent animals $(\mathrm{n}=8-9)$. Statistical analysis provided in Fig 4. Panel c: ALDH1A1 transcription measured by qRT-PCR calculated as $\Delta \mathrm{CT}$ in relation to aggregate measurements from a panel of standard reference genes (left) and as $\Delta \Delta \mathrm{CT}$ versus MOSJ-pLenti measurements at day 2 of culture. Panel d: ALDH1A1 transcription measured by qRT-PCR calculated as $\Delta \Delta \mathrm{CT}$ versus MOSJ-pLenti measurements at the appropriate day of culture. Panel e: Murine ALDH1A1, murine Dkk-1 and human Dkk-1 transcription in DRB treated and untreated MOSJ-Dkk1 cells at day 4 of culture measured by qRT-PCR calculated as $\Delta \mathrm{CT}$ versus pooled reference genes. For panel $\mathrm{c}$ and d, comparisons are versus MOSJ-Dkk1 group at each day in culture. P values calculated by ANOVA with Dunnett's post-test $(n=3) . p<0.05=$ *, $\mathrm{p}<0.01=* *, \mathrm{p}<0.005=* * *$.

Supplemental Figure 2: Diagrammatic summary of interrelationships between GOTEA and HUB analysis datasets. The orange panel indicates all shared DE genes, the blue panel indicates hub genes from log phase cultures and the pink panel indicates hub genes from cultures under nutritional stress. Black lines indicate functional interconnections between DE genes. 
Supplemental Figure 3: Panel a-c: H and E stained micrographs of untreated MOSJ-Dkk1 tumors $(10 \mathrm{x}$, bar $=200 \mu \mathrm{m}$, Panel a; 20x, bar $=100 \mu \mathrm{m}$ Panel b; and 40x, bar $=50 \mu \mathrm{m}$, Panel c) indicating homogeneous and highly proliferative spindle-shaped tumor cells with extensive bone involvement (Panel a). Panel d-f: $\mathrm{H}$ and E stained composite images of untreated (Panel d), DRB treated (Panel e), DkkMo treated (Panel f), or combination treated (Panel g) tumors. Asterisks indicate necrotic foci, $b a r=2 \mathrm{~mm}$.

Supplemental Figure 4: Panel a: Comparison of the automated segmentation of tumor and necrotic foci (predicted mask) with manual segmentation (true mask) and the input image. Panel b: Examples of automated segmentation for each of the treatment groups. Input image (left), segmented tumor (center) and combined image (right) are presented. In the rightmost image, bone (white), soft tissue (green), tumor (blue) and foci (cyan) are indicated. Panel c: Plot of accuracy as a function of epoch during the training of the neural net. At the conclusion of training, accuracy was in excess of $80 \%$.

Supplemental Figure 5: Panel a: H and E (above) or Masson's trichrome (below) stained composite micrographs of a combination treated tumor indicating collagenous infiltration of necrotic foci (blue stained areas with trichrome, asterisks. Bar $=2 \mathrm{~mm}$ ). Panel b: $\mathrm{H}$ and E (above) or Masson's trichrome (below) stained composite micrographs of necrotic lesions at higher power demonstrating that the collagen rich foci are essentially acellular (bar $=100 \mu \mathrm{m})$. Panel c: As Panel b, with indication of necrotic pyknosis (nuclear shrinkage) and nuclear fragmentation in cells adjacent to the foci. 
Supplemental Figure 6: Panel a: Summary of interrelationships between GOTEA and HUB analysis for in vivo HTS datasets. Categories in the red and green circles represent gene ontologies generated for the DkkMo versus NT and DRB versus combination comparisons respectively. The blue panel indicates hub genes generated form the DkkMo versus no treatment comparison, the pink panel indicates hub genes from the combination versus DRB comparison and the orange panel indicates shared DE genes. Black lines indicate functional interconnections between DE genes, hub genes and gene ontologies.

Acknowledgements: This work was funded by a High-Risk High Return and an Investigator Initiated Grant from the Cancer Prevention and Research Institute of Texas.

\section{Author contributions:}

SP, CAG: Conceptualized the research, performed the experiments, interpreted the data, wrote the manuscript.

SP, MC, CG, CC, CS, KB, HB, SW, JD: Performed experiments, performed data interpretation, wrote some sections of the manuscript.

RP: Performed pathology and interpretation.

AD, SP: Performed statistical analyses, designed and executed computational analyses.

CAG: Conceptualized research, awarded financial support to perform the work.

ALL AUTHORS: Proofed and approved manuscript.

Conflict of Interest: The authors declare no competing interests.

\section{Data availability statement:}

Until archive is completed, all raw data is available to reviewers upon request.

All raw data and associated code will be provided in a Raw Data File as part of the supplemental data packet and uploaded to FigShare.com [Accession: $x x x$ ].

Sequencing data is available via the Gene Expression Omnibus [Accession: $x x x$ ]. 


\section{$\underline{\text { References. }}$}

1 Ottaviani, G. \& Jaffe, N. The epidemiology of osteosarcoma. Cancer Treat Res 152, 313, doi:10.1007/978-1-4419-0284-9_1 (2009).

2 Misaghi, A., Goldin, A., Awad, M. \& Kulidjian, A. A. Osteosarcoma: a comprehensive review. SICOT J 4, 12, doi:10.1051/sicotj/2017028 (2018).

3 Isakoff, M. S., Bielack, S. S., Meltzer, P. \& Gorlick, R. Osteosarcoma: Current Treatment and a Collaborative Pathway to Success. J Clin Oncol 33, 3029-3035, doi:10.1200/JCO.2014.59.4895 (2015).

4 Partridge, A. H. et al. Adherence and persistence with oral adjuvant chemotherapy in older women with early-stage breast cancer in CALGB 49907: adherence companion study 60104. J Clin Oncol 28, 2418-2422, doi:10.1200/JCO.2009.26.4671 (2010).

5 Guth, U., Myrick, M. E., Schotzau, A., Kilic, N. \& Schmid, S. M. Drug switch because of treatment-related adverse side effects in endocrine adjuvant breast cancer therapy: how often and how often does it work? Breast Cancer Res Treat 129, 799-807, doi:10.1007/s10549-011-1668-y (2011).

6 Lash, T. L., Fox, M. P., Westrup, J. L., Fink, A. K. \& Silliman, R. A. Adherence to tamoxifen over the five-year course. Breast Cancer Res Treat 99, 215-220, doi:10.1007/s10549006-9193-0 (2006).

7 Subramanian, S. \& Viswanathan, V. K. in StatPearls (2020).

8 Jun, A. Y. et al. Tetrahydrofurofuran-type lignans inhibit breast cancer-mediated bone destruction by blocking the vicious cycle between cancer cells, osteoblasts and osteoclasts. Invest New Drugs 32, 1-13, doi:10.1007/s10637-013-9969-0 (2014).

9 Wei, R. et al. Analyzing the prognostic value of DKK1 expression in human cancers based on bioinformatics. Ann Transl Med 8, 552, doi:10.21037/atm-20-3263 (2020).

10 Lee, N. et al. A potential role for Dkk-1 in the pathogenesis of osteosarcoma predicts novel diagnostic and treatment strategies. Br J Cancer 97, 1552-1559, doi:6604069 [pii] 10.1038/sj.bjc.6604069 (2007).

11 Wirths, O. et al. Overexpression of human Dickkopf-1, an antagonist of wingless/WNT signaling, in human hepatoblastomas and Wilms' tumors. Lab Invest 83, 429-434, doi:10.1097/01.lab.0000059926.66359.bd (2003).

12 Tian, E. et al. The role of the Wnt-signaling antagonist DKK1 in the development of osteolytic lesions in multiple myeloma. N Engl J Med 349, 2483-2494, doi:10.1056/NEJMoa030847349/26/2483 [pii] (2003).

13 Rachner, T. D. et al. High serum levels of Dickkopf-1 are associated with a poor prognosis in prostate cancer patients. BMC Cancer 14, 649, doi:10.1186/1471-2407-14-649 (2014).

14 Zhou, S. J., Zhuo, S. R., Yang, X. Q., Qin, C. X. \& Wang, Z. L. Serum Dickkopf-1 expression level positively correlates with a poor prognosis in breast cancer. Diagn Pathol 9, 161, doi:10.1186/s13000-014-0161-4 (2014).

15 Krause, U., Ryan, D. M., Clough, B. H. \& Gregory, C. A. An unexpected role for a Wntinhibitor: Dickkopf-1 triggers a novel cancer survival mechanism through modulation of aldehyde-dehydrogenase-1 activity. Cell Death Dis 5, e1093, doi:10.1038/cddis.2014.67 (2014). 16 Clark, D. W. \& Palle, K. Aldehyde dehydrogenases in cancer stem cells: potential as therapeutic targets. Ann Transl Med 4, 518, doi:10.21037/atm.2016.11.82 (2016). 
17 Joliat, M. J., Umeda, S., Lyons, B. L., Lynes, M. A. \& Shultz, L. D. Establishment and characterization of a new osteogenic cell line (MOS-J) from a spontaneous C57BL/6J mouse osteosarcoma. In Vivo 16, 223-228 (2002).

18 Yaccoby, S. et al. Antibody-based inhibition of DKK1 suppresses tumor-induced bone resorption and multiple myeloma growth in vivo. Blood 109, 2106-2111, doi:blood-2006-09047712 [pii] 10.1182/blood-2006-09-047712 (2007).

19 Goldstein, S. D., Trucco, M., Guzman, W. B., Hayashi, M. \& Loeb, D. M. A monoclonal antibody against the Wnt signaling inhibitor dickkopf-1 inhibits osteosarcoma metastasis in a preclinical model. Oncotarget, doi:10.18632/oncotarget.8522 (2016).

20 Awad, O. et al. High ALDH activity identifies chemotherapy-resistant Ewing's sarcoma stem cells that retain sensitivity to EWS-FLI1 inhibition. PLoS One 5, e13943, doi:10.1371/journal.pone.0013943 (2010).

21 Marchitti, S. A., Brocker, C., Stagos, D. \& Vasiliou, V. Non-P450 aldehyde oxidizing enzymes: the aldehyde dehydrogenase superfamily. Expert Opin Drug Metab Toxicol 4, 697 720, doi:10.1517/17425255.4.6.697 (2008).

22 Vasiliou, V. \& Nebert, D. W. Analysis and update of the human aldehyde dehydrogenase (ALDH) gene family. Hum Genomics 2, 138-143 (2005).

23 Makia, N. L., Bojang, P., Falkner, K. C., Conklin, D. J. \& Prough, R. A. Murine hepatic aldehyde dehydrogenase 1a1 is a major contributor to oxidation of aldehydes formed by lipid peroxidation. Chem Biol Interact 191, 278-287, doi:S0009-2797(11)00018-4 [pii] 10.1016/j.cbi.2011.01.013 (2011).

24 Rodriguez-Torres, M. \& Allan, A. L. Aldehyde dehydrogenase as a marker and functional mediator of metastasis in solid tumors. Clin Exp Metastasis 33, 97-113, doi:10.1007/s10585-015-9755-9 (2016).

25 Wang, L., Park, P., Zhang, H., La Marca, F. \& Lin, C. Y. Prospective identification of tumorigenic osteosarcoma cancer stem cells in OS99-1 cells based on high aldehyde dehydrogenase activity. Int J Cancer 128, 294-303, doi:10.1002/ijc.25331 (2011).

26 Greco, N. et al. ALDH Activity Correlates with Metastatic Potential in Primary Sarcomas of Bone. J Cancer Ther 5, 331-338, doi:10.4236/jct.2014.54040 (2014).

27 Douville, J., Beaulieu, R. \& Balicki, D. ALDH1 as a functional marker of cancer stem and progenitor cells. Stem Cells Dev 18, 17-25, doi:10.1089/scd.2008.0055 (2009).

28 de Launoit, Y. \& Adamski, J. Unique multifunctional HSD17B4 gene product: 17betahydroxysteroid dehydrogenase 4 and D-3-hydroxyacyl-coenzyme A dehydrogenase/hydratase involved in Zellweger syndrome. J Mol Endocrinol 22, 227-240, doi:10.1677/jme.0.0220227 (1999).

29 Meshcheryakova, A., Mechtcheriakova, D. \& Pietschmann, P. Sphingosine 1-phosphate signaling in bone remodeling: multifaceted roles and therapeutic potential. Expert Opin Ther Targets 21, 725-737, doi:10.1080/14728222.2017.1332180 (2017).

30 Honoki, K. et al. Possible involvement of stem-like populations with elevated ALDH1 in sarcomas for chemotherapeutic drug resistance. Oncol Rep 24, 501-505 (2010).

31 Carvalho, C. et al. Doxorubicin: the good, the bad and the ugly effect. Curr Med Chem 16, 3267-3285, doi:10.2174/092986709788803312 (2009).

32 Moulton, J. D. Guide for Morpholino Users: Toward Therapeutics. Journal of Drug Discovery, Development and Delivery 3, 1023 (2016). 
33 Moulton, J. D. \& Jiang, S. Gene knockdowns in adult animals: PPMOs and vivomorpholinos. Molecules 14, 1304-1323, doi:10.3390/molecules14031304 (2009).

34 Bennett, R. L., Swaroop, A., Troche, C. \& Licht, J. D. The Role of Nuclear ReceptorBinding SET Domain Family Histone Lysine Methyltransferases in Cancer. Cold Spring Harb Perspect Med 7, doi:10.1101/cshperspect.a026708 (2017).

35 Keating, S. T. \& El-Osta, A. Transcriptional regulation by the Set7 lysine methyltransferase. Epigenetics 8, 361-372, doi:10.4161/epi.24234 (2013).

36 Bianco, P. et al. The meaning, the sense and the significance: translating the science of mesenchymal stem cells into medicine. Nat Med 19, 35-42, doi:10.1038/nm.3028 (2013).

37 Teufel, S. \& Hartmann, C. Wnt-signaling in skeletal development. Curr Top Dev Biol 133, 235-279, doi:10.1016/bs.ctdb.2018.11.010 (2019).

38 Hall, C. L., Daignault, S. D., Shah, R. B., Pienta, K. J. \& Keller, E. T. Dickkopf-1 expression increases early in prostate cancer development and decreases during progression from primary tumor to metastasis. Prostate 68, 1396-1404, doi:10.1002/pros.20805 (2008).

39 Gregory, L. S., Choi, W., Burke, L. \& Clements, J. A. Breast cancer cells induce osteolytic bone lesions in vivo through a reduction in osteoblast activity in mice. PLoS One 8, e68103, doi:10.1371/journal.pone.0068103 (2013).

40 Chu, T., Teng, J., Jiang, L., Zhong, H. \& Han, B. Lung cancer-derived Dickkopf1 is associated with bone metastasis and the mechanism involves the inhibition of osteoblast differentiation. Biochem Biophys Res Commun 443, 962-968, doi:10.1016/j.bbrc.2013.12.076 (2014).

41 Iyer, S. P. et al. A Phase IB multicentre dose-determination study of BHQ880 in combination with anti-myeloma therapy and zoledronic acid in patients with relapsed or refractory multiple myeloma and prior skeletal-related events. Br J Haematol 167, 366-375, doi:10.1111/bjh.13056 (2014).

42 Corey, D. R. \& Abrams, J. M. Morpholino antisense oligonucleotides: tools for investigating vertebrate development. Genome Biol 2, REVIEWS1015, doi:10.1186/gb-2001-25-reviews1015 (2001).

43 Kinali, M. et al. Local restoration of dystrophin expression with the morpholino oligomer AVI-4658 in Duchenne muscular dystrophy: a single-blind, placebo-controlled, dose-escalation, proof-of-concept study. The Lancet. Neurology 8, 918-928, doi:10.1016/S1474-4422(09)70211X (2009).

44 Baxter-Holland, M. \& Dass, C. R. Doxorubicin, mesenchymal stem cell toxicity and antitumour activity: implications for clinical use. J Pharm Pharmacol 70, 320-327, doi:10.1111/jphp.12869 (2018).

45 Gregory, C. A., Singh, H., Perry, A. S. \& Prockop, D. J. The Wnt signaling inhibitor dickkopf-1 is required for reentry into the cell cycle of human adult stem cells from bone marrow. J Biol Chem 278, 28067-28078, doi:10.1074/jbc.M300373200 M300373200 [pii] (2003).

46 Thudi, N. K. et al. Dickkopf-1 (DKK-1) stimulated prostate cancer growth and metastasis and inhibited bone formation in osteoblastic bone metastases. Prostate 71, 615-625, doi:10.1002/pros.21277 (2011).

47 Clevers, H. \& Nusse, R. Wnt/beta-catenin signaling and disease. Cell 149, 1192-1205, doi:S0092-8674(12)00586-7 [pii]10.1016/j.cell.2012.05.012 (2012).

48 Matushansky, I. et al. Derivation of sarcomas from mesenchymal stem cells via inactivation of the Wnt pathway. J Clin Invest 117, 3248-3257, doi:10.1172/JCI31377 (2007). 
49 Hall, C. L. et al. p21CIP-1/WAF-1 induction is required to inhibit prostate cancer growth elicited by deficient expression of the Wnt inhibitor Dickkopf-1. Cancer Res 70, 9916-9926, doi:10.1158/0008-5472.CAN-10-0440 (2010).

50 Takahashi, N. et al. Dickkopf-1 is overexpressed in human pancreatic ductal adenocarcinoma cells and is involved in invasive growth. Int J Cancer 126, 1611-1620, doi:10.1002/ijc.24865 (2010).

51 Colla, S. et al. The oxidative stress response regulates DKK1 expression through the JNK signaling cascade in multiple myeloma plasma cells. Blood 109, 4470-4477, doi:blood-2006-11056747 [pii]10.1182/blood-2006-11-056747 (2007).

52 Einhorn, T. A. The cell and molecular biology of fracture healing. Clin Orthop Relat Res, S7-21 (1998).

53 Guo, Y. et al. Platelet-derived Wnt antagonist Dickkopf-1 is implicated in ICAM-

1/VCAM-1-mediated neutrophilic acute lung inflammation. Blood 126, 2220-2229, doi:10.1182/blood-2015-02-622233 (2015).

54 Chae, W. J. et al. The Wnt Antagonist Dickkopf-1 Promotes Pathological Type 2 CellMediated Inflammation. Immunity 44, 246-258, doi:10.1016/j.immuni.2016.01.008 (2016).

55 D'Amico, L. et al. Dickkopf-related protein 1 (Dkk1) regulates the accumulation and function of myeloid derived suppressor cells in cancer. J Exp Med 213, 827-840, doi:10.1084/jem.20150950 (2016).

56 Jaschke, N., Hofbauer, L. C., Gobel, A. \& Rachner, T. D. Evolving functions of Dickkopf-1 in cancer and immunity. Cancer letters 482, 1-7, doi:10.1016/j.canlet.2020.03.031 (2020).

57 Peng, Y. X. et al. EMT-related gene expression is positively correlated with immunity and may be derived from stromal cells in osteosarcoma. PeerJ 8, e8489, doi:10.7717/peerj.8489 (2020).

58 Li, Z. et al. Small Interfering RNA Targeting Dickkopf-1 Contributes to Neuroprotection After Intracerebral Hemorrhage in Rats. J Mol Neurosci 61, 279-288, doi:10.1007/s12031-0170883-3 (2017).

59 Liu, Y. Y. et al. Activity of fibroblast-like synoviocytes in rheumatoid arthritis was impaired by dickkopf-1 targeting siRNA. Chinese medical journal, 679-686, doi:10.1097/CM9.0000000000000697 (2020).

60 Wang, F. S. et al. Knocking down dickkopf-1 alleviates estrogen deficiency induction of bone loss. A histomorphological study in ovariectomized rats. Bone 40, 485-492, doi:10.1016/j.bone.2006.09.004 (2007).

61 Hlina BL, B. O., Robinson CS, Dhiyebi H, Wilkie MP. Changes in the sensitivity of piscicide in an invasive species. North American Journal of Fisheries Management (2019). 62 Abuna, R. P. F. et al. Selection of reference genes for quantitative real-time polymerase chain reaction studies in rat osteoblasts. J Cell Physiol 234, 749-756, doi:10.1002/jcp.26886 (2018).

63 Vandesompele, J. et al. Accurate normalization of real-time quantitative RT-PCR data by geometric averaging of multiple internal control genes. Genome Biol 3, RESEARCH0034, doi:10.1186/gb-2002-3-7-research0034 (2002).

64 Mandell, J. B. et al. Combination Therapy with Disulfiram, Copper, and Doxorubicin for Osteosarcoma: In Vitro Support for a Novel Drug Repurposing Strategy. Sarcoma 2019, 1320201, doi:10.1155/2019/1320201 (2019). 
65 Rienzo, M. et al. Identification of valid reference housekeeping genes for gene expression analysis in tumor neovascularization studies. Clin Transl Oncol 15, 211-218, doi:10.1007/s12094-012-0904-1 (2013).

66 Livak, K. J. \& Schmittgen, T. D. Analysis of relative gene expression data using realtime quantitative PCR and the 2(-Delta Delta C(T)) Method. Methods 25, 402-408, doi:10.1006/meth.2001.1262S1046-2023(01)91262-9 [pii] (2001).

67 Ferguson, D. P., Dangott, L. J. \& Lightfoot, J. T. Lessons learned from vivomorpholinos: How to avoid vivo-morpholino toxicity. Biotechniques 56, 251-256, doi:10.2144/000114167 (2014).

68 Morcos, P. A., Li, Y. \& Jiang, S. Vivo-Morpholinos: a non-peptide transporter delivers Morpholinos into a wide array of mouse tissues. BioTechniques 45, 613-614, 616, 618 passim (2008).

69 Detry, M. A. \& Ma, Y. Analyzing Repeated Measurements Using Mixed Models. JAMA 315, 407-408, doi:10.1001/jama.2015.19394 (2016).

70 Administration, F. a. D. Statistical Approaches to Establishing Bioequivalence. Guidance for Industry (2001).

71 Metscher, B. D. MicroCT for comparative morphology: simple staining methods allow high-contrast 3D imaging of diverse non-mineralized animal tissues. BMC Physiol 9, 11, doi:1472-6793-9-11 [pii]10.1186/1472-6793-9-11 (2009).

72 Fedorov, A. et al. 3D Slicer as an image computing platform for the Quantitative Imaging Network. Magn Reson Imaging 30, 1323-1341, doi:10.1016/j.mri.2012.05.001 (2012).

73 Yang, H., Baladandayuthapani, V., Rao, A. U. \& Morris, J. S. Regression Analyses of Distributions using Quantile Functional Regression. arXiv preprint arXiv:1810.03496 (2018).

74 Yang, H., Baladandayuthapani, V., Rao, A. U. K. \& Morris, J. S. Quantile Function on Scalar Regression Analysis for Distributional Data. J Am Stat Assoc 115, 90-106, doi:10.1080/01621459.2019.1609969 (2020).

75 Kim, D., Langmead, B. \& Salzberg, S. L. HISAT: a fast spliced aligner with low memory requirements. Nat Methods 12, 357-360, doi:10.1038/nmeth.3317 (2015).

76 Li, H. et al. The Sequence Alignment/Map format and SAMtools. Bioinformatics 25, 2078-2079, doi:10.1093/bioinformatics/btp352 (2009).

77 Liao, Y., Smyth, G. K. \& Shi, W. featureCounts: an efficient general purpose program for assigning sequence reads to genomic features. Bioinformatics 30, 923-930, doi:10.1093/bioinformatics/btt656 (2014).

78 Anders, S., Pyl, P. T. \& Huber, W. HTSeq--a Python framework to work with highthroughput sequencing data. Bioinformatics 31, 166-169, doi:10.1093/bioinformatics/btu638 (2015).

79 Team, R. C. R: A Language and Environment for Statistical Computin. (2020).

80 Love, M. I., Huber, W. \& Anders, S. Moderated estimation of fold change and dispersion for RNA-seq data with DESeq2. Genome Biol 15, 550, doi:10.1186/s13059-014-0550-8 (2014).

81 Langfelder, P. \& Horvath, S. WGCNA: an R package for weighted correlation network analysis. BMC Bioinformatics 9, 559, doi:10.1186/1471-2105-9-559 (2008).

82 Shannon, P. et al. Cytoscape: a software environment for integrated models of biomolecular interaction networks. Genome Res 13, 2498-2504, doi:10.1101/gr.1239303 (2003). 83 Thomas, P. D. et al. PANTHER: a library of protein families and subfamilies indexed by function. Genome Res 13, 2129-2141, doi:10.1101/gr.772403 (2003). 
84 Thomas, P. D. et al. Applications for protein sequence-function evolution data: mRNA/protein expression analysis and coding SNP scoring tools. Nucleic Acids Res 34, W645650, doi:10.1093/nar/gk1229 (2006).

85 Ronneberger, O., Fischer, P. \& Brox, T. 234-241 (Springer International Publishing).

86 Abadi, M. et al. Tensorflow: Large-scale machine learning on heterogeneous distributed systems. arXiv preprint arXiv:1603.04467 (2016).

87 Hlina, B., Birceanu, O., Robinson, C., Dhiyebi, H. \& Wilkie, M. Seasonal variation in the sensitivity of invasive sea lampreys to the lampricide TFM: Importance of energy reserves and temperature. N. Am. J. Fish. Manag 28, 1609-1617 (2008). 


\section{Figure 1.}

a.

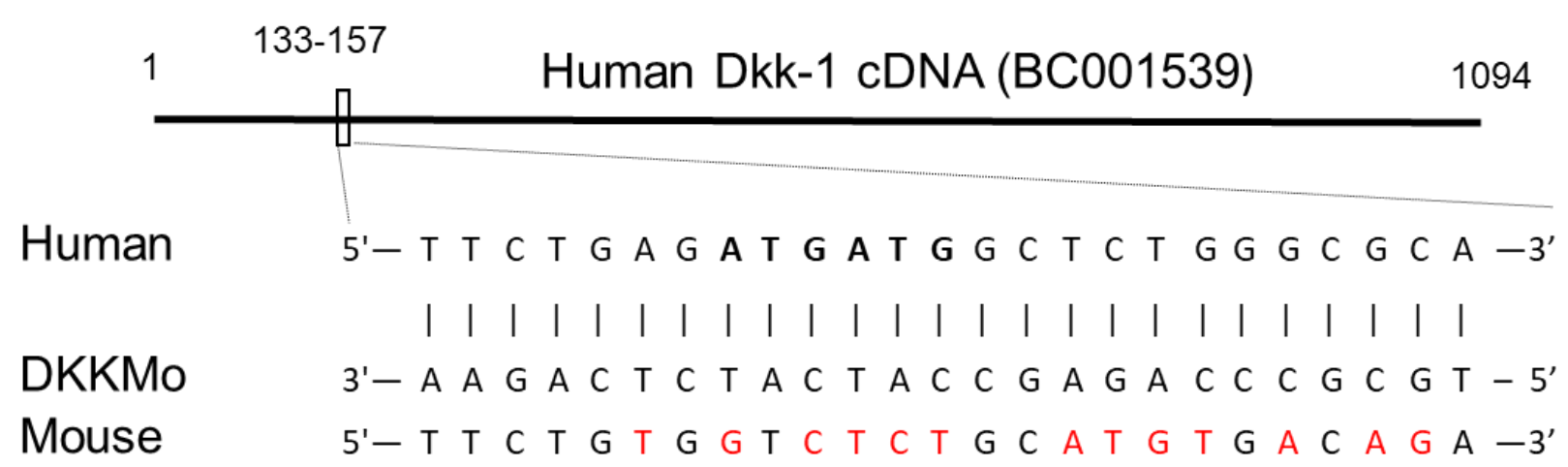

b.

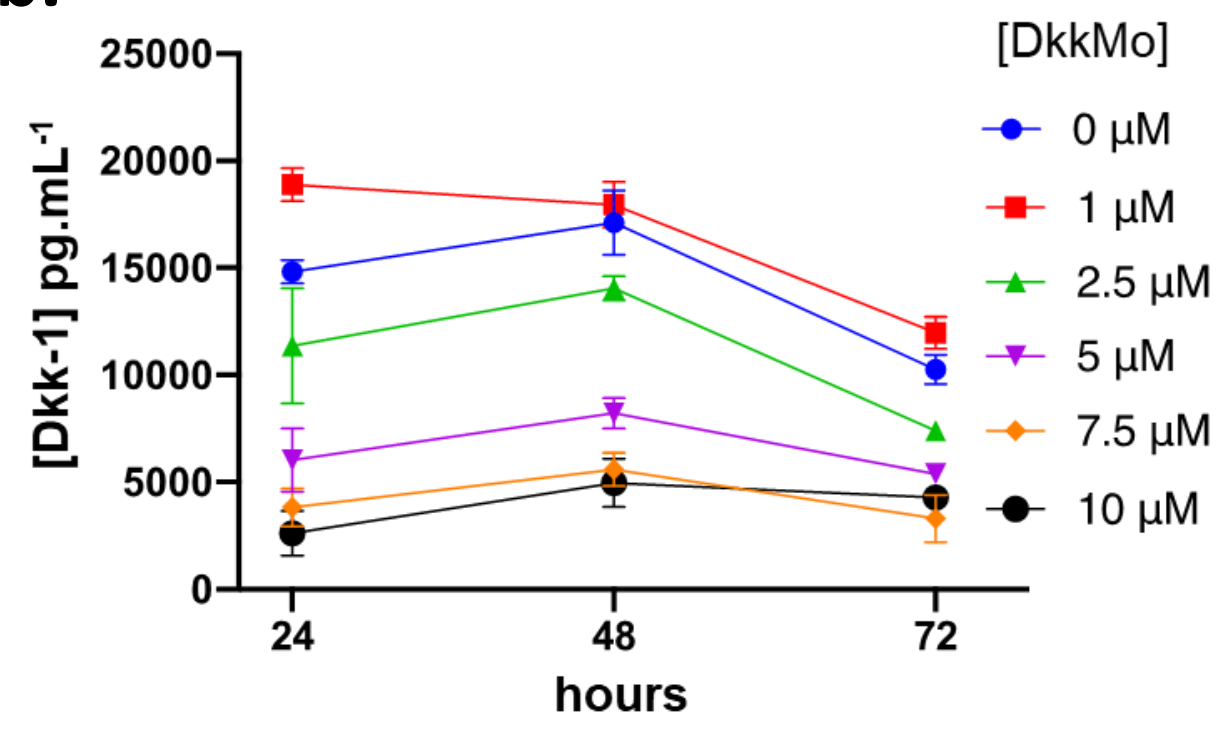

c.

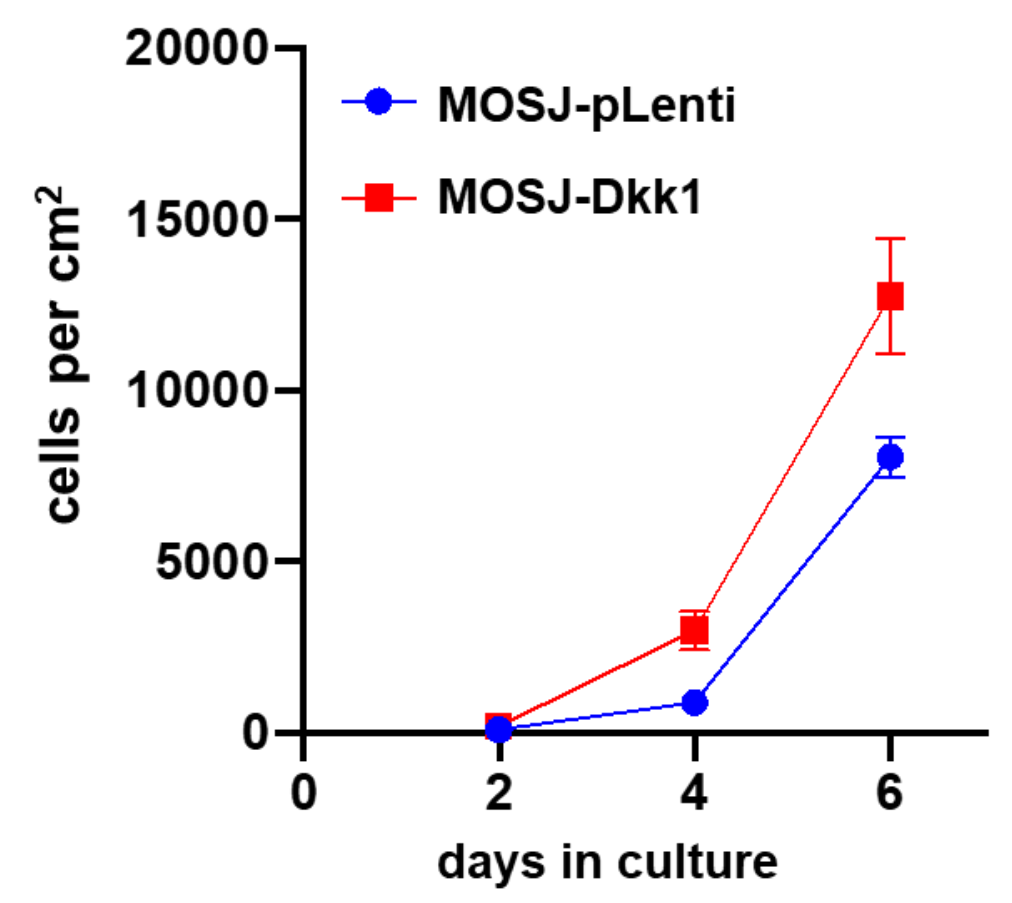

d.

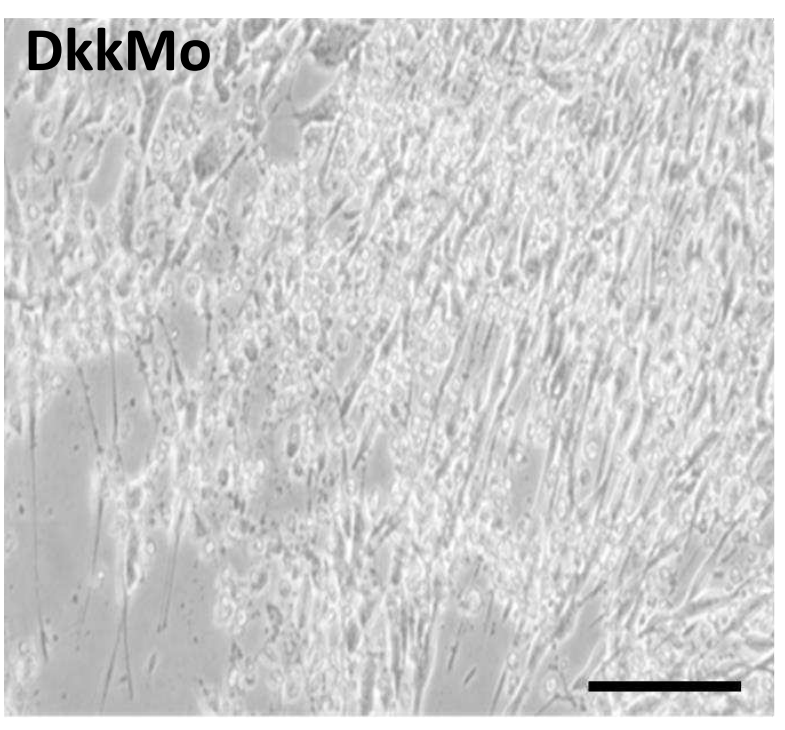

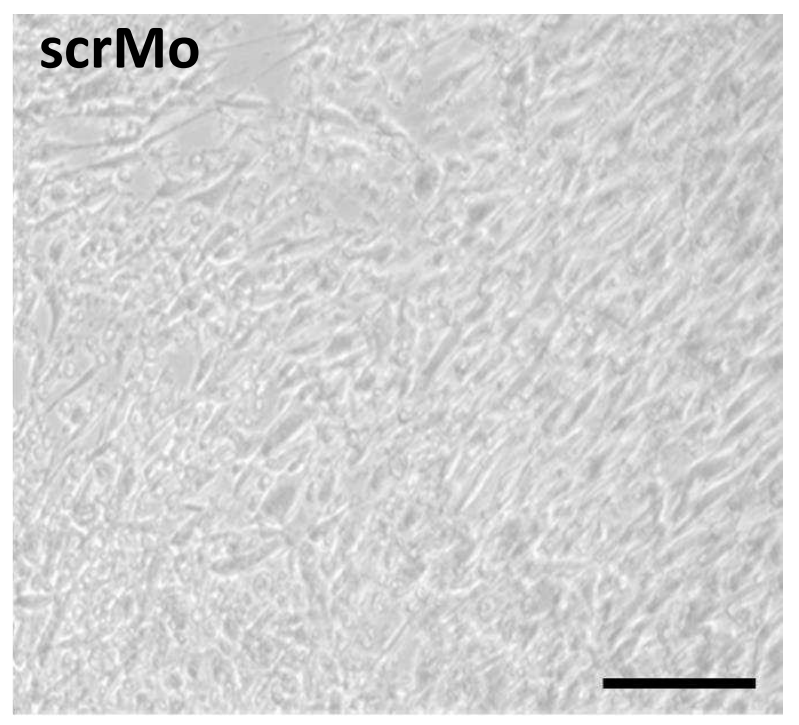

e.

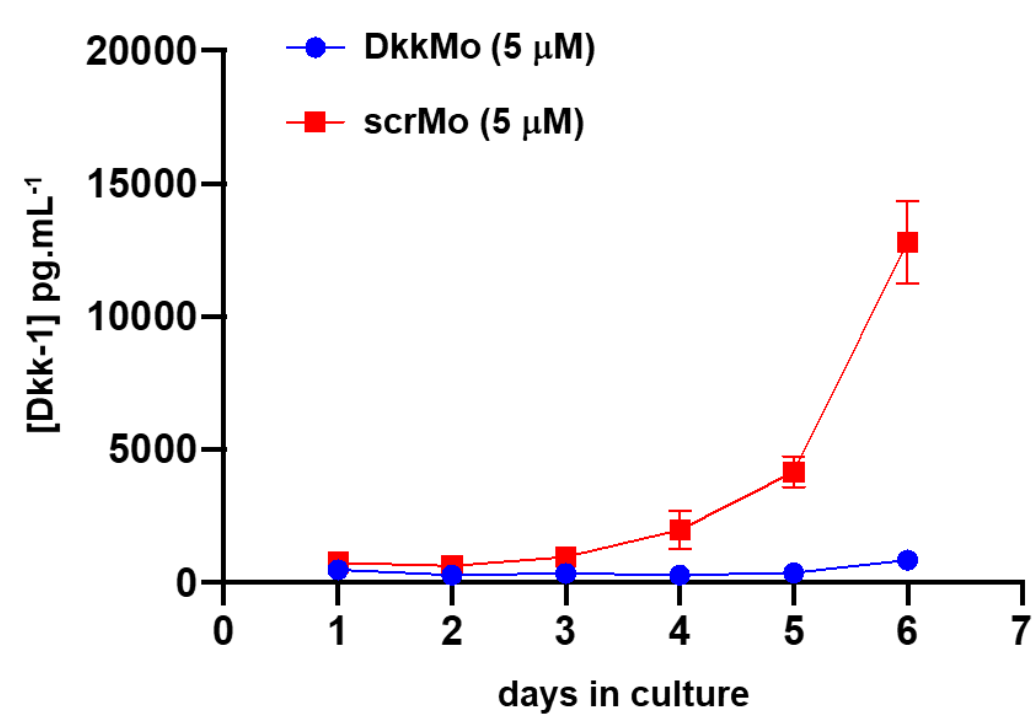

f.

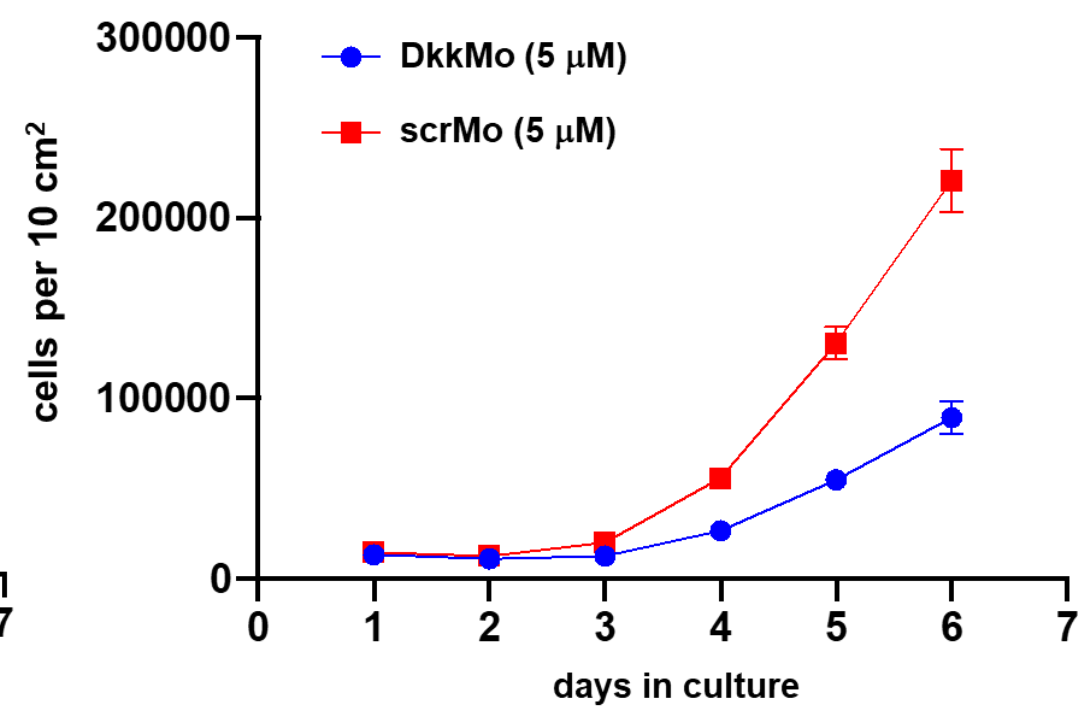

g.

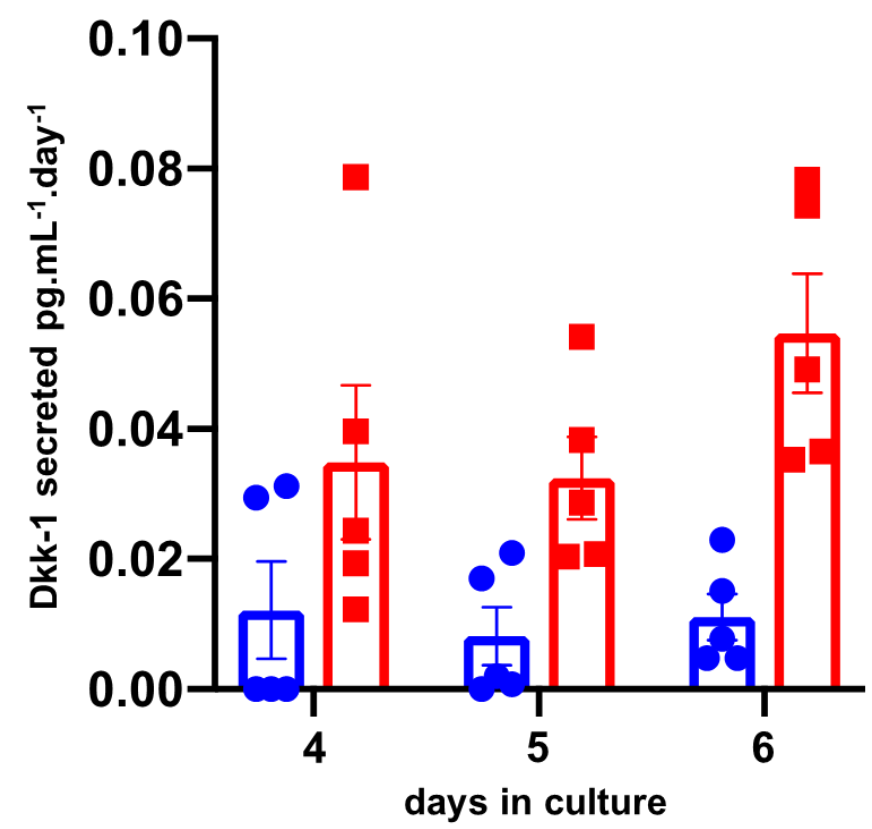


Figure 2.

a.
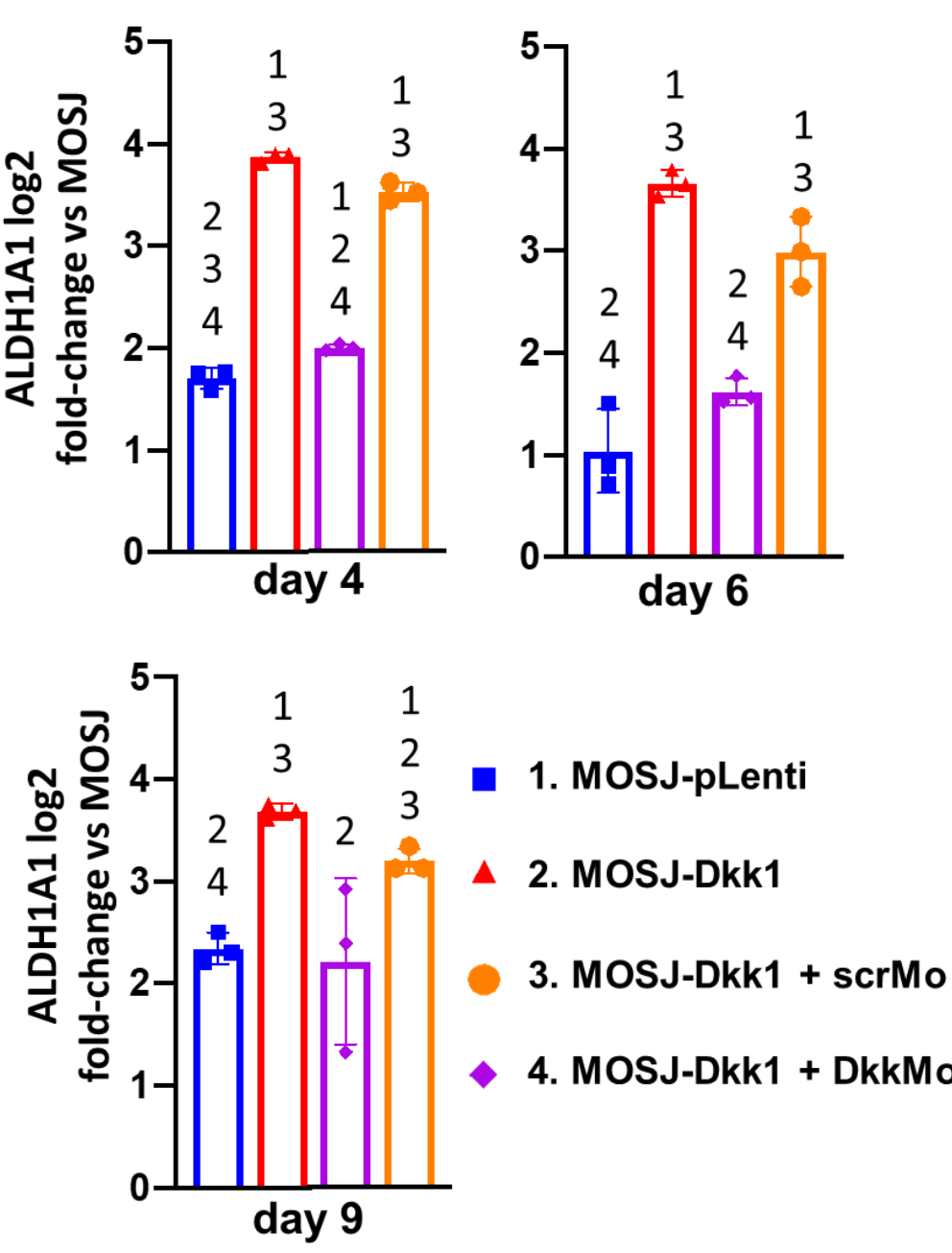

b.

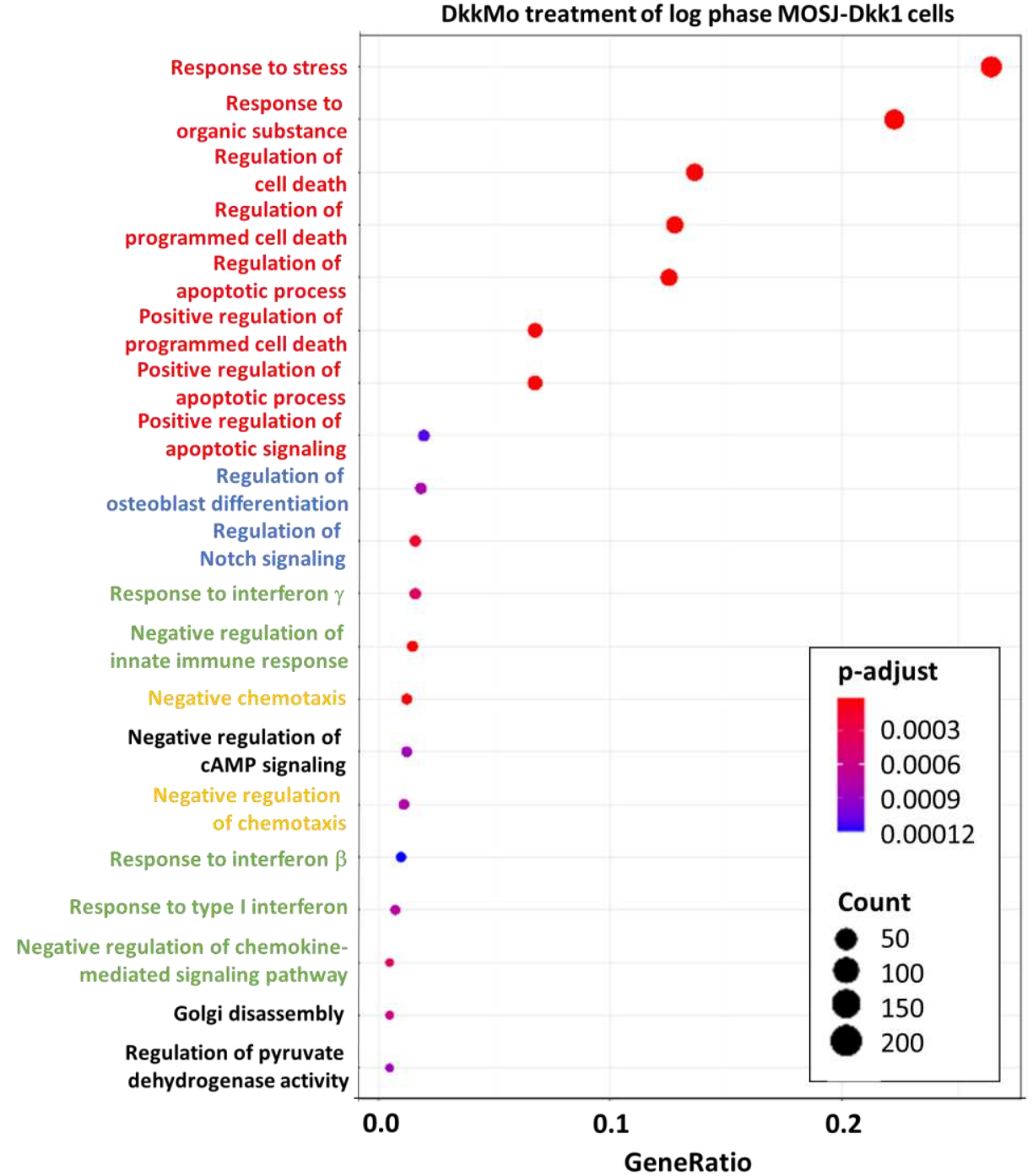

C.

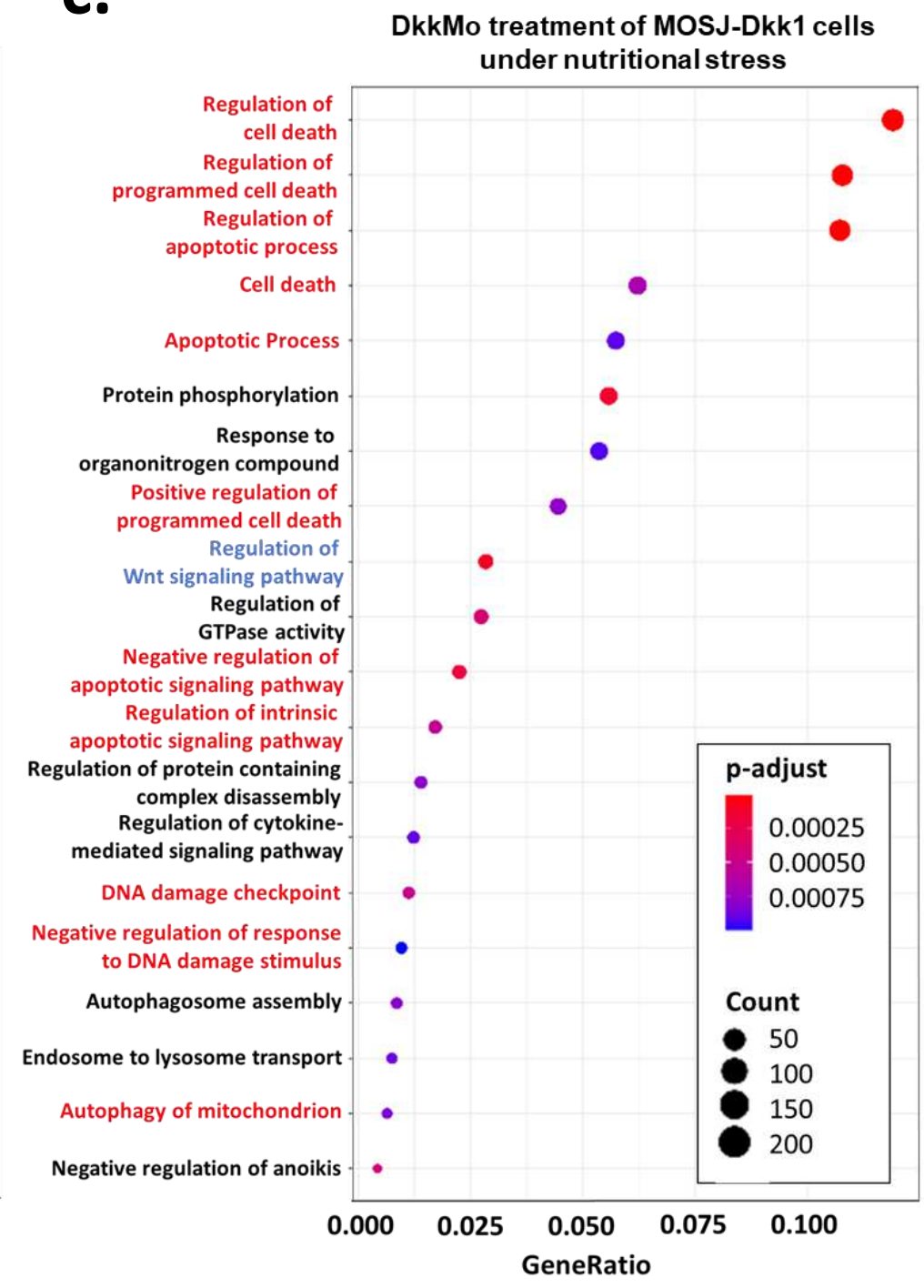

d.

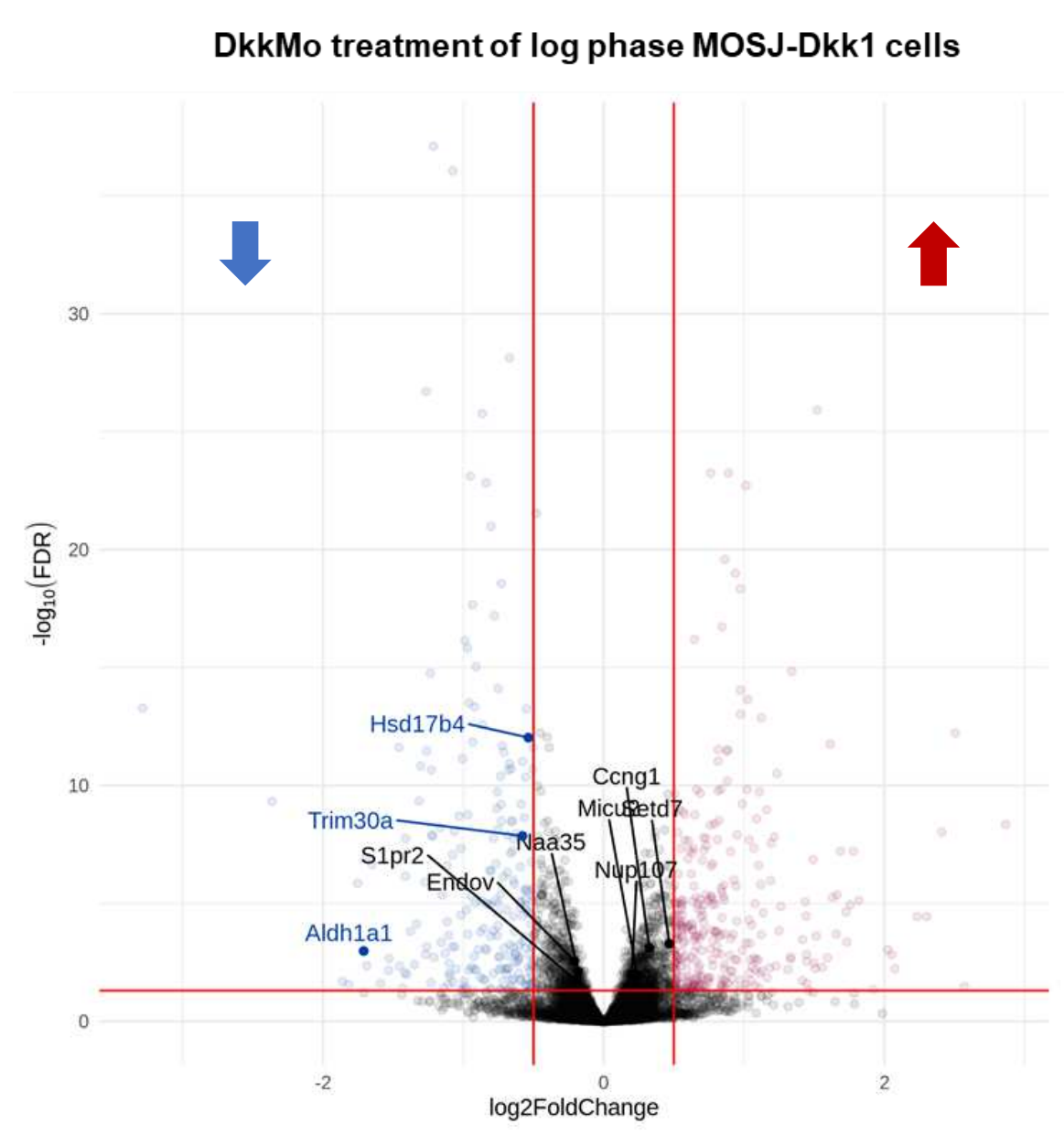

e.

DkkMo treatment of MOSJ-Dkk1 cells under nutritional stress

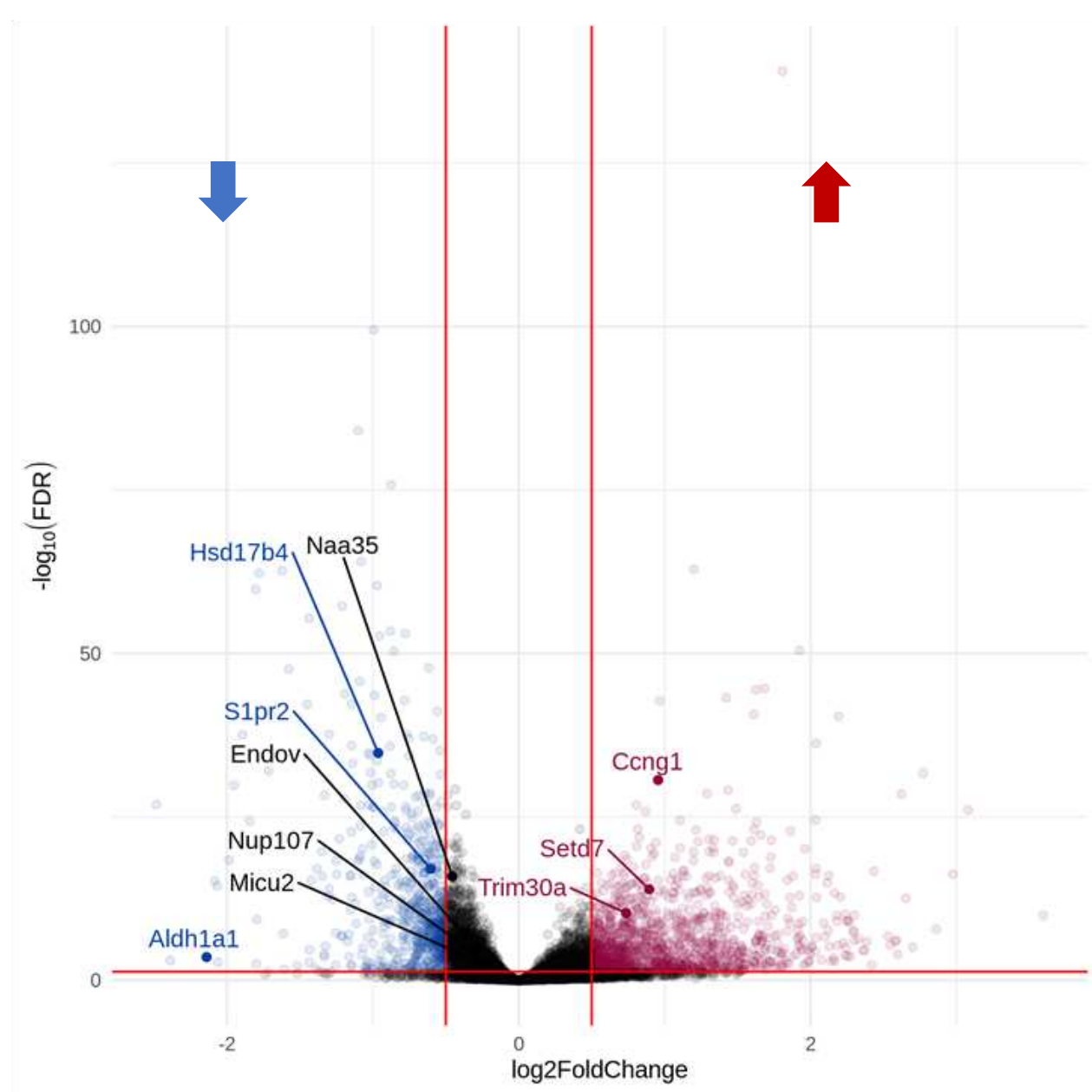

f.

\section{Quantity of tumor} Solid tumor

Digestive system cancer

Cancer Neoplasia of cells

Neoplasia of tumor cell lines

- xtracramial solin tuman

\section{g.}

\section{Epithelial neoplasm Formation of solid tumor Tumorigenesis of tissue Solid tumor \\ Non-hematological solid tumor Extracranial solid tumor Genitourinary tumor Carcinoma}


a.

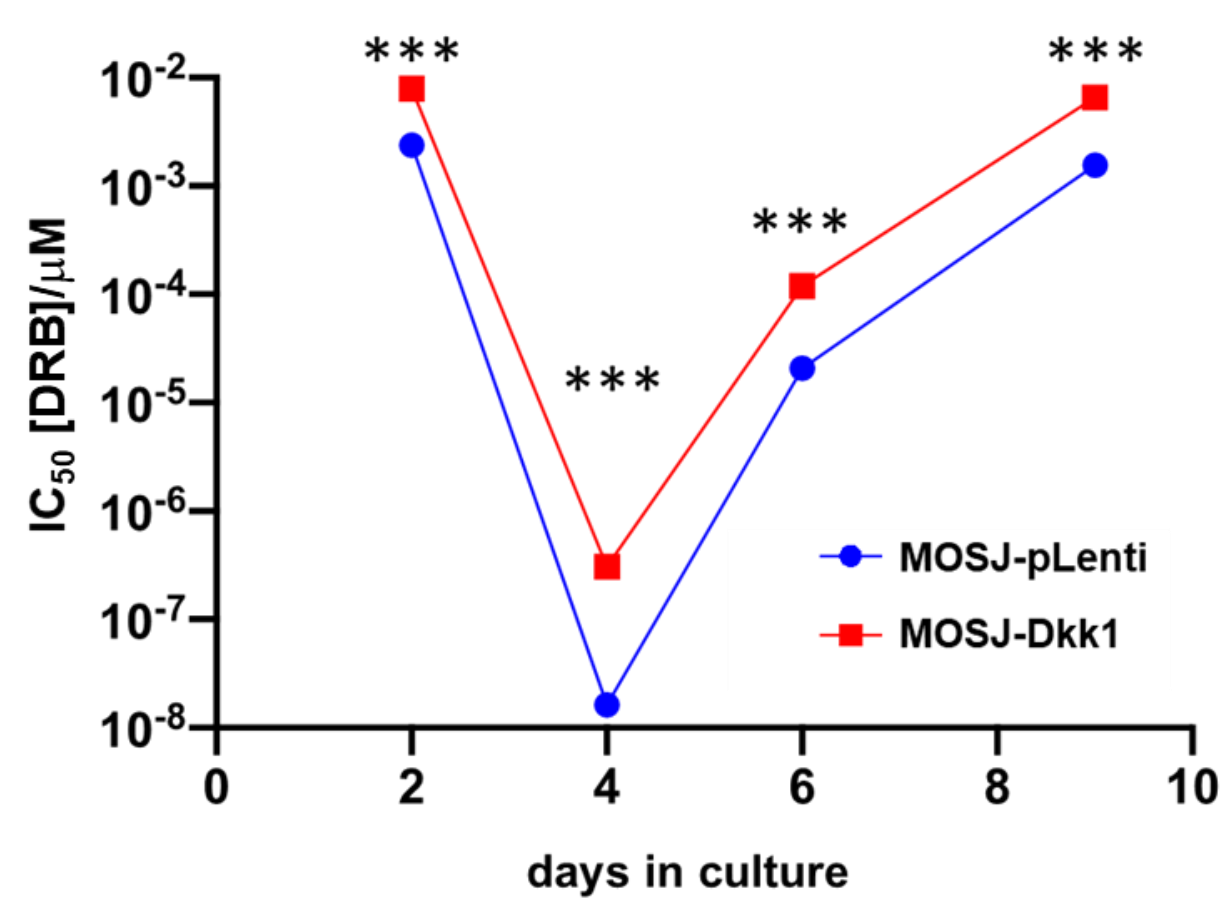

b.

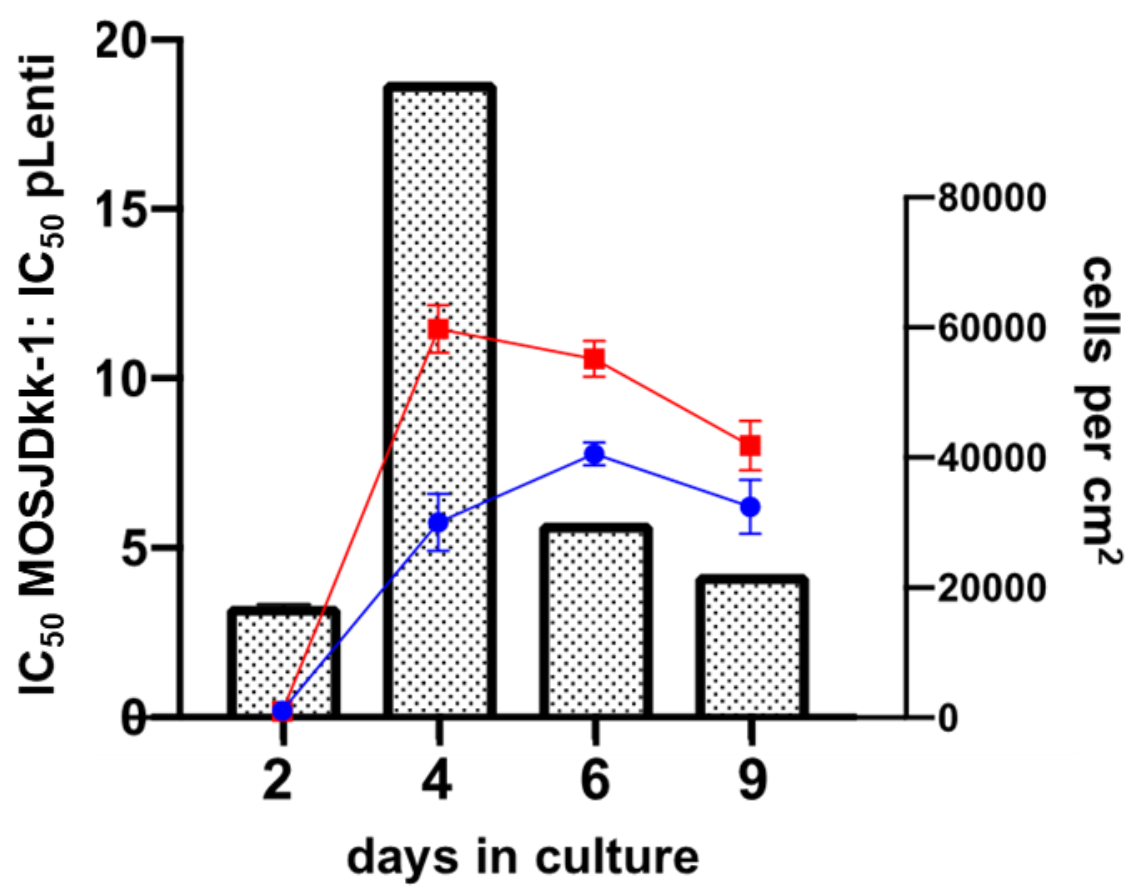

c.
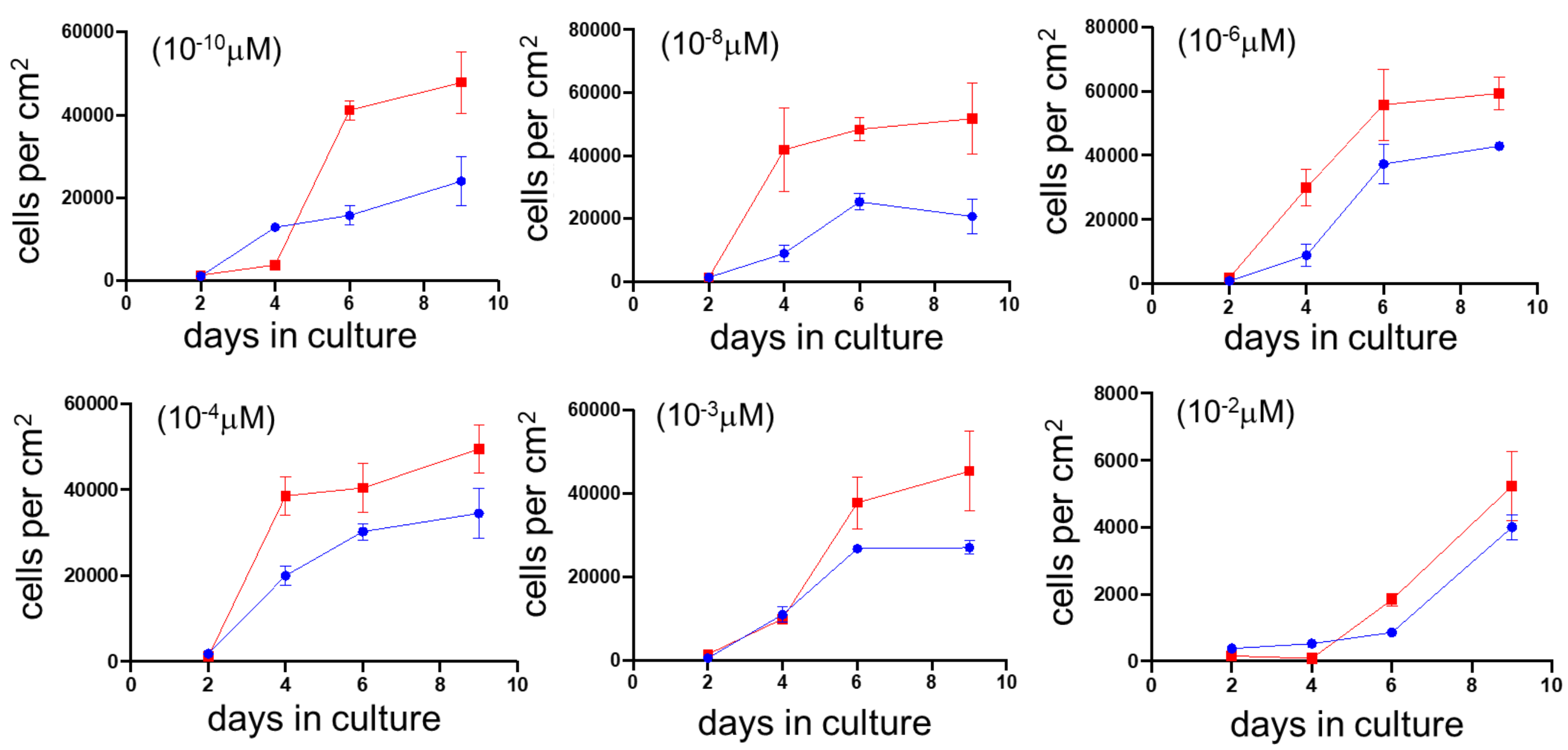

d.

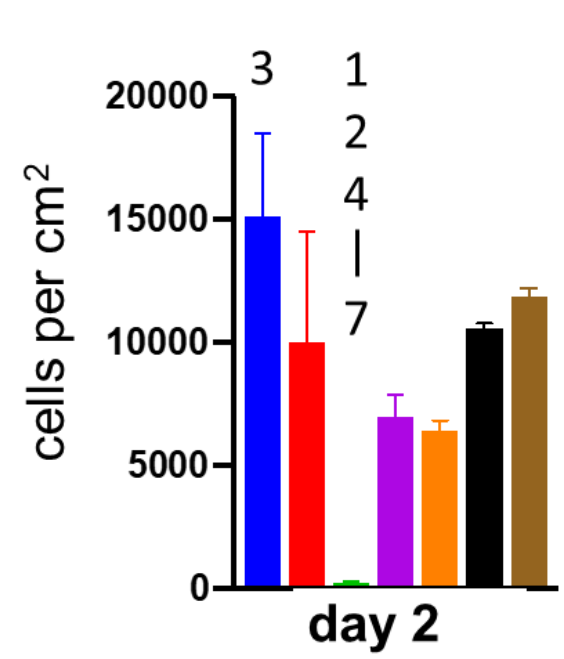

231777

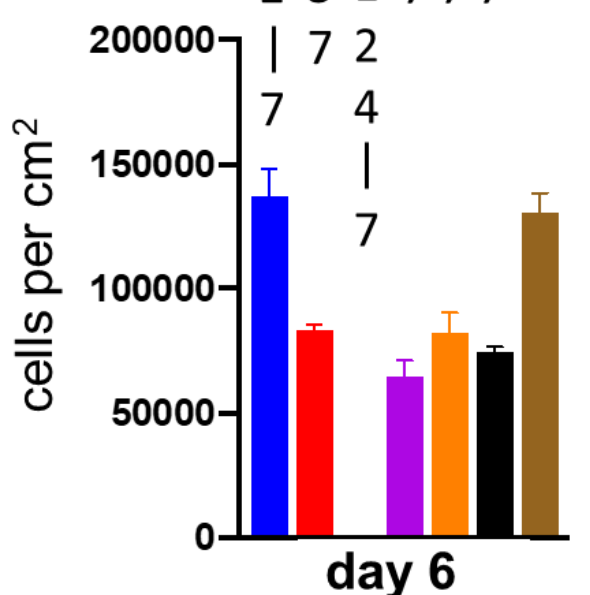

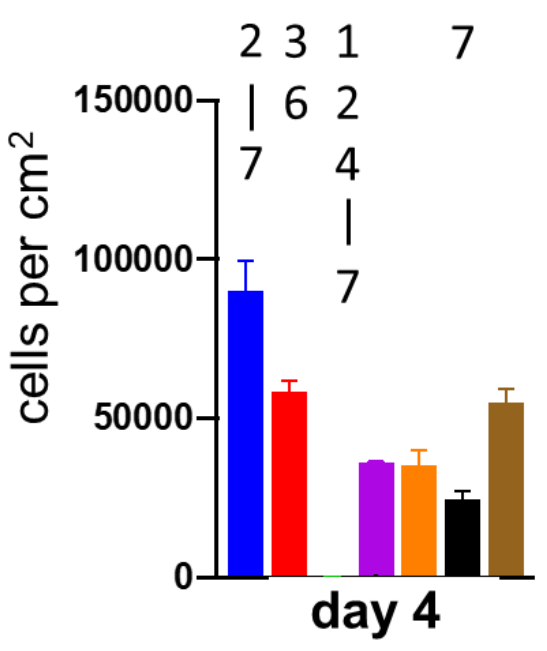

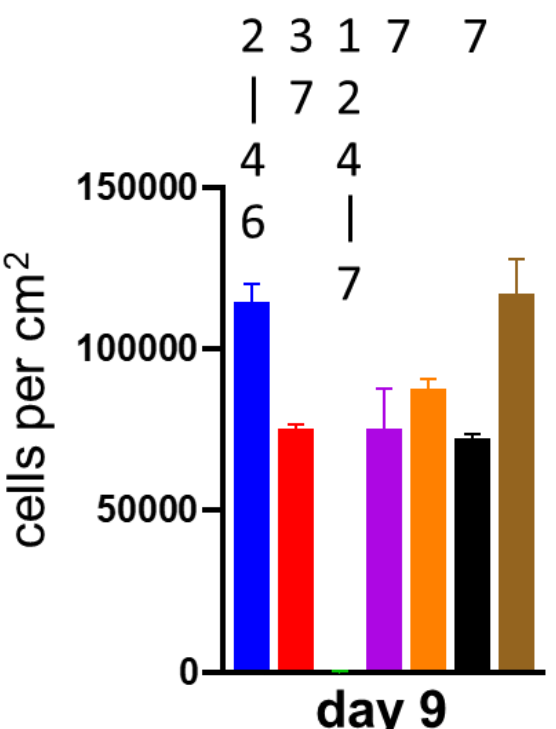

1. No treatment

2. DRB at $\mathrm{LC}_{50}$

3. DRB at $\mathrm{LC}_{50}+$ DkkMo $(5 \mu \mathrm{M})$

4. DRB at $\mathrm{LC}_{50}+$ DkkMo $(2.5 \mu \mathrm{M})$

5. DRB at $\mathrm{LC}_{50}+\operatorname{scrMo}(5 \mu \mathrm{M})$

6. DkkMo $(5 \mu \mathrm{M})$

7. $\operatorname{scrMo}(5 \mu \mathrm{M})$ 
a.

No treatment DRB 5 mg.kg-1 DkkMo 12.5 mg.kg-1 Combination
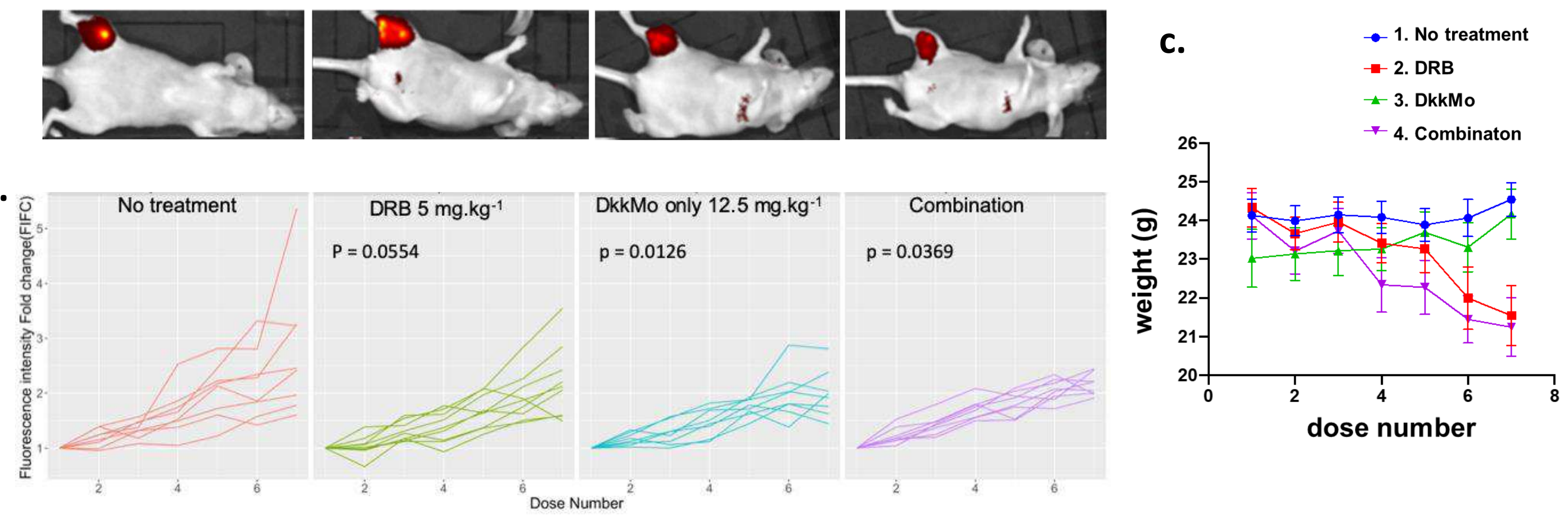

d.
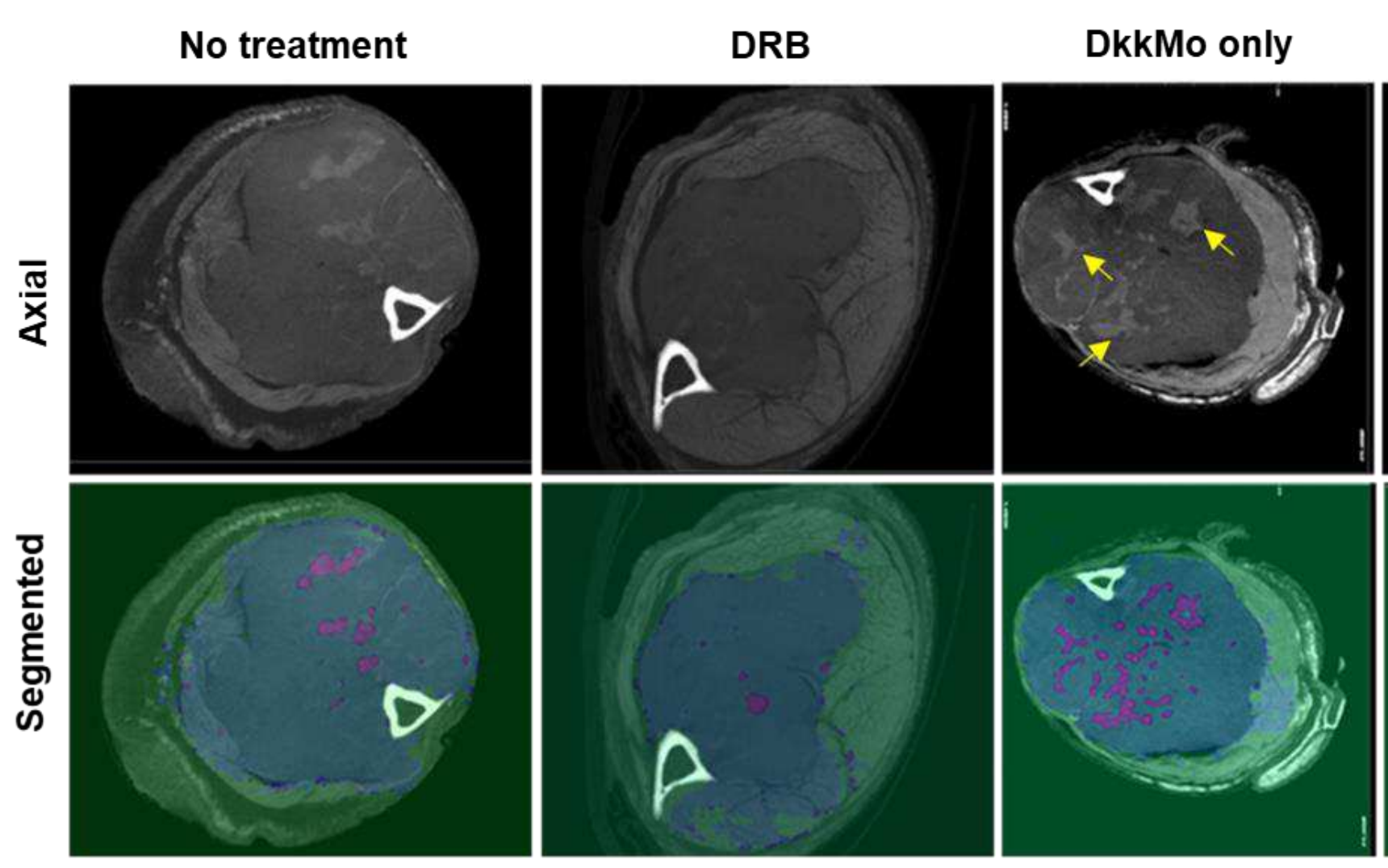

\section{Combination}
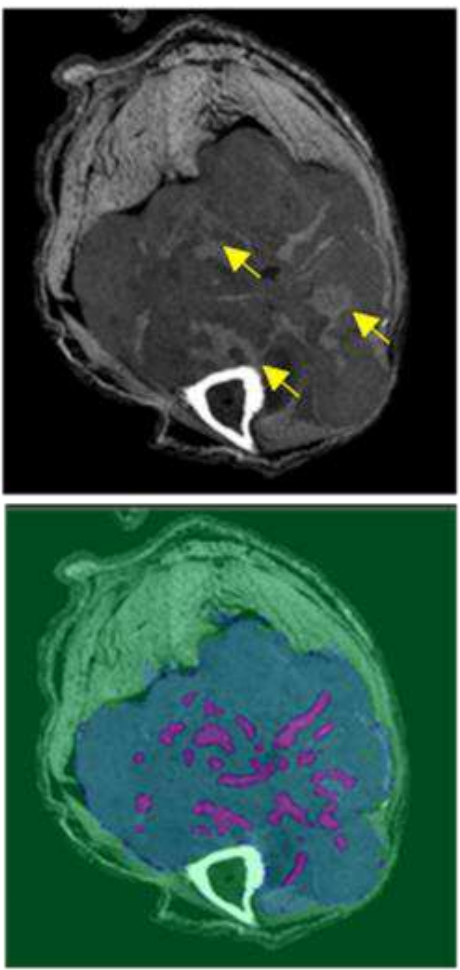

f.

e.
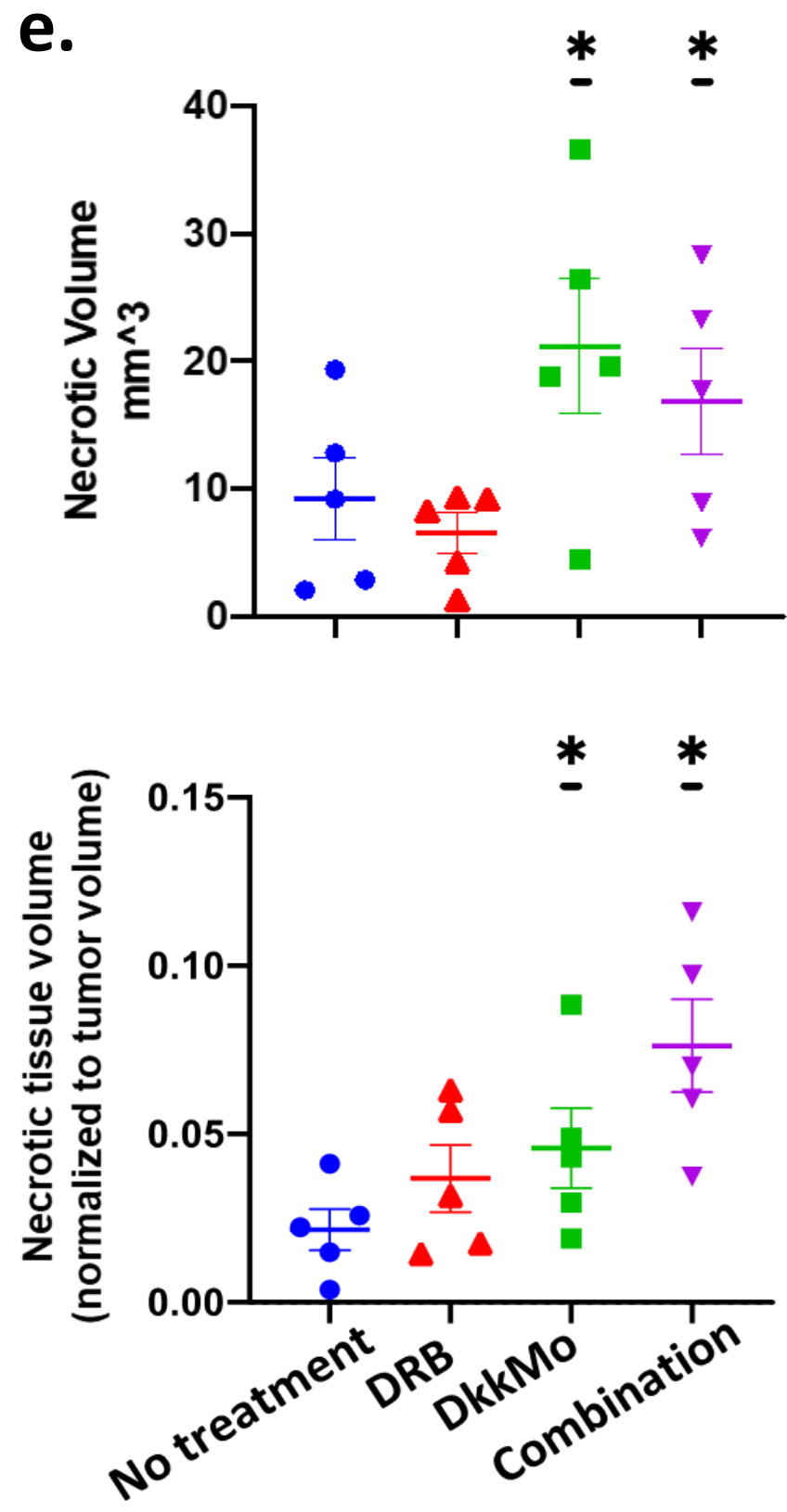

No treatment DRB 5 mg. $\mathbf{k g}^{-1}$ DkkMo 12.5 mg.kg-1

Combination
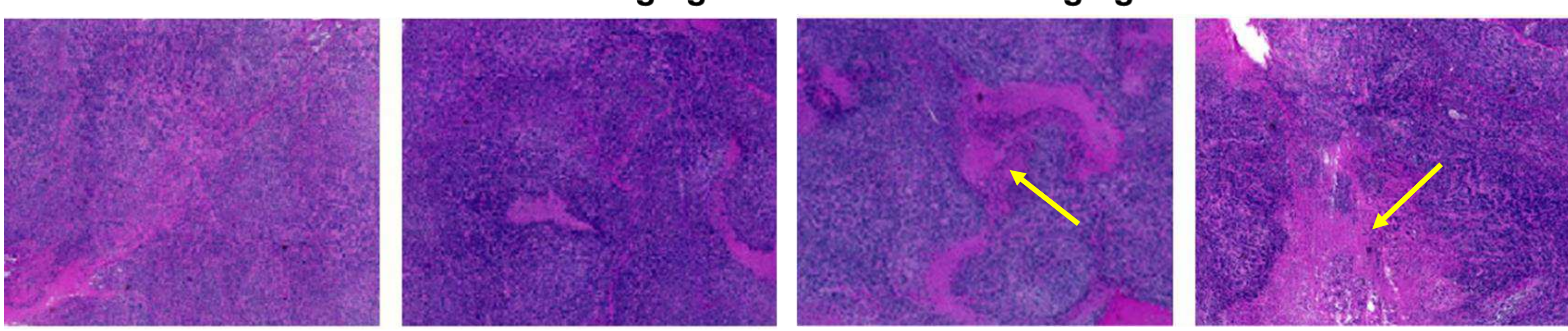


\section{Figure 5.}

a.

Healthy bone (equivalence to contralateral).

surface voxels of

sample bone

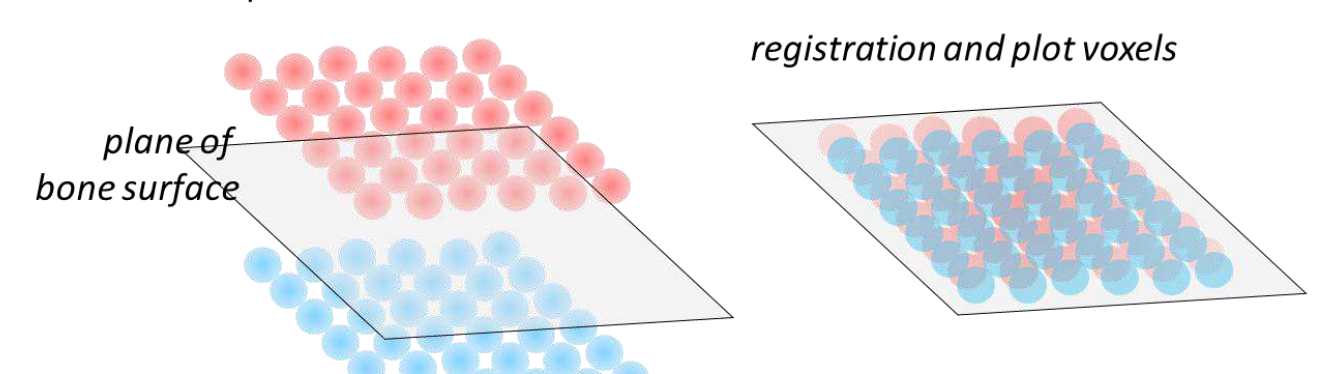

plot deviation of coordinates

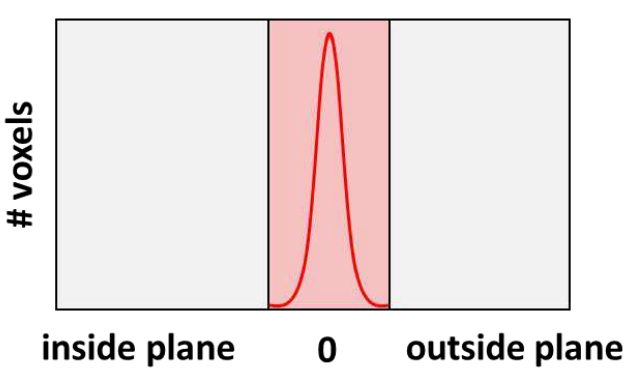

surface voxels of contralateral bone mirrorimage

\section{Remodeled bone (deviation from contralateral).}

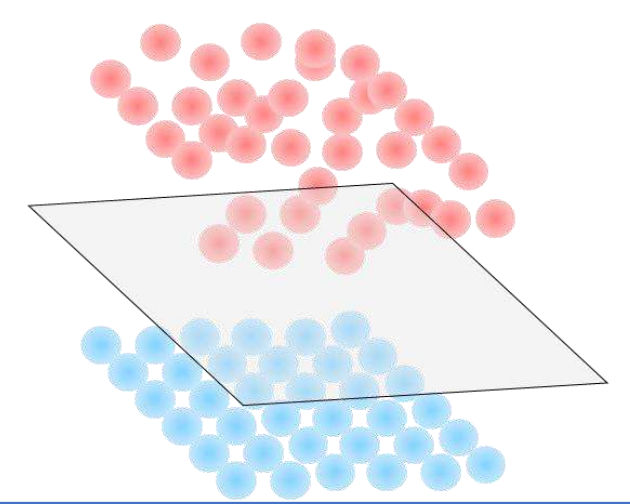

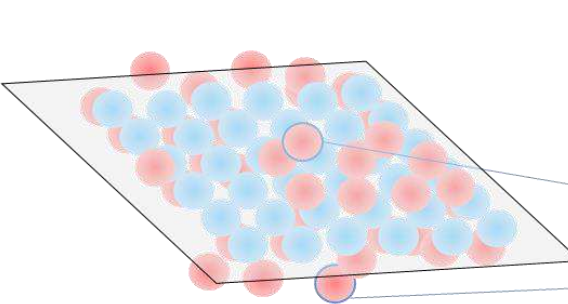

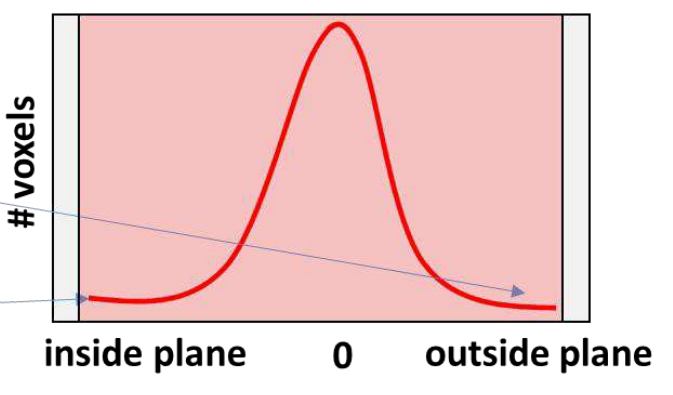

b.

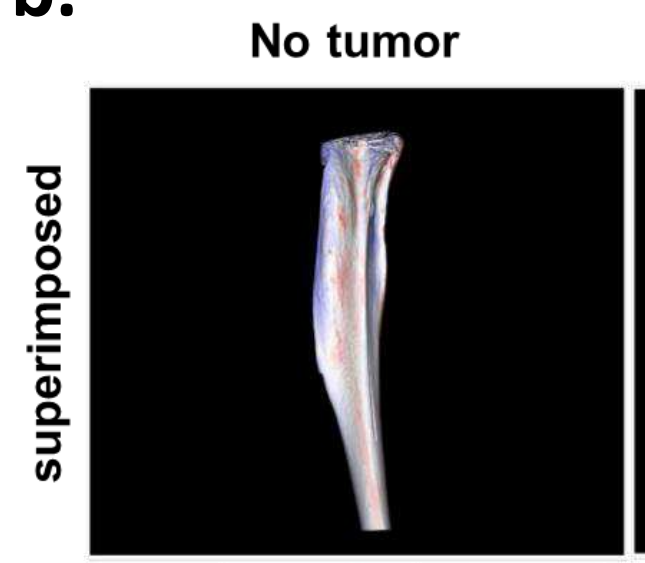

No treatment

DRB 5 mg. . $^{-1}$

DkkMo only 12.5 mg.kg-1

Combination
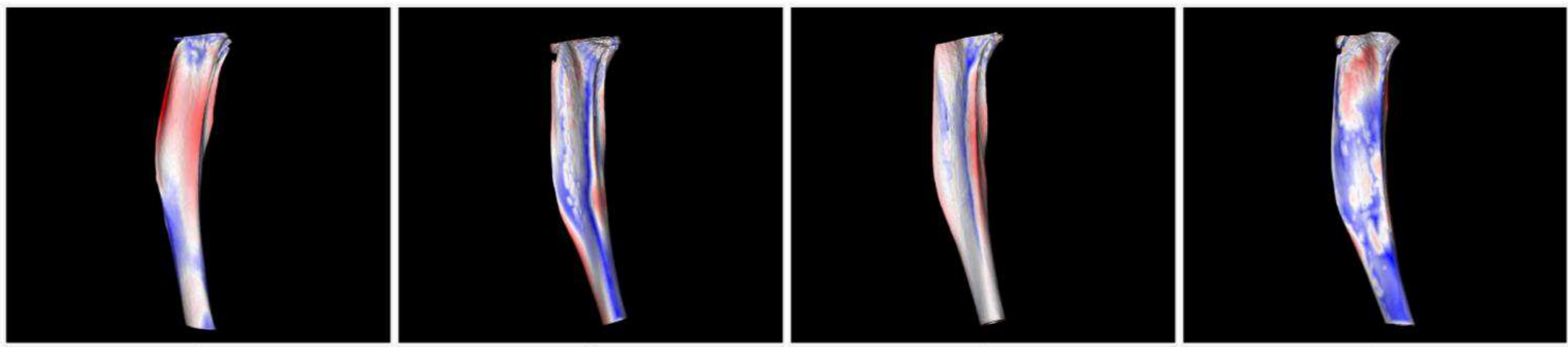

c.
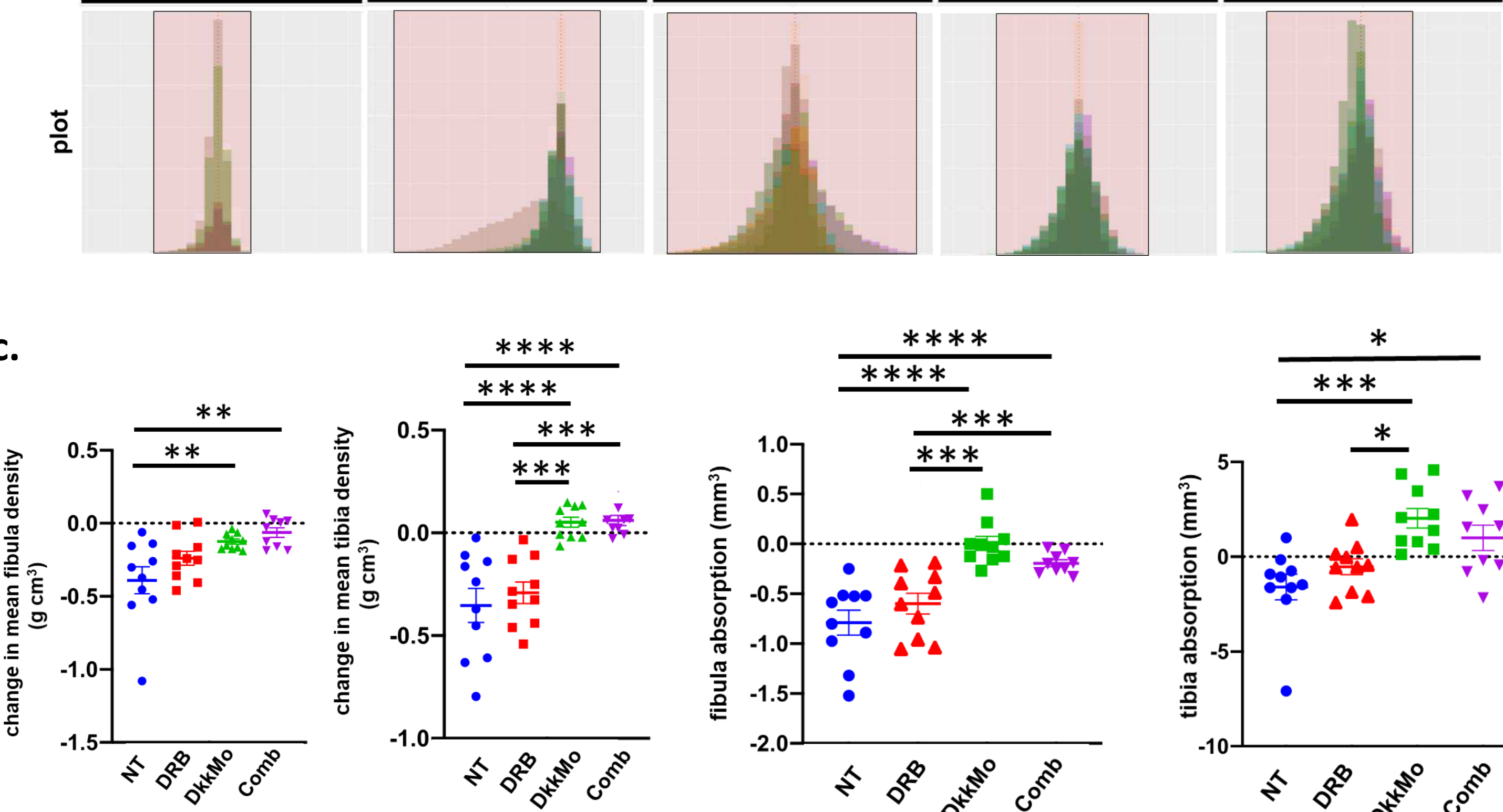

\section{흥}
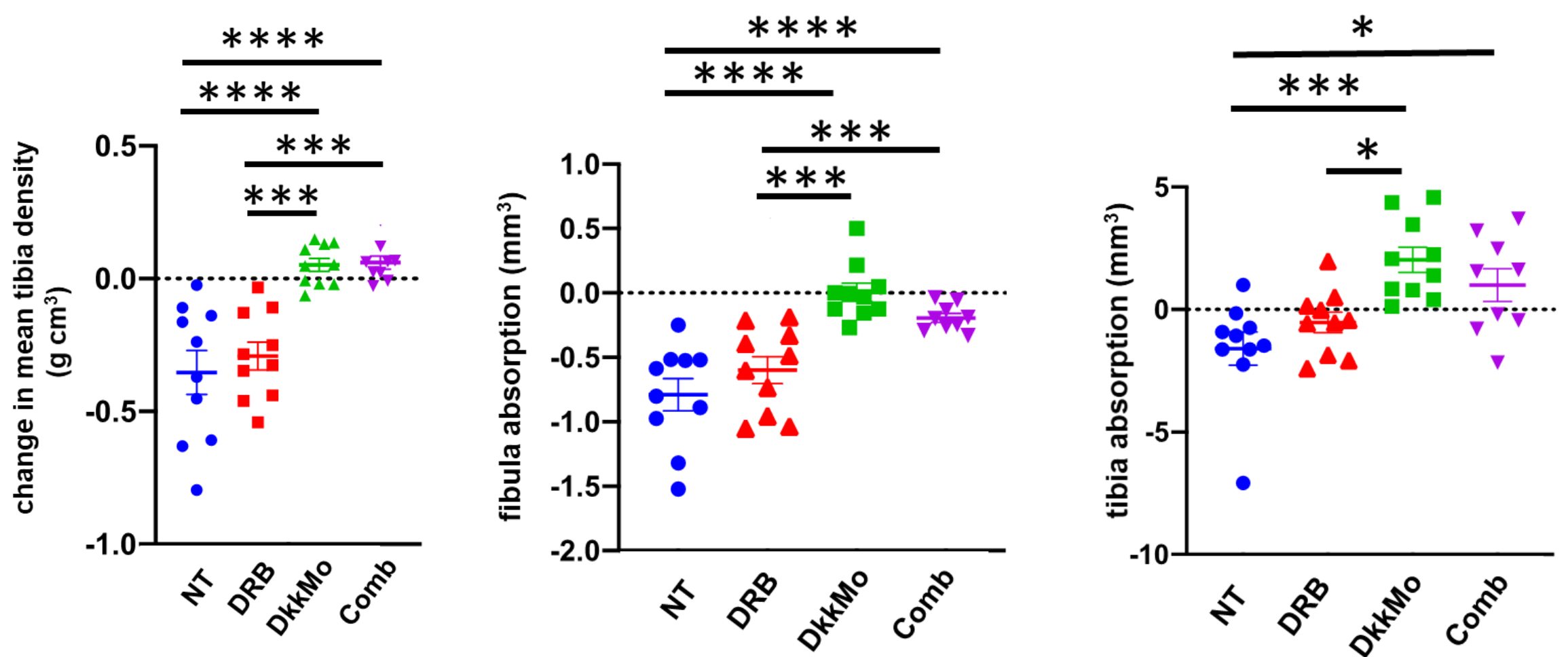

d.
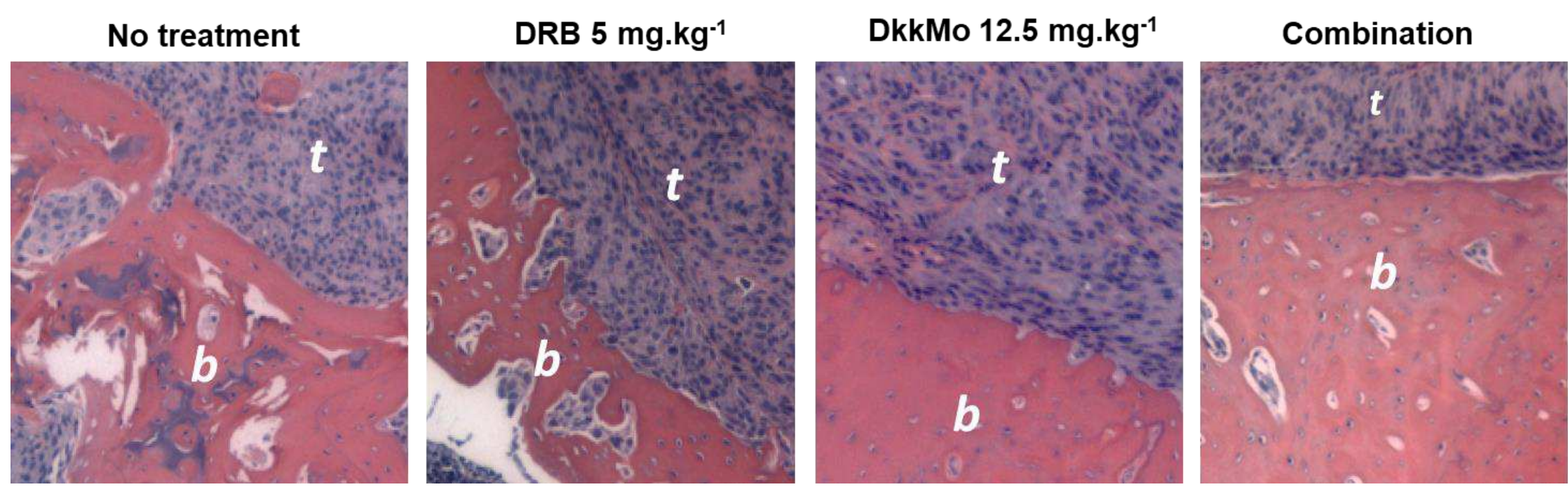


\section{Figure 6.}

a.

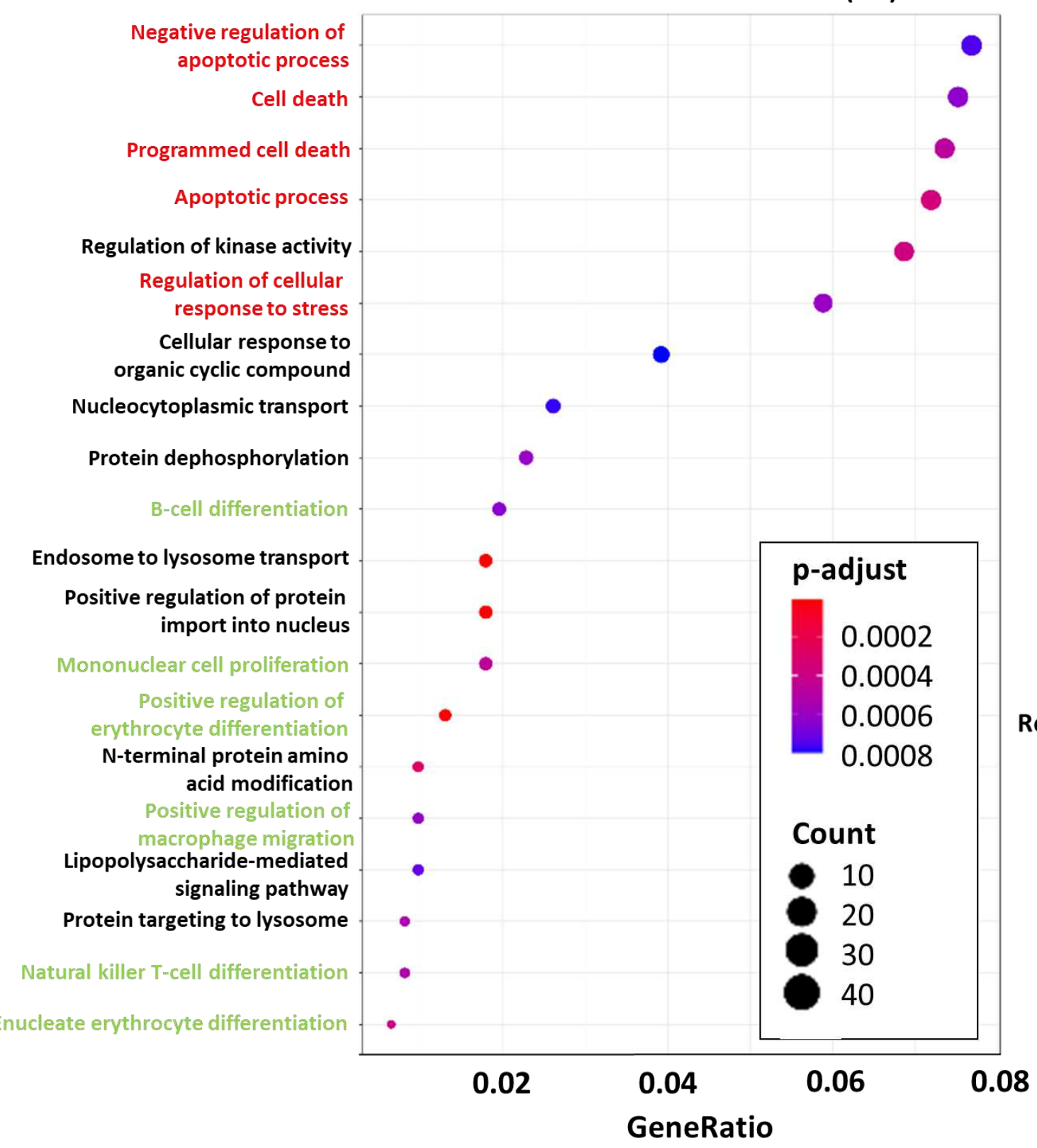

C.

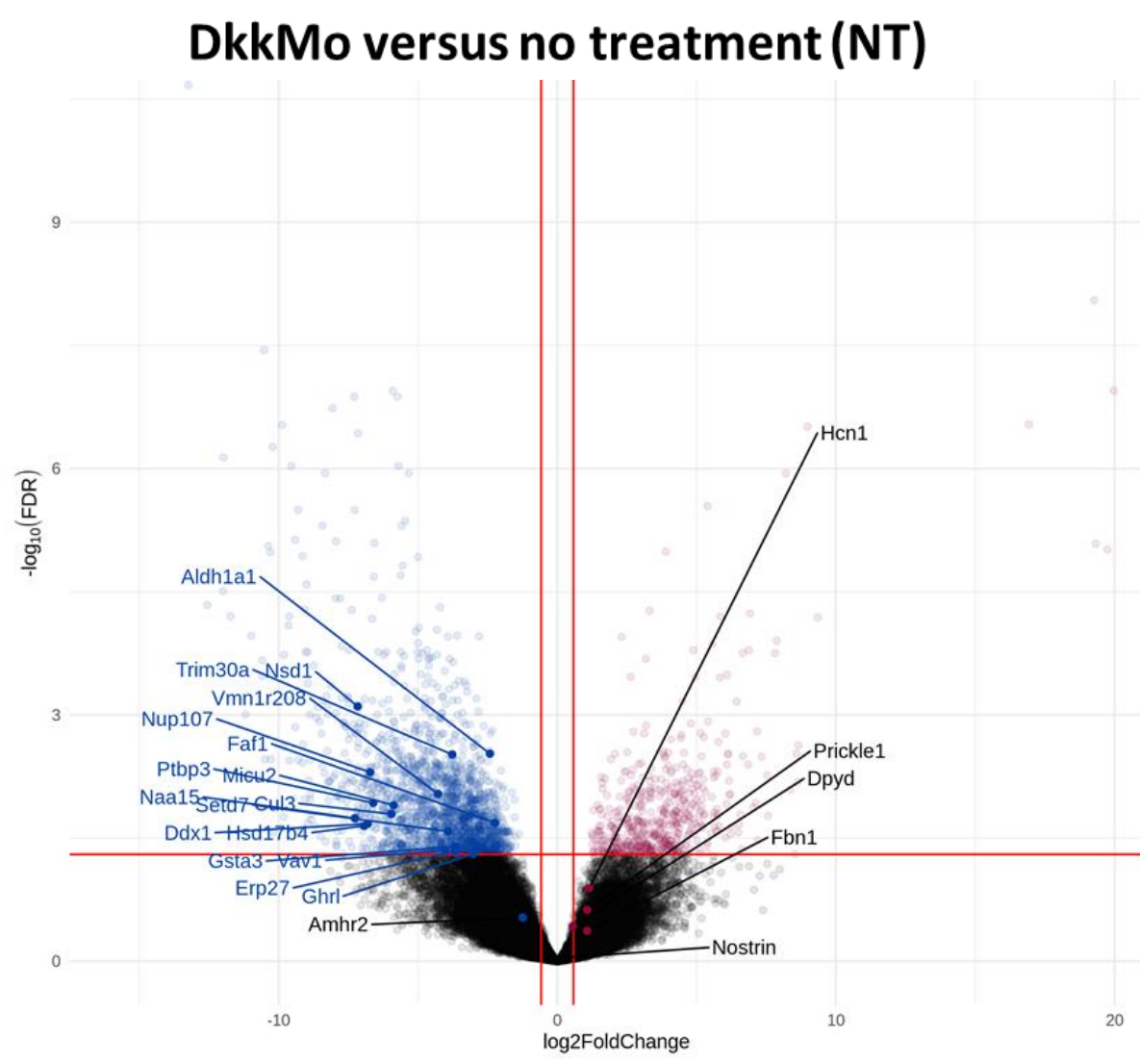

d.

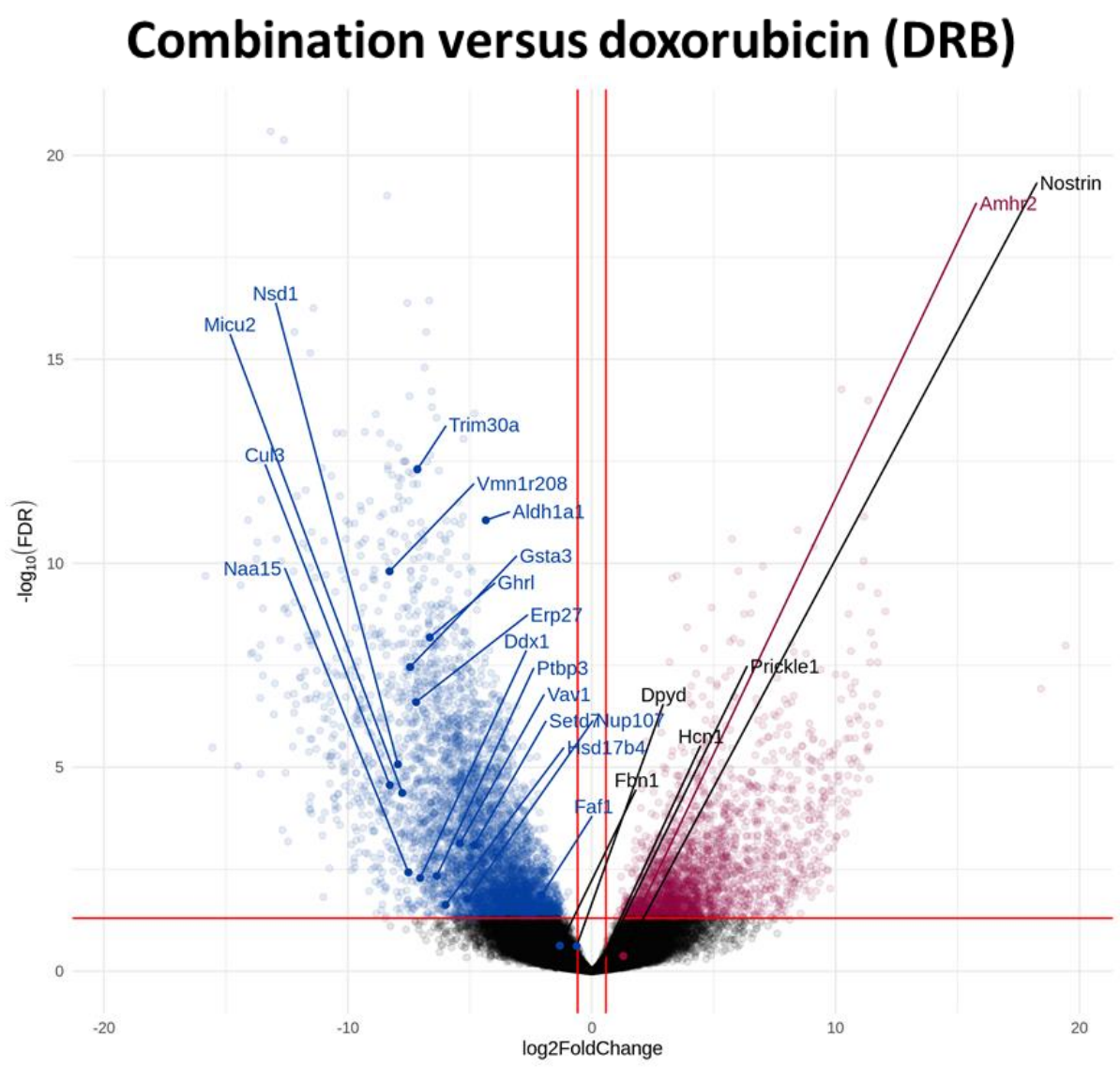

b.

Combination versus doxorubicin (DRB)

Negative regulation of nitrogen compound metabolic process Negative regulation of response to stimulus

Cell death

Programmed cell death

Apoptotic Process

Regulation of kinase activity

Protein phosphorylation

G-protein coupled receptor signaling pathway

Positive regulation of kinase activity

Transmembrane receptor tyrosine kinase signaling pathway

Regulation of Ras protein signal transduction

Exocytosis

Positive regulation of GTPase activity

Positive regulation of

canonical Wnt signaling activity Steroid hormone mediated signaling pathway

$$
0.04 \quad 0.08
$$$$
\text { p-adjust }
$$

0.0002

0.0004

0.0006

e.

DkkMo versus no treatment (NT)

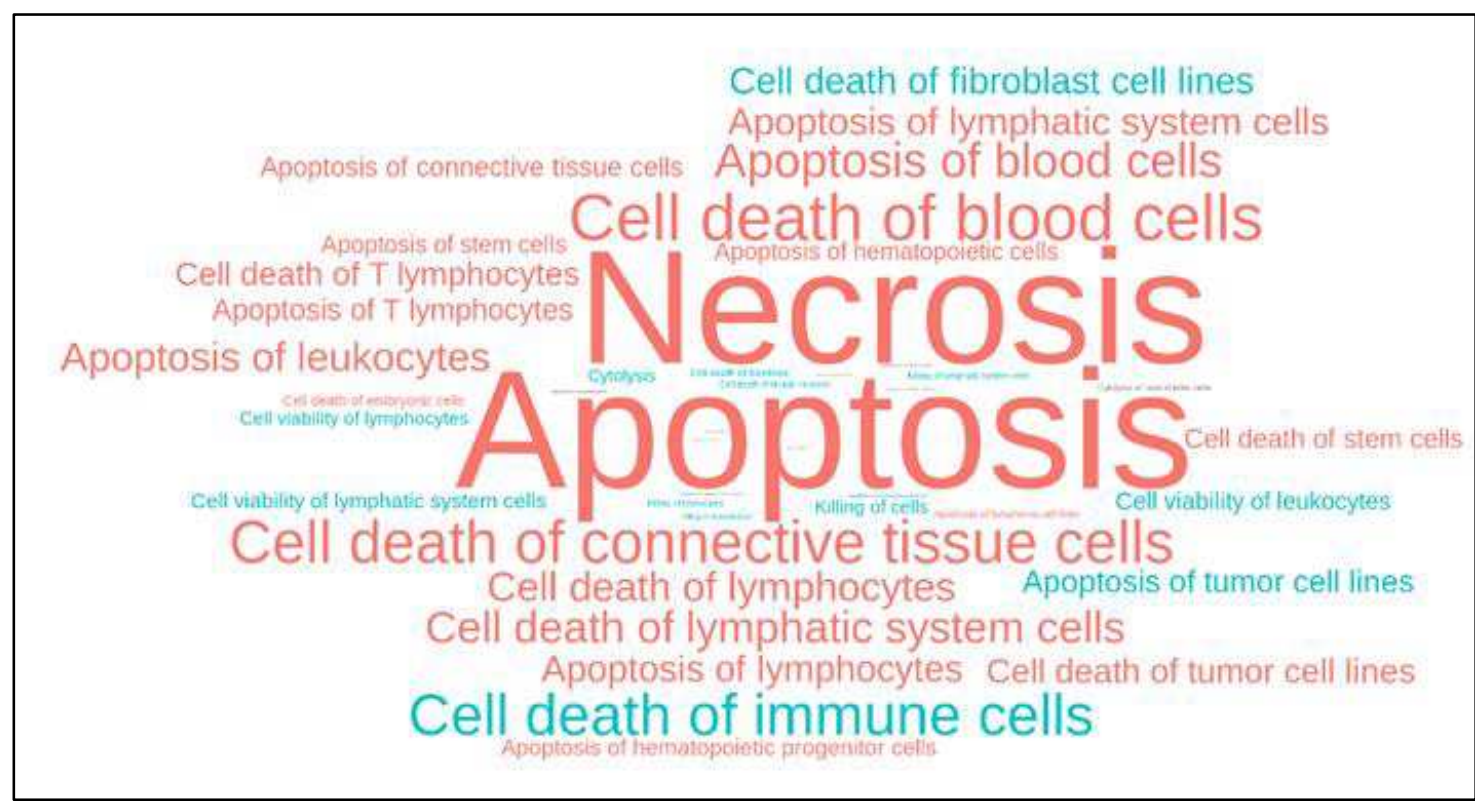

f.

Combination versus doxorubicin (DRB)

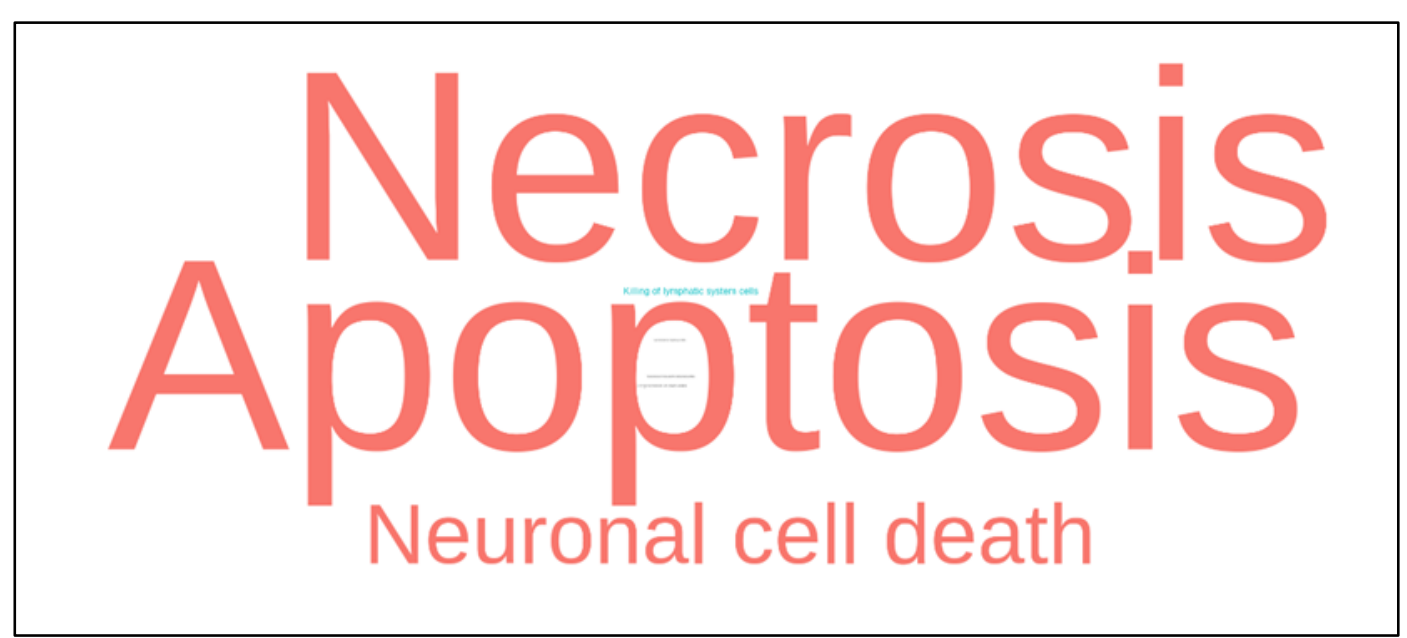

g.

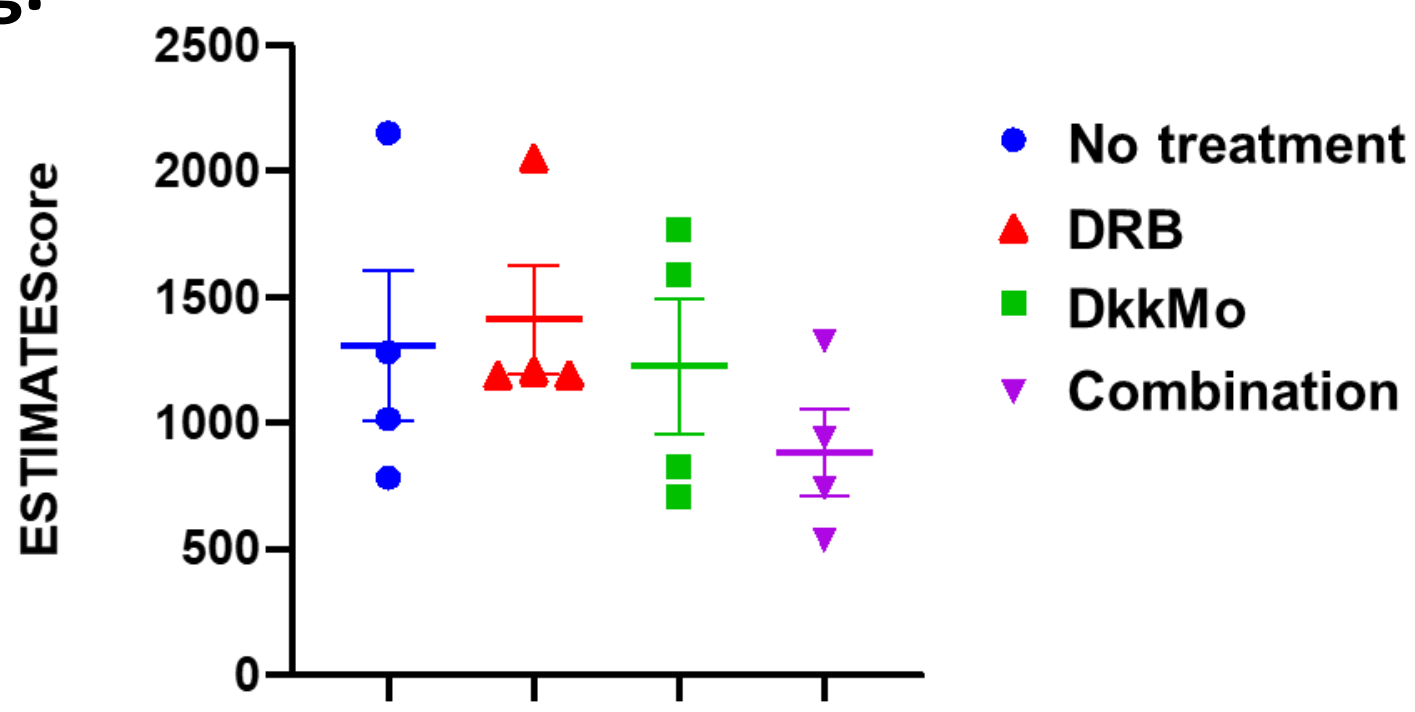




\section{Supplemental Figure 1.}

a.

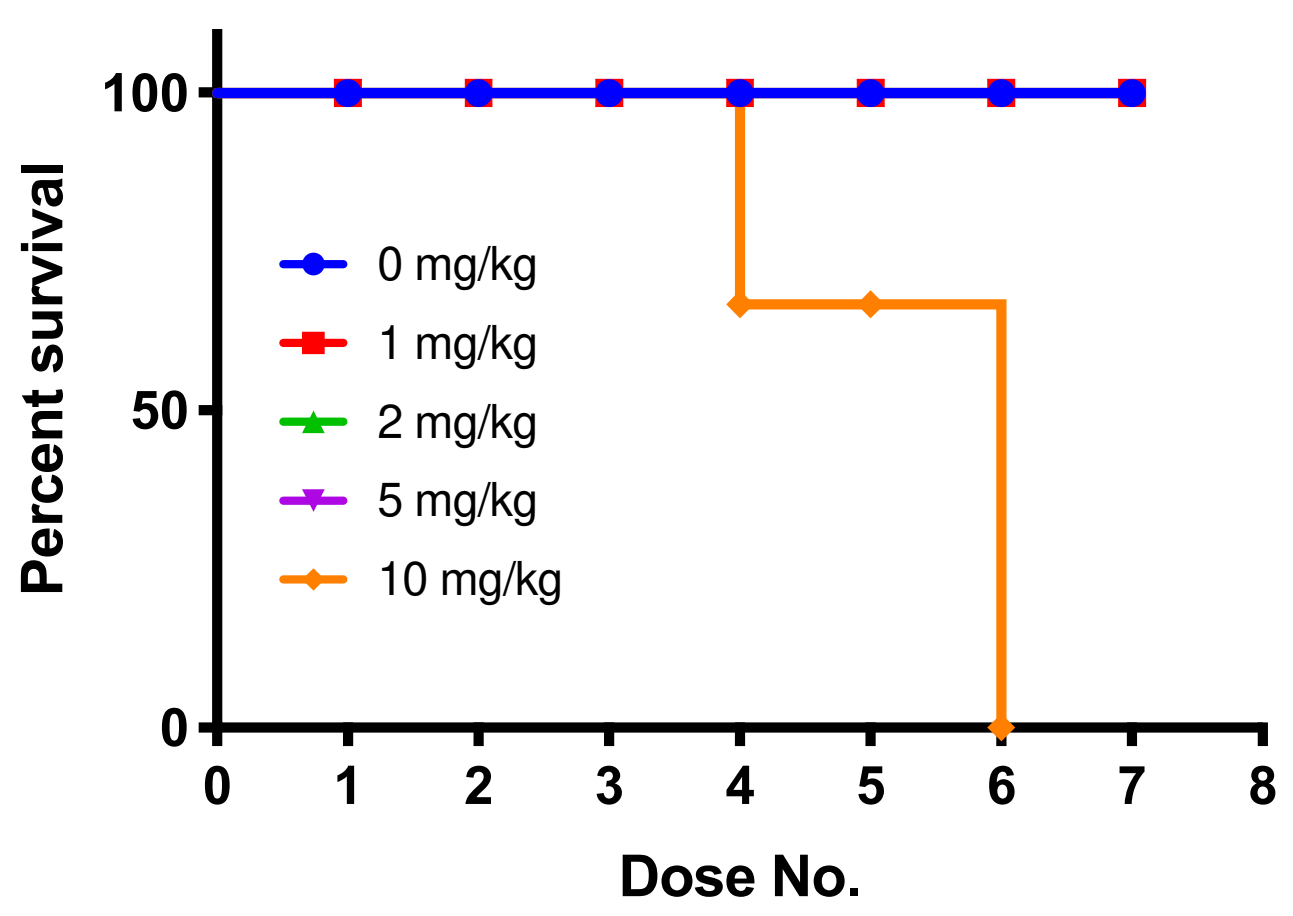

b.

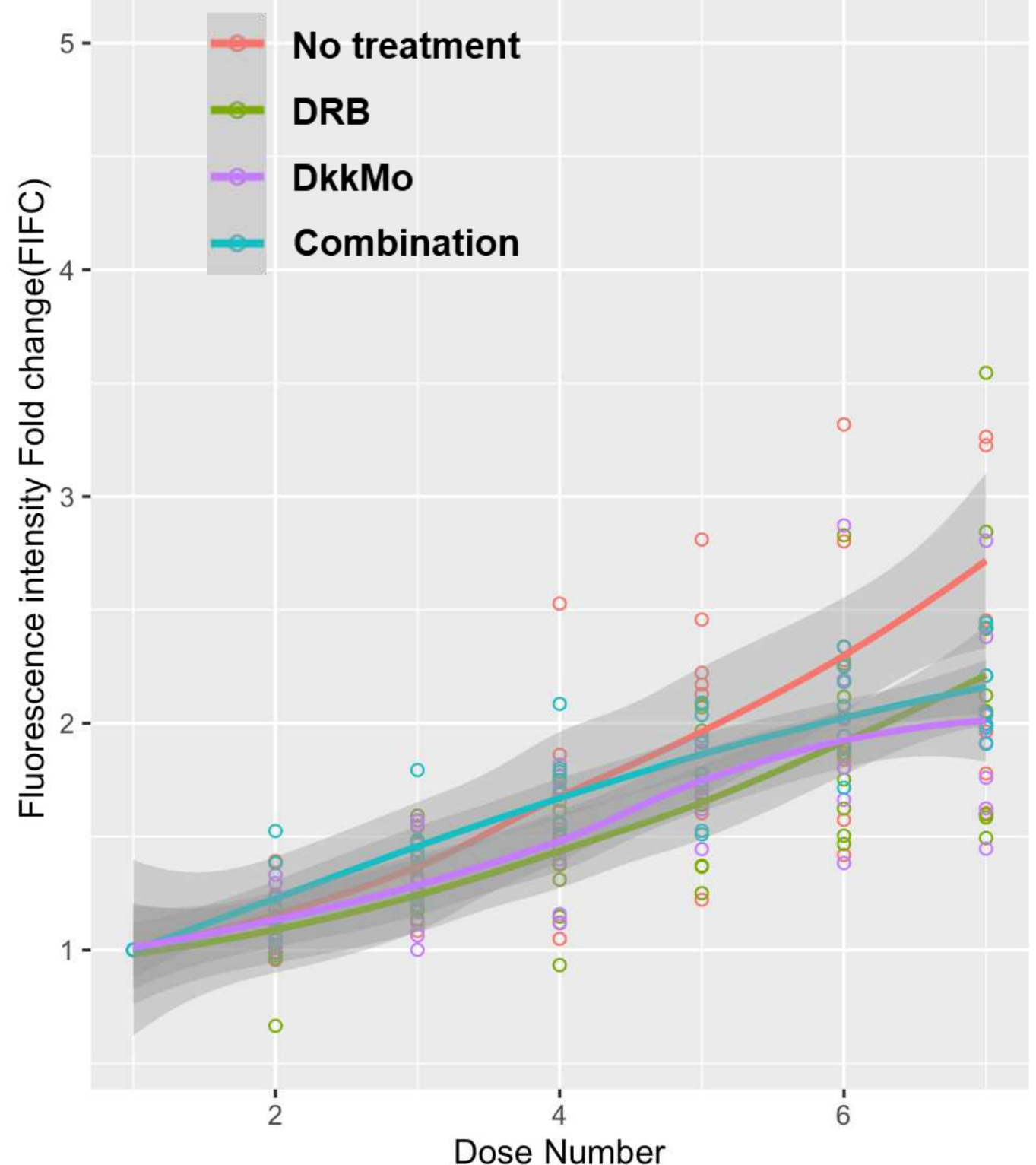

c.

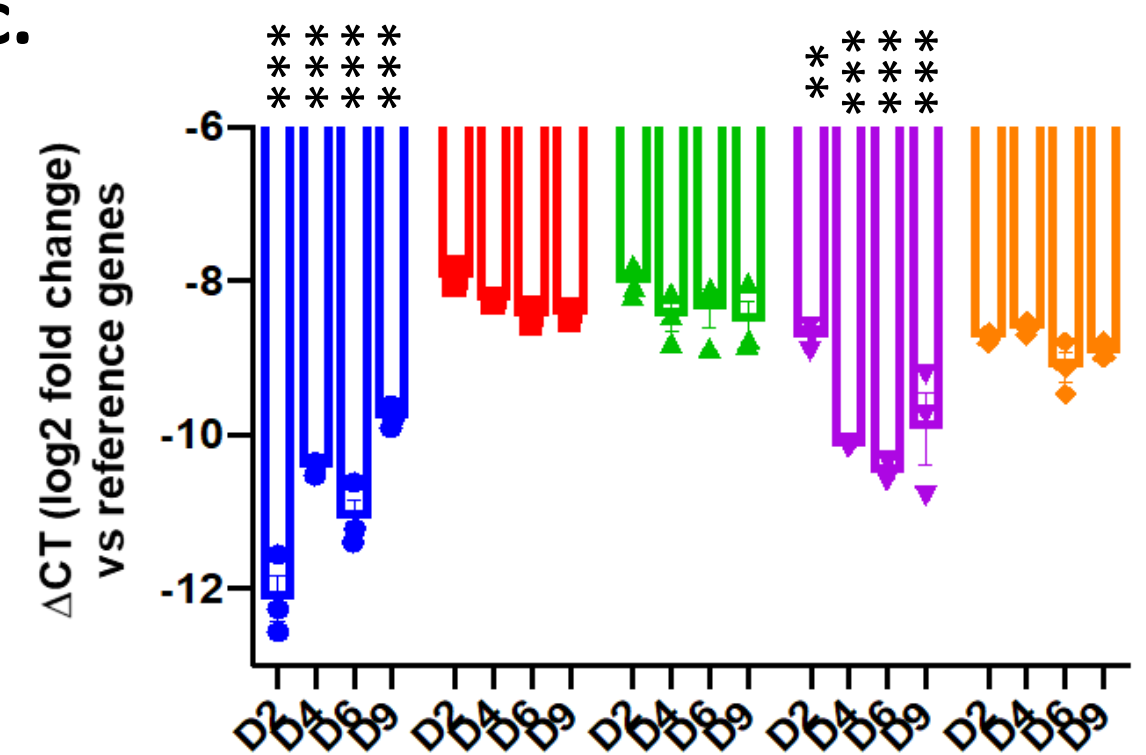

d.

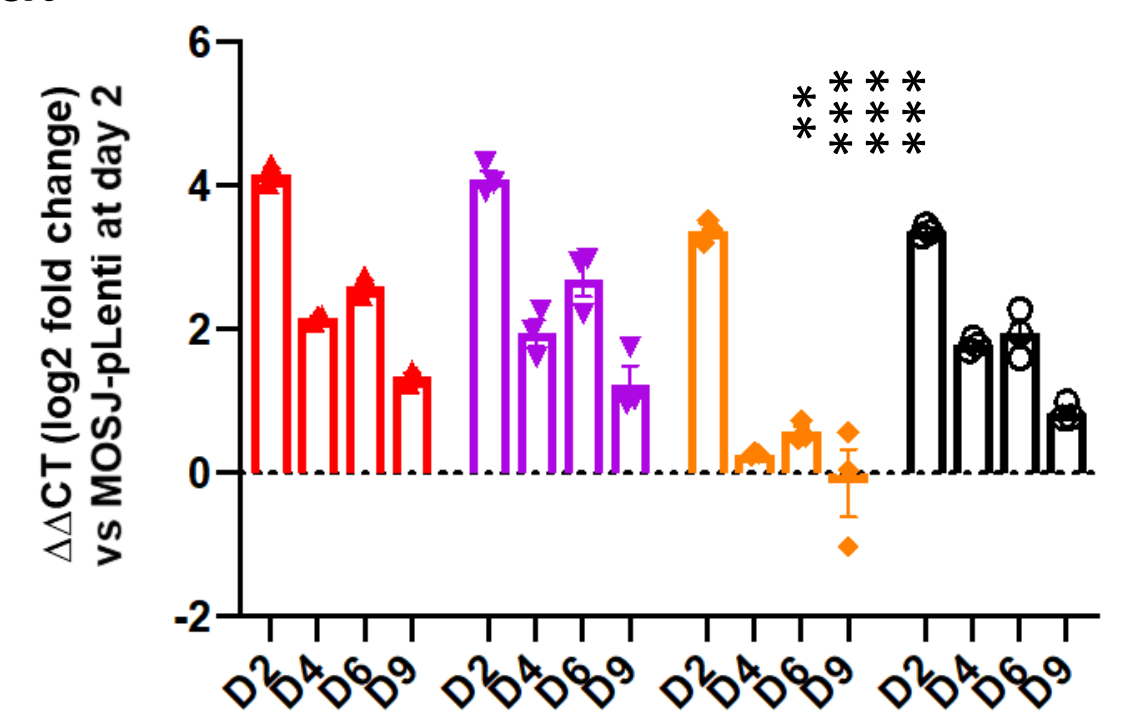

f.

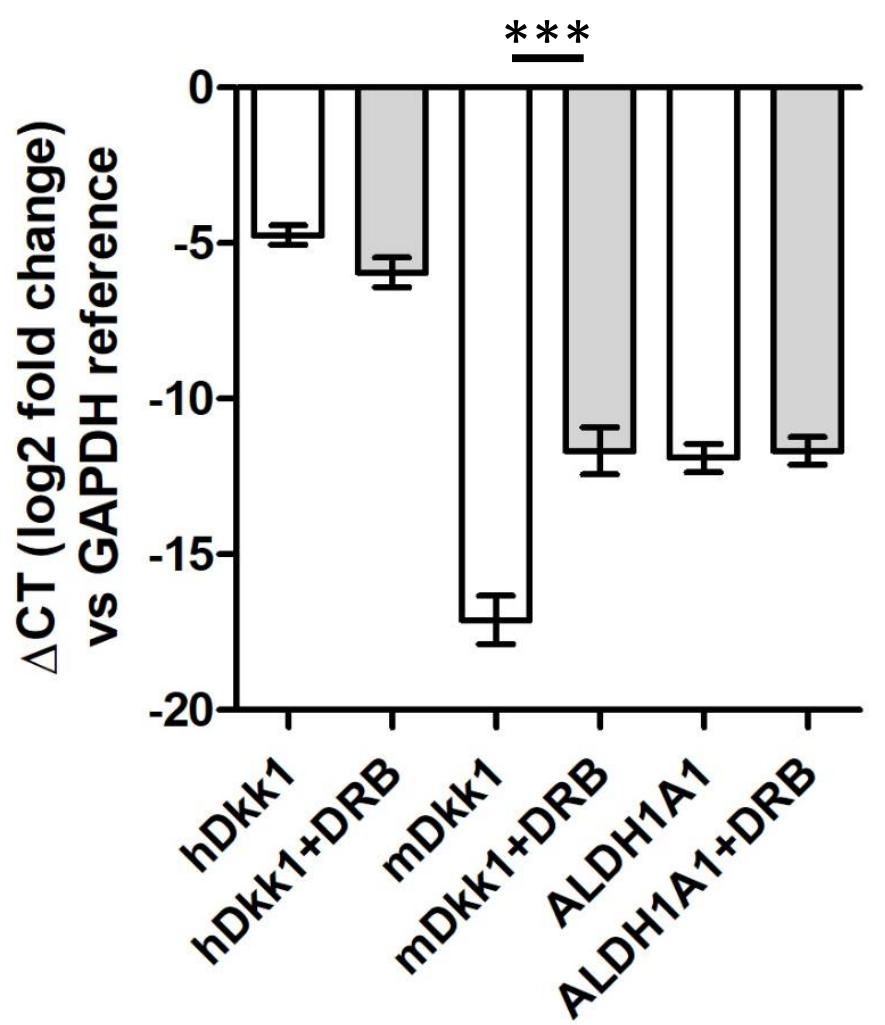




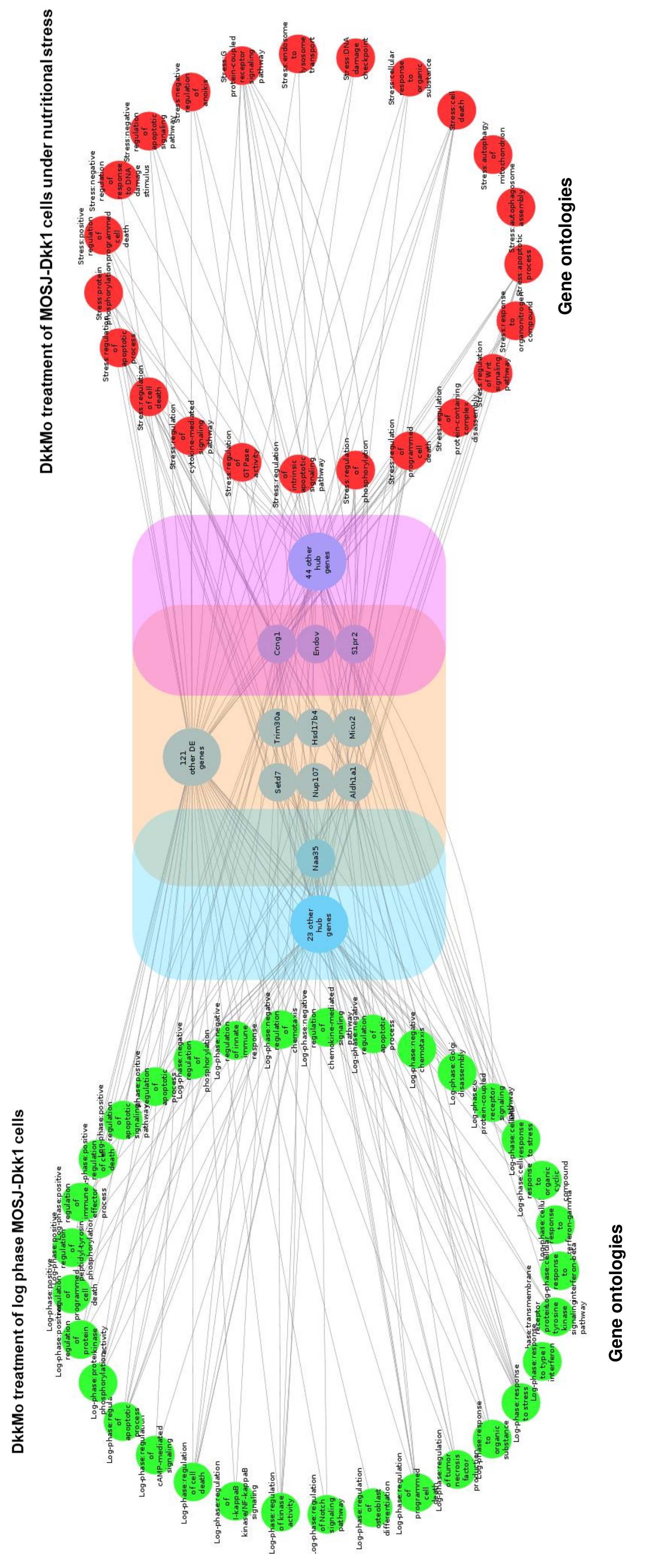




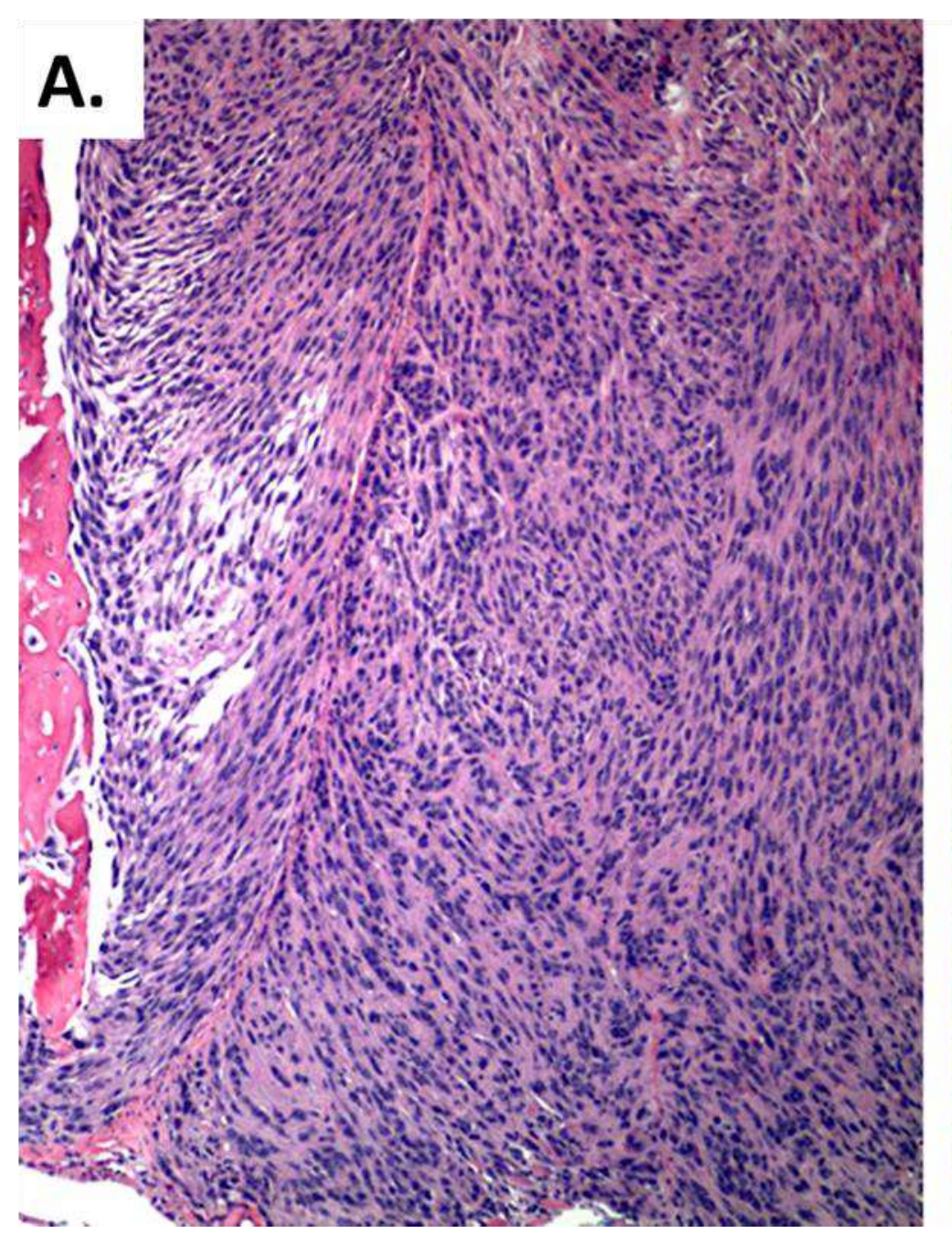

B. 3 Gow Ges. 13 s.

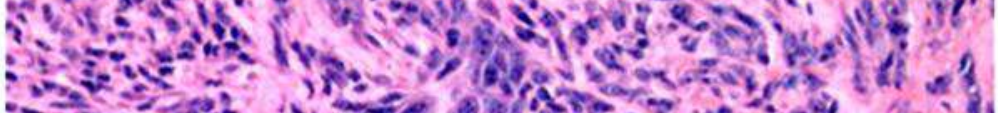

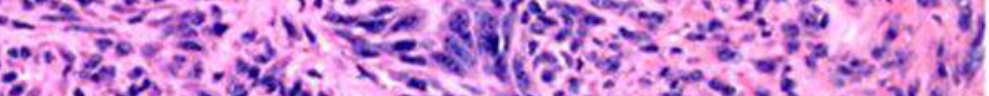

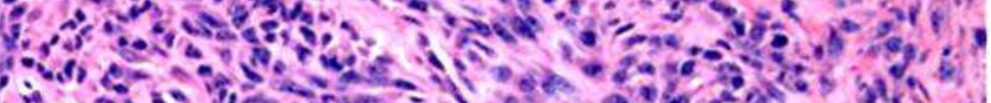

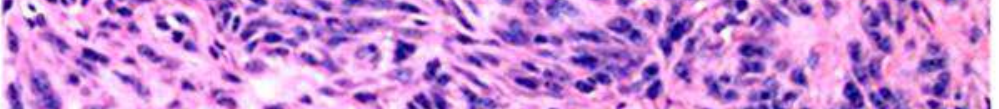
$14-\ldots 4$ s

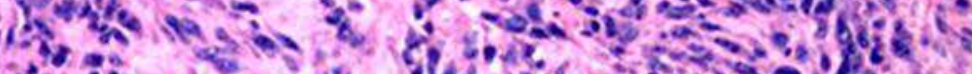

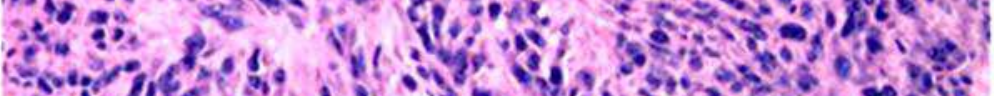

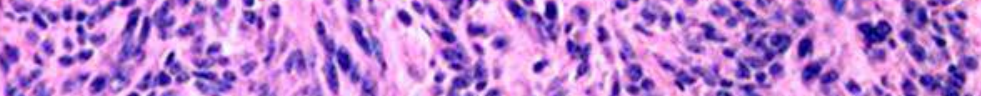

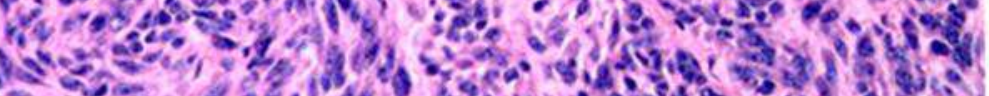

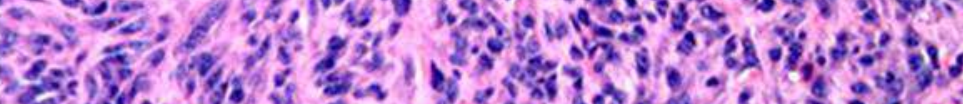

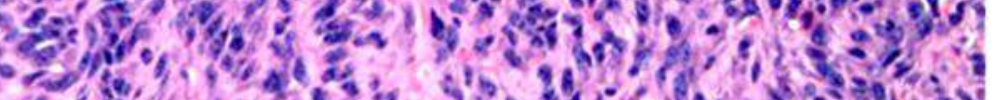

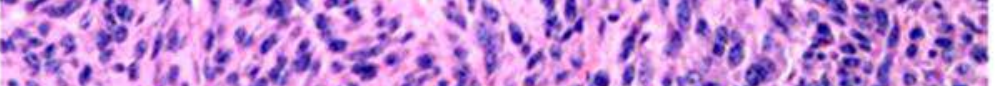

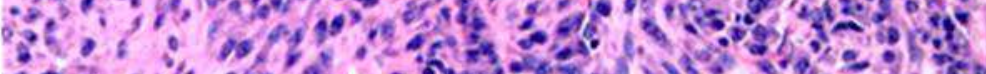

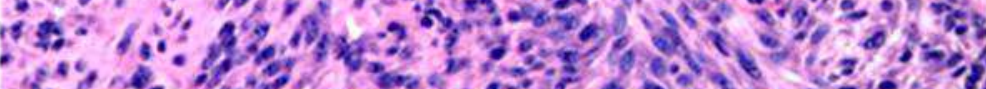

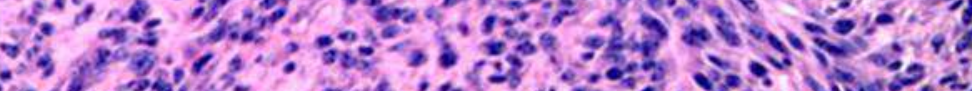

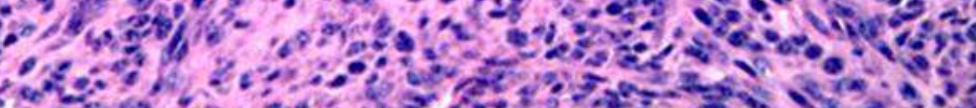

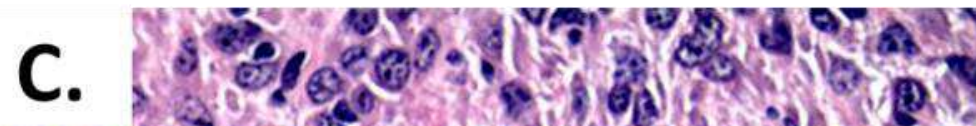

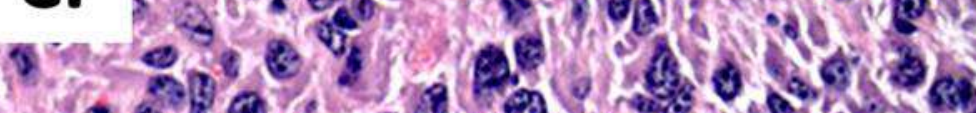

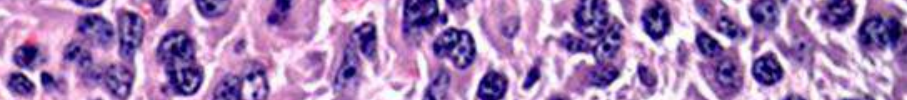

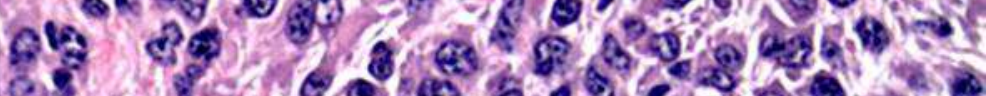

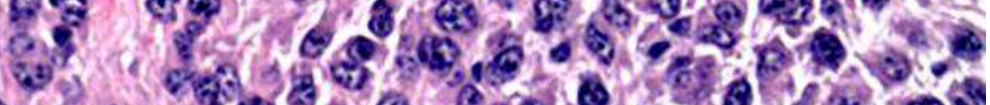

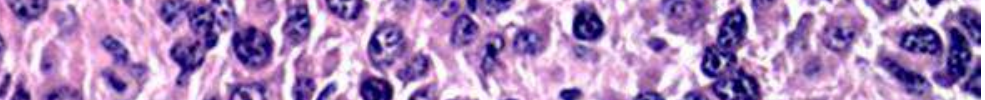

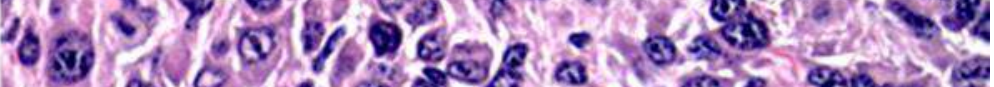
- 250.025

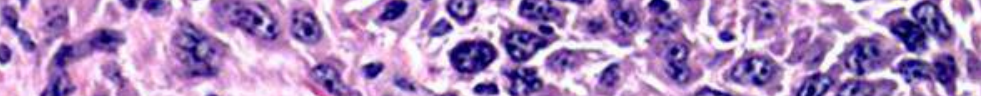
3 $0^{2}$ -

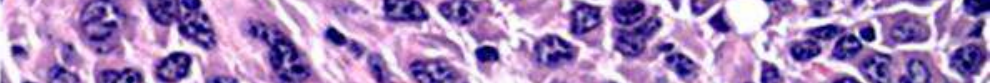

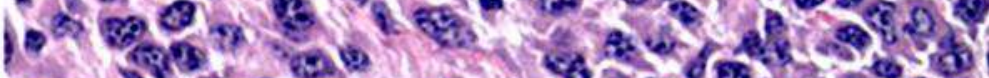

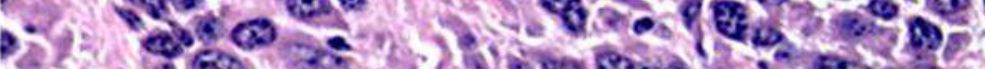

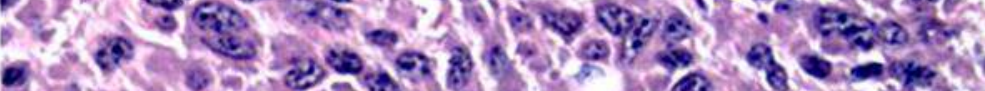

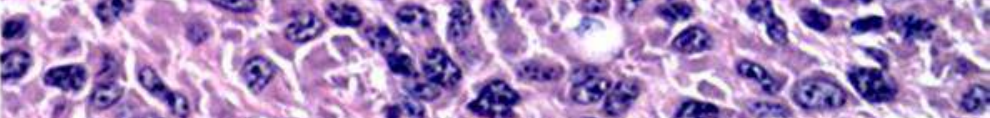

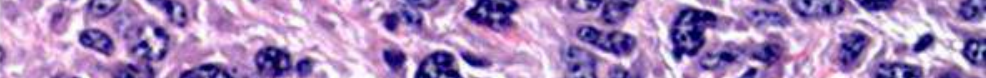

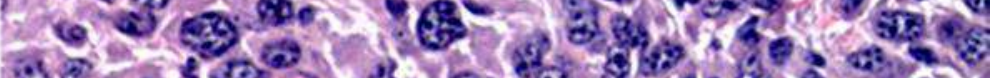

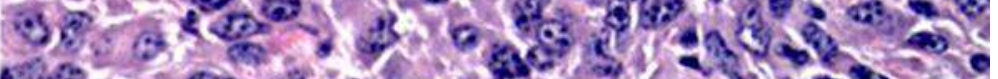

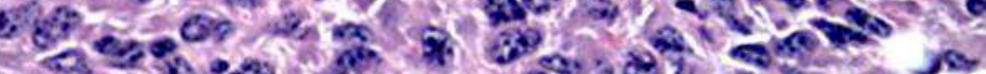

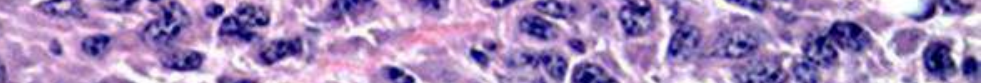

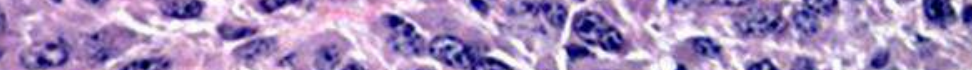
\%2. ${ }^{2}$.
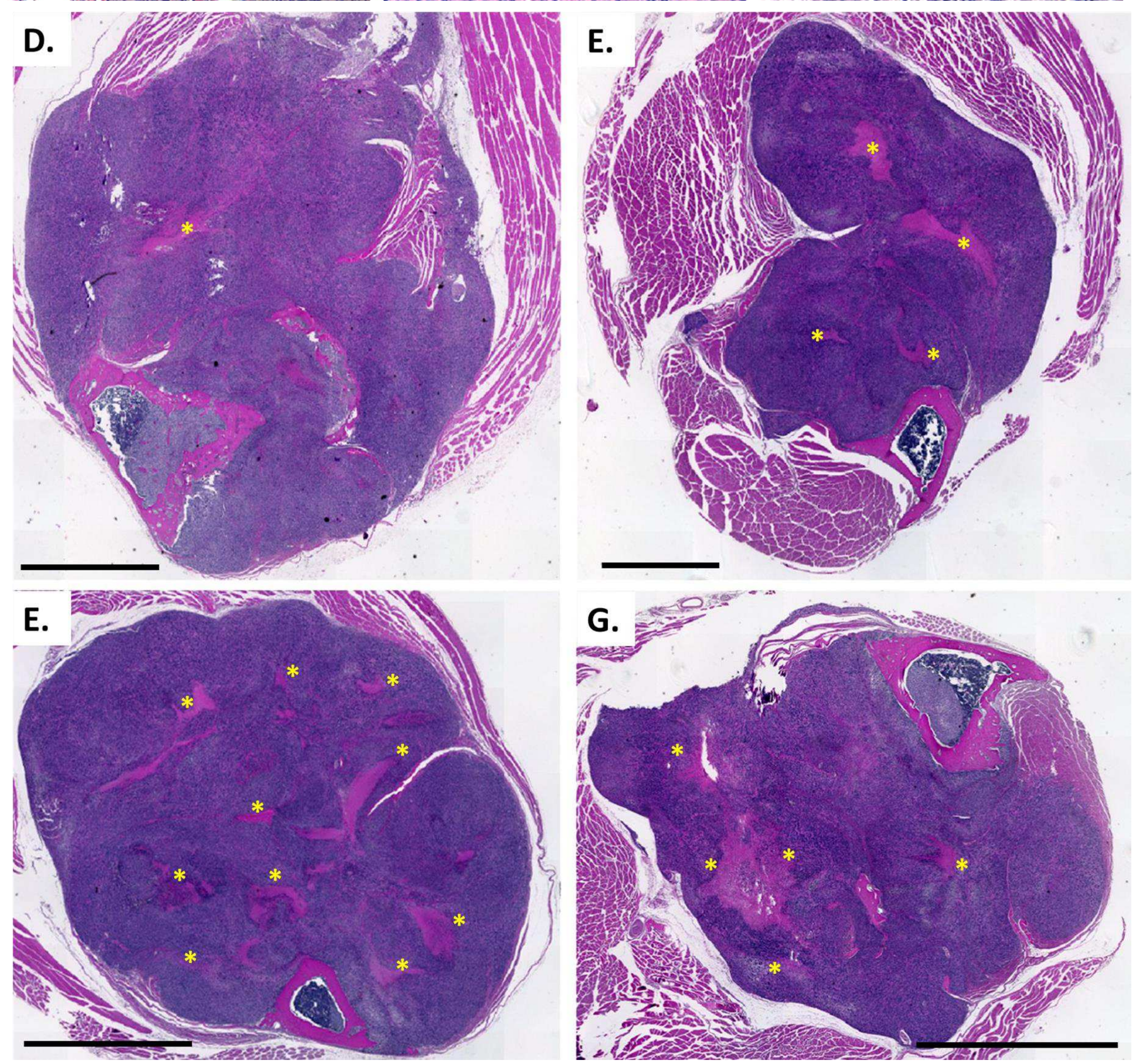


\section{Supplemental figure 4.}

a.

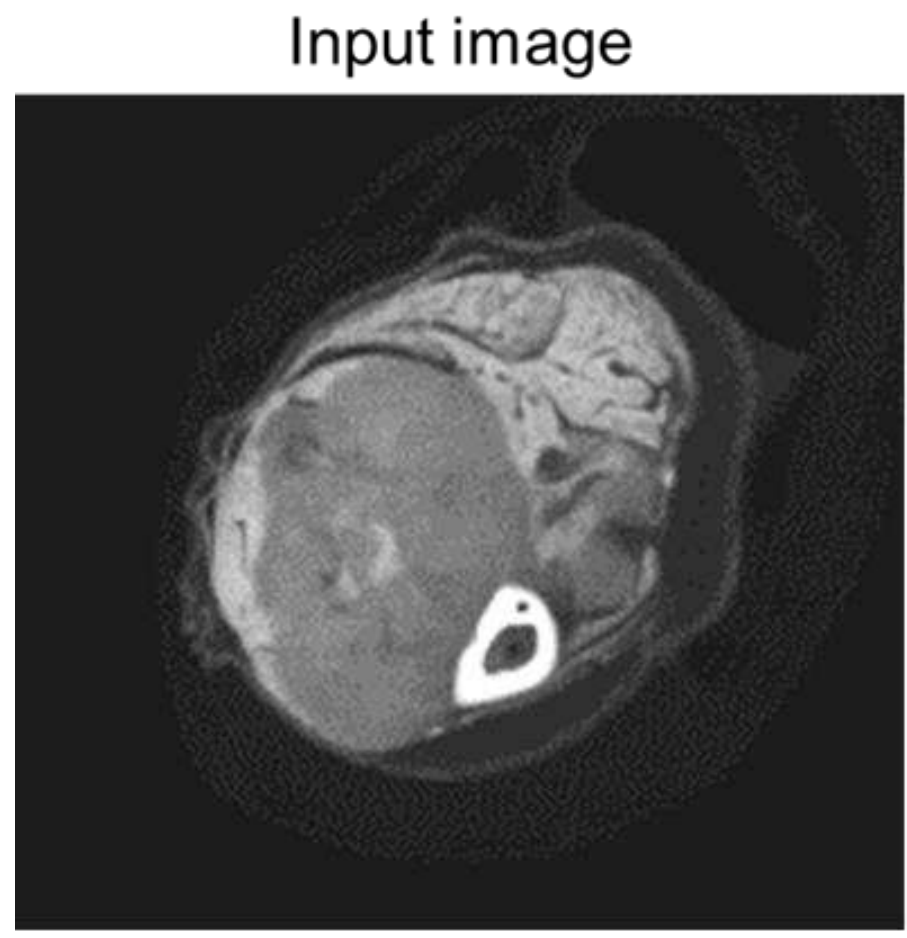

True mask

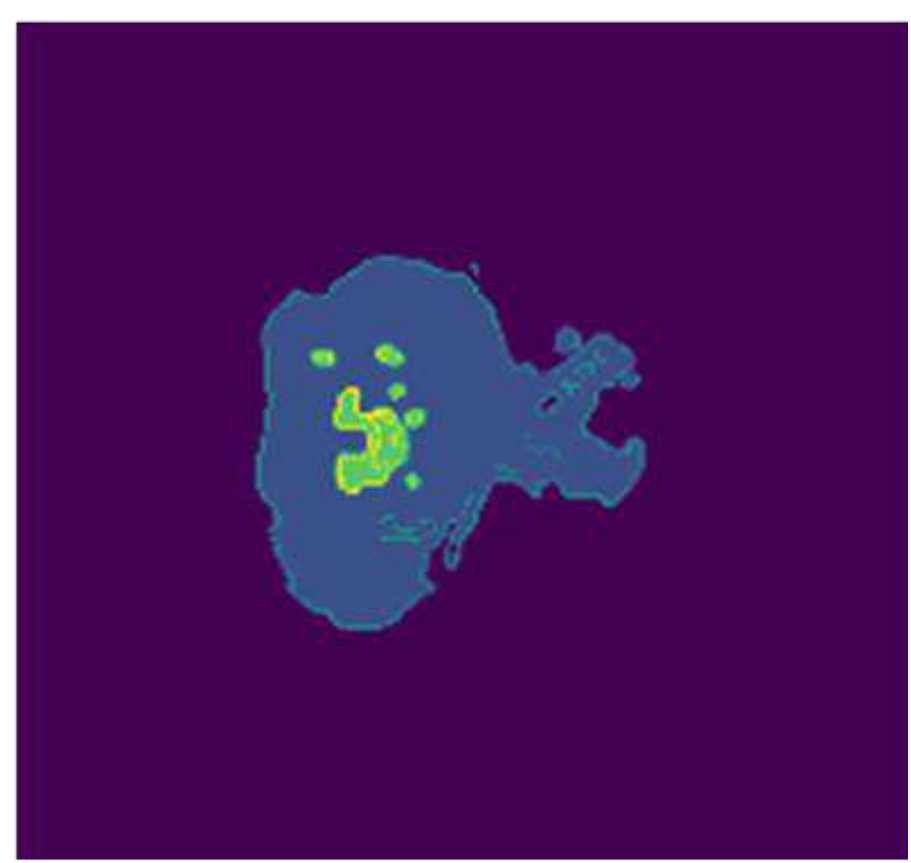

Predicted mask

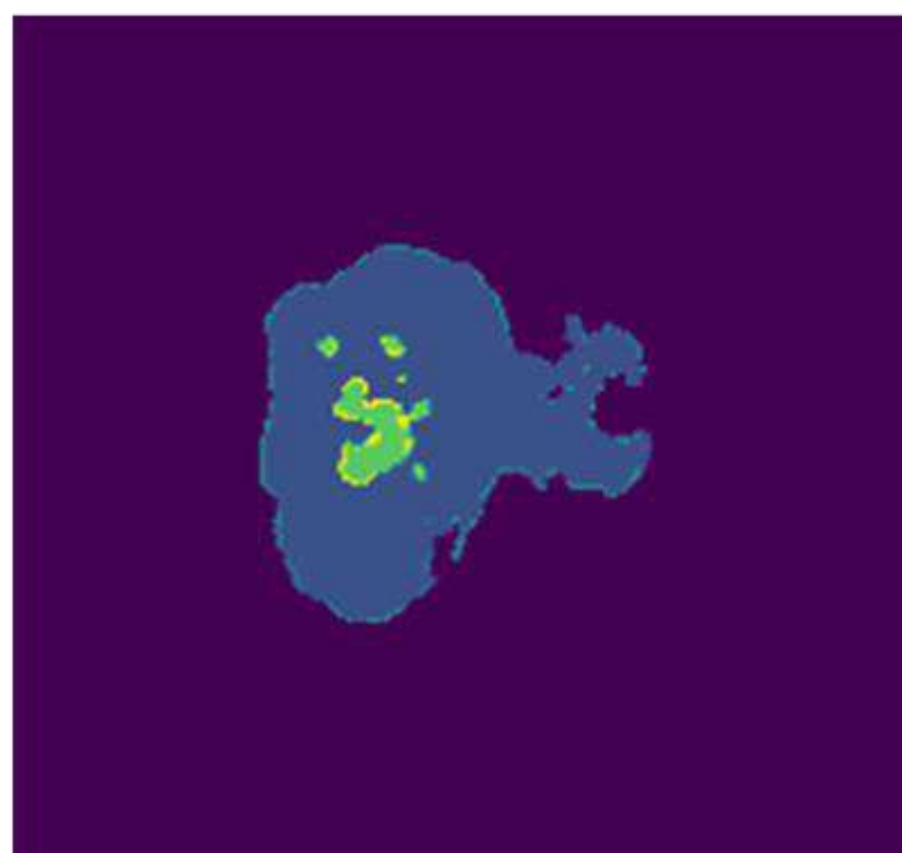

c.

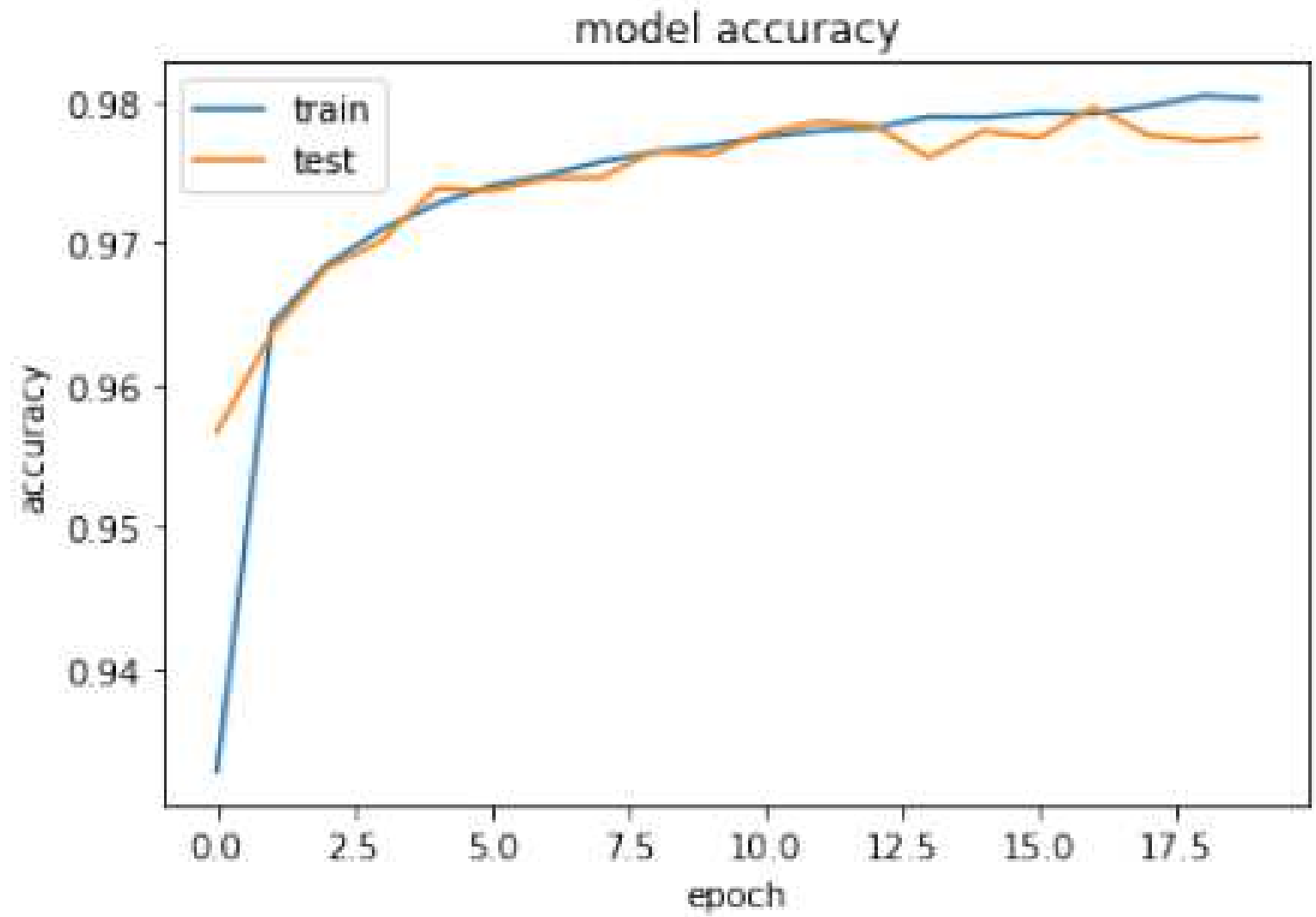

b.
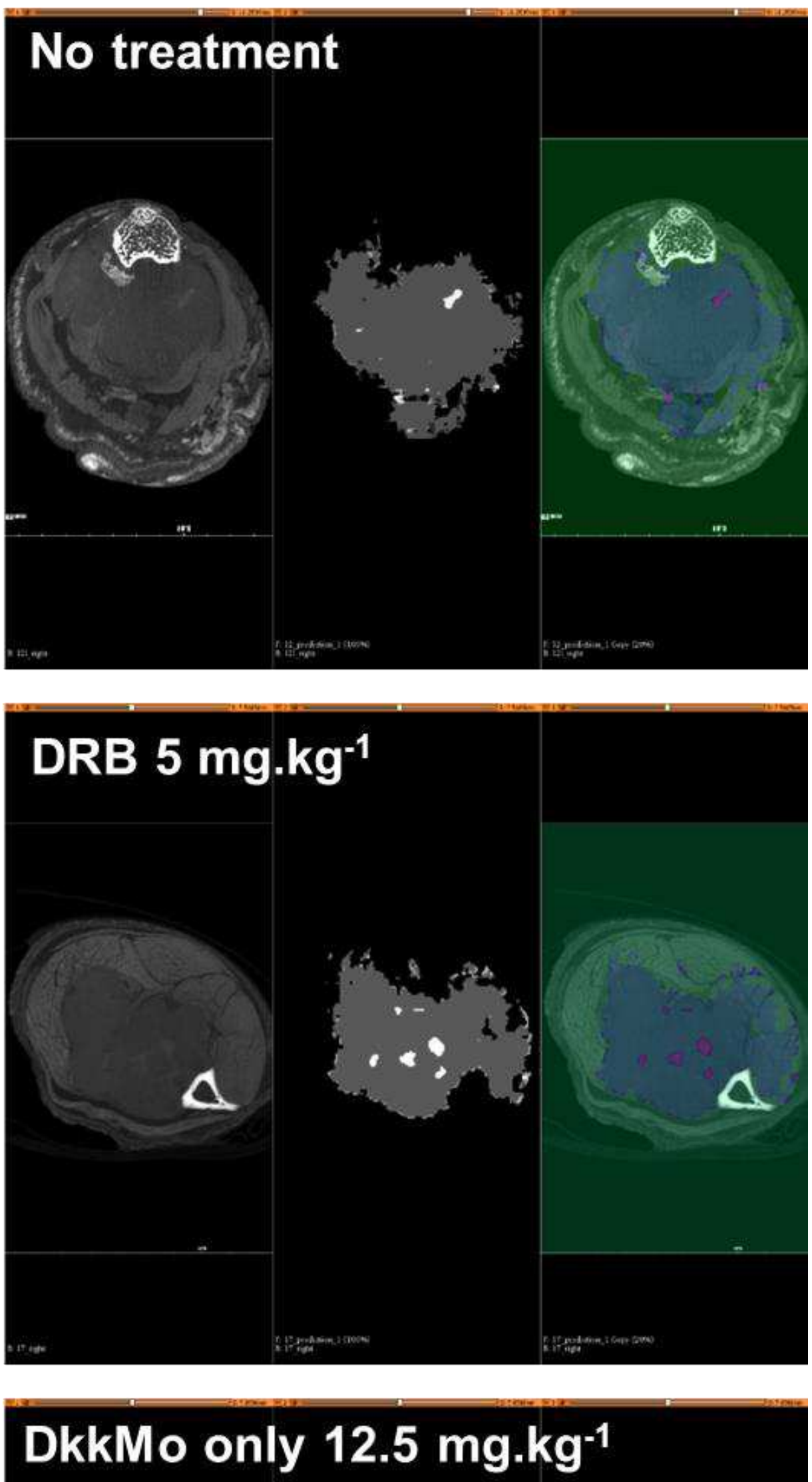

Combination

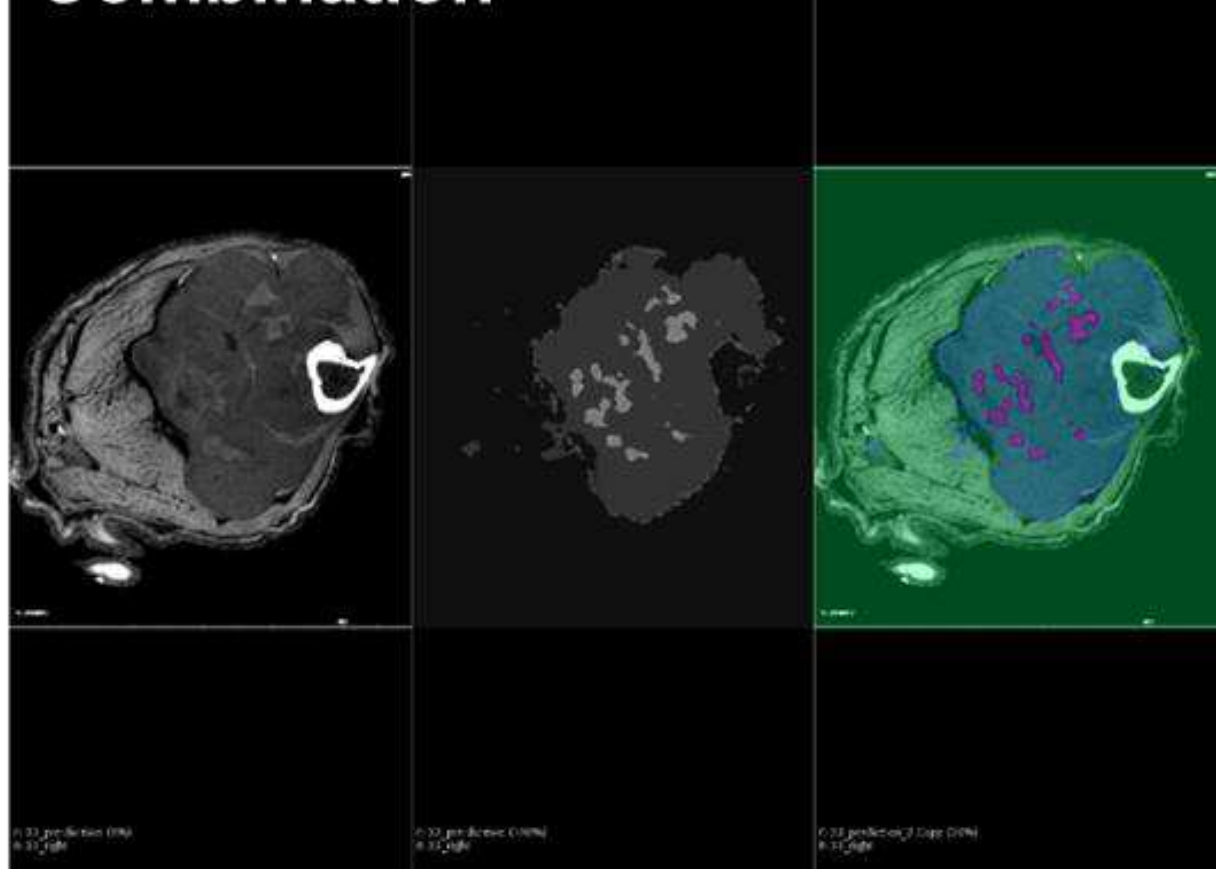



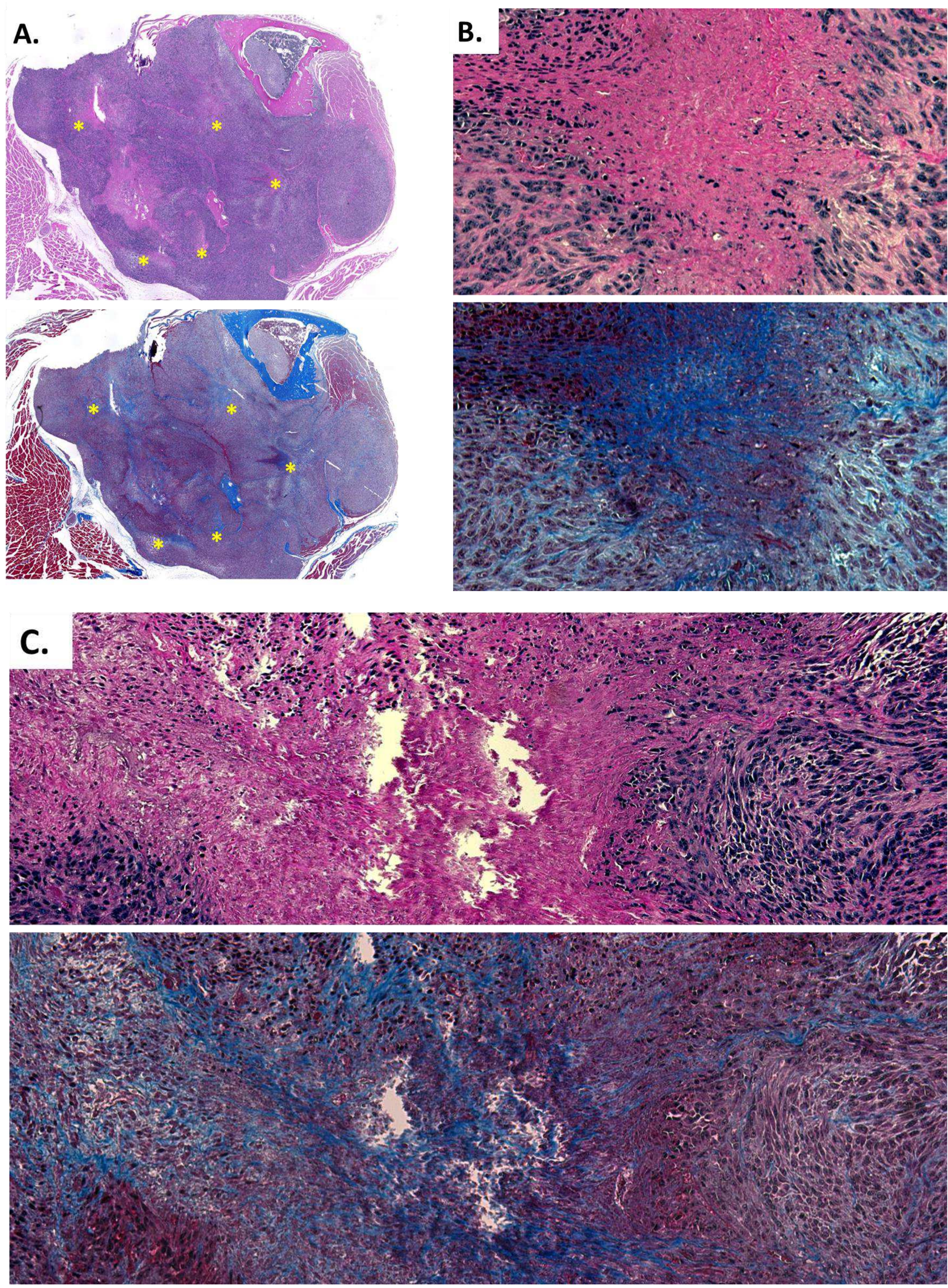
DRB versus combination

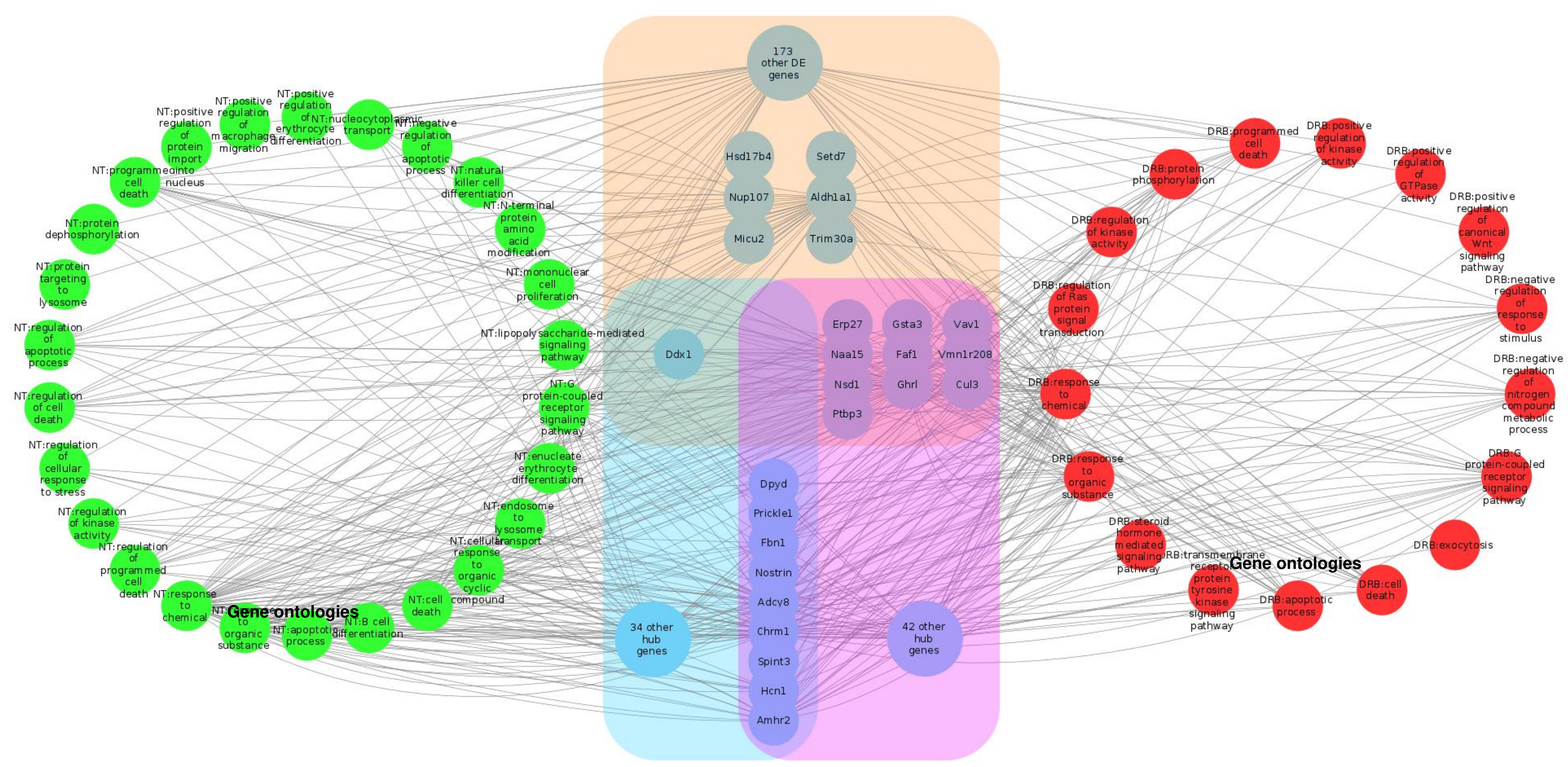




\section{Supplementary Files}

This is a list of supplementary files associated with this preprint. Click to download.

- SupplementaltablesPanetal2021.xlsx 